\title{
Section I: Oral Sessions
}

\section{ADOLESCENT \& CHILD HEALTH}

\section{1-01 Parental Monitoring and Poverty: Influences on Youth Violent Behavior in an Urban Setting}

Tracy L. Patterson; Philip J. Leaf (Johns Hopkins Bloomberg School of Public Health) Internationally, youth violence is becoming an increasing concern. This study builds upon two lines of epidemiological research on youth risk behavior. One suggests that children who are not well monitored by parents are at increased risk of engaging in risky behavior. In a second line of research, associations are made between neighborhood features and delinquency. We set out to bridge the gap between these two lines of research as they relate to youth violence. We studied a prospective longitudinal sample of 1796 public school children in Baltimore, Maryland. We examined whether high levels of parent monitoring signal reduced risk of violence (youth self-reports and juvenile court adjudication records), while high levels of family poverty increase the risk of youth violence. Secondly, we examined whether the relationship of parent monitoring with violence varies by level of neighborhood poverty. Results indicated that youth residing in high poverty neighborhoods are more likely to engage in violent behavior. However, we also found that youth who are well monitored by their parents are less likely to engage in violent behavior, regardless of the child's neighborhood poverty status. These findings can inform the development of future violence prevention programs, which should consider parenting and neighborhood factors.

\section{1-02 Health eTouch: Computerized Risk Screening in Urban Primary Care Clinics}

Deena J. Chisolm PhD; William Garnder PhD; Jack Stevens PhD; Kelly J Kelleher, MD, MPH (Columbus Children's Research Institute)

Background: Primary care is underutilized as a setting for providing substance abuse and behavioral health treatment and prevention services. Principle obstacles to service delivery include severe constraints on primary care physician (PCP) time and lack of PCP knowledge about risk assessment. Health eTouch is a computerized screening tool for behavioral health issues in primary care. Computer-assisted, self-interviewing can improve screening for adolescent behavioral health issues because the technology uses standardized questions and the screening is completed before the patient sees the clinician. It also makes it easier for youth to reveal sensitive, personal information.

Methods: Over 2,500 youth ages 11-20 were screened for substance use, depression, suicidality, and injury risk using secure, wireless, touch-screen tablets in nine urban pediatric primary care clinic waiting rooms. Clinics were randomized to either immediate or delayed delivery of results to clinicians.

Results: Patients seen in the immediate feedback clinics were more like to have their risks recognized by the clinician than those in clinics with delayed feedback. Atrisk patients in the immediate feedback group were also more likely to have 
subsequent mental health care. Patients were generally satisfied with their use experience. Level of satisfaction was not associated with race, computer experience, or payor.

Conclusions: Computer-based screening is a well accepted method to improve problem identification in busy urban primary care clinics. Giving clinicians immediate access to results from such a system improves identification and followup on behavioral health issues and can, hopefully, improve patient outcomes.

\section{1-03 Slum Communities Partner with Public and Private Health Providers for Improvement in Maternal and Child Health}

Siddharth Agarwal; Prabhat Jha; Sandeep Kumar; Partho Haldar; Rajeev Kumar (Indore Urban Health Forum)

Background: Urban poor people living in slums have insufficient access to primary health care services due to weak linkage between health care providers and slum communities.

Methods: Indore, a mid-sized Indian city has a rich culture of community level processes. The urban health program in 75 slums (150,000 population) of Indore, aims at enhancing access and utilization of health services through organization of community into functional groups. Lead CBOs (representing existing slum leaders) mobilize community, promote and build capacity of slum level CBO through, support them in counseling sessions, pursue and nurture linkages with public and private health providers and Municipal Corporation and maintain records. Slum-based CBOs (women's group promoted during the program) identify families with pregnant women and infants as well as households with special needs facilitate service utilization; track coverage; discuss and negotiate with public sector service providers for health water and sanitation services; and generate health fund to be used in emergencies. NGOs provide capacity building support to both the Lead and slum based CBOs.

Outcomes: Community organizations help identify unlisted and hidden poverty clusters in the city; they develop social and health target maps of vulnerable families in slums. Linkages have evolved with government and private sector. Regular outreach with help of government system have resulted increased access to services. Community Groups negotiate with the municipal officials to provide improved water supply thus providing advocacy voice for basic needs of underserved communities. They have steadily initiated a partnership with private doctors for monthly ante-natal check-up clinics in slums as well as follow-up maternity care.

Conclusion: Strengthened slum based CBOs serve as social capital for underserved families and generating demand and improving access to services.

\section{1-04 Partnering with English and Spanish Speaking Parents in the New York City Public Schools to Prevent Obesity and Diabetes}

Leslie Goldman, MA; Joanne De Simone Eichel, MA; Danielle Slutsker, MSW; Freya Kaufman, MA, CHES (The New York Academy of Medicine)

The New York Academy of Medicine is implementing the Partnering with English and Spanish Speaking Parents to Prevent Obesity and Diabetes program to address health disparities in urban communities where diabetes and obesity rates are disproportionately high. The program empowers parents/caregivers to play an important role in promoting the health of their children and families, through improved nutrition and increased physical activity.

By working closely with New York City public elementary schools to assess the particular needs of the school community, the program offers relevant, dynamic, and 
culturally sensitive, hands-on educational activities for parents/caregivers to build their capacity to promote their own health and that of their families. Through professional development, the program engages and builds the capacity of an interdisciplinary school-community team, teachers, and staff to implement school-wide health promotion activities using promising evidence-based obesity prevention strategies.

Seminars and workshops integrate nutrition and fitness with other relevant physical, mental and emotional health topics including stress management, asthma, and disease prevention to address issues that parents/caregivers grapple with and are often associated with obesity and diabetes. The program includes working with parent coordinators and leaders, coordination with other school-based health initiatives, technical assistance, and evaluation.

\section{1-05 Gender Differences in the Relationship between Dating Violence Perpetration and Relational Aggression among Urban Adolescents}

Jessica Roberts Williams, PhD(c), MPH, RN; Joan Kub, PhD, RN; Haera Han, PhD, RN; Jacquelyn C. Campbell, PhD, RN, FAAN (Johns Hopkins University School of Nursing)

The purpose of this presentation is to examine gender differences in the relationship between relational aggression and dating violence perpetration among 7 th grade urban African American adolescents. Given that dating violence and relational aggression are largely understudied among urban, minority youth, this presentation will improve our understanding of these behaviors in this population. In conjunction with a CDC funded dating violence intervention program, self-report questionnaires were administered to 194 seventh grade students attending four urban middle schools during Spring 2005. Descriptive statistics were conducted to determine the prevalence of dating violence, relational aggression and exposure to personal, family and community violence in this sample. Overall, the levels of violence in the lives of these adolescents were high, attesting to the high rates of violence often found in urban communities. Hierarchical logistic regression was used to examine the independent effects of relational aggression on dating violence controlling for demographic variables and exposure to personal, family, and community violence. Findings indicate that relational aggression and exposure to community violence are important predictors of dating violence among girls, but not boys. For boys, age and exposure to family violence emerged as important predictors. Implications for preventative practices and directions for future research will be discussed.

\section{1-06 Shovels, Dirt, Seeds and Kids: The Growing of Healthier Youth through Urban Gardening}

Tammy Thomas, MSW,MPH (University of Pittsburgh, Graduate School of Public Health)

Urban gardening has developed as a community-based health intervention in recent years and, most extensively, has been used with youth. Numerous programs, in schools and community-based settings, are helping inner-city youth create change in their neighborhoods, while at the same time developing skills necessary for active participation in their community. Focus for many of the programs has been on the environmental factors impacting health, including academic achievement, physical activity and nutrition, and life skills.

The recent scholarly literature, while small, shows promising results when gardening is used for youth development. However, there is a need for addition large-scale research to determine the specific impacts of gardening programs on 
youth. This presentation will provide a thorough review of the current literature, a critical and necessary step for the development of future research. The review was conducted using Psychinfo, PubMed, and ERIC and key words gardening and youth and school gardening. Additionally, references from articles on gardening were reviewed to glean additional sources. The findings from established research on urban gardening with youth will be presented and best practice suggestions for both those working with urban youth and for future research will be provided.

\section{1-07 A Comparative Analysis of the Health Expenditure of Different Socio-Economic Groups in Delhi and its Implications for Policy Makers}

Surabhi Batra; Akshat Katyayan; Jugal Kishore (Maulana Azad Medical College)

Ever-Increasing consumer expectations, awareness, demographic ageing and continuing market liberalization coupled with financial constraints in the last decade has placed high demands on the health system. In order to efficiently use the resources allocated to the health sector the policy making bodies need to have a better understanding of the health seeking behavior of the population. Methods: The study was carried out in Delhi (India), over a period of 6 months from January 05 to June 05. A total of 200 families, including 50 from slums and 50 from rehabilitation colonies, were selected using stratified random sampling after dividing the state into 9 districts. They were asked to fill a preformed questionnaire, about the household expenditure on health. After the first visit subsequent visits were made at monthly intervals for a period of 6 months with a variability of 12 days and the questionnaire was refilled. The average of 6 months was calculated and the mean data was analyzed using appropriate statistical tests. Results: It has also been shown by the study that the private clinics and hospitals get majority of their patients from posh and middle class colonies. Compared to the above, Slums and rehabilitation colonies spend a very little on private health setup. While posh and middle class colonies show a considerable spending on over the counter medicines, Rehabilitation colonies.

\section{1-08 Developing an Evaluation of Visual Voices: An Art-Based Initiative to Address Injury Related Issues among Urban Youth}

Jessica G. Burke, Michael Yonas, Andrea Gielen (University of Pittsburgh)

Visual Voices TM is an arts-based program designed to bring youth together within a common artistic venue to address issues concerning their lives, communities, and futures. The three principle mediums of expression used during Visual Voices TM are painting, drawing and writing which are collaged to create one common public exhibition. Founded in 1993, the Visual Voices TM project has worked in eight cities in the US and addressed collaboratively issues such as youth violence, dating violence, and other individual and group inspired topics. Although Visual Voices TM has received positive reviews from participants and previous funding agencies, no formal evaluation has been conducted of the project. With the goal of developing evaluation materials, youth between the ages of 8 and 14 were recruited from a local community-based organization in Baltimore City. The youth participated in four two-hour project sessions and two subsequent focus group discussions conducted to explore their experience with the project. Questions asked referred to participant perceptions of the overall process, information learned, favorite and least favorite elements, and what could be done to improve the project. With permission, focus group discussions were tape-recorded, transcribed verbatim, and analyzed using qualitative thematic data analysis. This interactive presentation will provide an overview and examples from the Visual Voices TM sessions, present findings from the focus groups, and describe the developed process and impact evaluation materials. 


\section{1-09 Childhood, Work and Health: The Bolsa-Escola Case of the City of Belo Horizonte (Brazil)}

Michelle dos Santos Diniz; Ada ývila Assunýýo; Mery Natali Silva Abreu; Waleska Teixeira Caiaffa (Universidade Federal de Minas Gerais - Faculdade de Medicina) INTRODUCTION: Child labor is increasing. Worldwide, two hundred and fifty million children and adolescents aged 5 to 14 are employed in the workforce. In Brazil, government data of 2001 shows that the child labor force (5 to 17-years-old), is comprised of over five million children, accounting for $12 \%$ of the children in this age group. The School Welfare Program Programa Bolsa Escola implemented in 1997 in the city of Belo Horizonte, Brazil, aimed at improving school enrollment and attendance in public schools of children and adolescents ( 6 to 15 years of age) from poor families. At the moment, the program involves 11,627 families.

OBJECTIVE: To study socio-demographic characteristics (profile) of the families under the school welfare program (nuclear family, number of family members and the educational level of the mother) in which child labor cases were detected.

METHODS: This is a descriptive study. From the database of families under the school welfare program in which child labor cases were detected in 2003/2004 (1,827 families), we identified those still in the program in September 2006 and studied their family profile.

RESULTS: Of the 1,827 families, 1,294 (71\%) were still in the program in September 2006, of which 248 families (19\%) had up to four members and 1,046 $(81 \%)$ had five or more members. The nuclear family was biparental in 556 families $(43 \%)$ and monoparental in 738 families $(57 \%)$. The mother's education was low (0-3 years) in most families $(677,52 \%), 4$ to 7 years in 523 families $(40 \%), 8$ to 10 years in 61 families $(5 \%)$ and more than 10 years in $11(1 \%)$ families.

CONCLUSION: The socio-demographic characteristics of families may play an important role in child laborýs contributive factors. This subject will be the object of study of the next phases of our research.

\section{1-10 Maternal Education or Household Wealth: Which is the Best Predictor of Child Malnutrition in Urban Sub-Saharan Africa?}

Jean Christophe Fotso (The African Population and Health Research Centre (APHRC))

Though a continually growing body of research suggests the prominence of maternal education as predictor of child health and survival in developing countries, the extent to which the relationship merely reflects the impact of economic advantage on health and survivorship, or the peculiarity of these associations in urban areas, have not yet been fully elucidated. One of the key features of Africa is the high and rapidly growing urban poverty, which makes it plausible that in the near future most of the poor people in the region will live in urban areas. This paper examines Demographic and Health Surveys data of 23 countries in sub-Saharan Africa with the goal of 1) comparing the effects of maternal education and household wealth on urban child malnutrition, and 2) investigating the pathways between maternal schooling and household wealth as determinants of child undernutrition in urban areas. Multilevel, multivariate regression results show that household wealth tends to be more robust, and to have higher power in predicting child malnutrition, than maternal education. This finding therefore suggests that while education remains critical in improving child health in urban areas, wealth-related factors such as improved water and sanitation are also at least as important. 
01-11 Evaluation of the SAGE LIFESKILLS and GRACE Programs: Addressing Trafficking and Commercial Sexual Exploitation Among Adolescent and Young Adult Females Marcia Cohen, MCP; Mark Edberg, PhD; Stephen Gies, PhD; Norma Hotaling; Karen Bachar, PhD (Development Services Group, Inc.)

The SAGE (Standing Against Global Exploitation) Project, Inc., offers an array of services to young women who are victims of commercial sexual exploitations (CSE). The LIFESKILLS program is an out of custody program for girls 18 or younger who are CSE victims. The Girls Reaching Adulthood Through Community Empowerment (GRACE) program serves a slightly older population (18- to 24) of females. Both programs utilize a cognitive behavioral approach. The evaluation study is composed of a three-phased design: formative; outcome; and generative. The formative phase uses focus groups to identify the model and operationalize specific outcome variables. The outcome phase focuses on the success of the participants in the programs. It utilizes a repeated measures design to assess participants. The outcome measures include: level of involvement in CSE, delinquency, substance use, degree of victimization, commitment to school, attitude toward employment, PTSD symptomology, self efficacy, degree of social support, and participation in exploitative relationships. Finally, the generative phase identifies factors through narrative interviewing/analysis that may serve as salient variables for future research. This research addresses the lack of adequate information on factors related to entry, retirement and recovery from commercial sexual exploitation. Findings from the formative phase will be discussed.

\section{1-12 Neighborhod Disadvantage: Influences on Youth Violent Behavior in an Urban Setting.}

Tracy L. Patterson; Philip J. Leaf (Johns Hopkins Bloomberg School of Public Health)

Violence in school-age children is a problem in many countries. We extend prior research on neighborhood effects on youth violent behavior by utilizing youth selfreports of neighborhood disadvantage as risk for engagement in violence. The urban sample consists of predominately African-American adolescents of lower socioeconomic status who participated in a prospective longitudinal study in Baltimore, Maryland. We used logistic regression analyses to examine the associations between youth self-reports of neighborhood disadvantage in the sixth grade with self-reported violence in grades 6,7 and 8 and with official juvenile court adjudication records. Youth reports of neighborhood disadvantage were associated with youth violent outcomes regardless of how those outcomes are measured. Increasing levels of neighborhood disadvantage were positively associated with engagement in violent behavior. Being African-American was significantly associated with having a youth violent court adjudication by age 14, but was not significantly associated with self-reported violence. Youths' experiences of their neighborhoods should be taken into consideration when designing violence prevention and intervention studies. Future research in policy and practice should also address the issue of racial disparities in the juvenile justice system.

01-13 Vulnerability of Street Children to Sexually Transmitted Diseases and HIV Infection in Kathmandu, Nepal

Ram Saran Adhikari (National Resource Center, Nepal) (National Resource Center, Nepal)

Background: Areas around bus parks are a home to large number of children who runaway from home. Such migrants mostly come from Maoist affected areas. They are easily forced into substance abuse and high risk sexual behavior due to 
peer pressure from senior street children. This study is designed to examine the health problem of these children.

Methods: Kathmandu Municipality runs health posts at 5 different wards in Kathmandu. The data are based on records of the health post in 8 months period from May and December 2006. Those children visiting the health posts were asked to fill in a questionnaire and a nurse based interview was conducted.

Results: 488 children visited the health post. $27 \%$ were girls and $73 \%$ were boys. $115(23.56 \%)$ were treated for sexually transmitted illness (STI) and 30 were tested for HIV and 1 was found to be HIV positive. About $55 \%$ of children reported history of high risk sexual behavior. $45 \%$ reported poverty and employment opportunities in Kathmandu as the pull factor to motivate them to run away from home.

Conclusion: These children require sex education to reduce the risk of infection, health care and social support services and rehabilitation efforts in order to protect their lives.

\section{1-14 Sex Education, Knowledge, Attitudes and Behavior of Adolescents Towards HIV/ AIDS in Ghana}

\section{Akwasi Kumi-Kyereme}

Alex Ezeh (University of Cape Coast)

The aim of this paper is to examine differences in HIV/AIDS knowledge, attitudes and behavior of adolescents who have ever attended sex education classes and those who have not. This paper draws from 2004 nationally representative household-based survey of 4548 adolescents aged 12-19 years in Ghana. Logistic regression models are used to examine associations of knowledge, attitudes and behavior related to HIV/ AIDS and attendance at sex education classes. The results show that majority of adolescents have accurate knowledge about the modes of HIV/AIDS transmission even though misconceptions are common. Adolescents generally have negative attitudes towards people living with AIDS. Attendance at sex education classes significantly affects adolescents' misconceptions and attitudes but not their behavior. To the extent that knowledge and attitudes can inform future behavior, we could expect attendance at sex education classes to ultimately influence adolescent behavior.

\section{1-15 Selected Experiential Predictors of Breastfeeding Initiation among Wic-Eligible Mothers at an Urban Private Hospital in 2000, 2002, 2004, and 2005}

Dina B. Passman, MPH (School of Public Health and Health Sciences, The George Washington University); Jennifer E. Marsh, JD, PhD, MSN (Center VI, Children's Research Institute, Children's National Medical Center); Jennifer A.F. Tender, MD (General Pediatrics, (The George Washington University, School of Public Health and Health Services)

Context: We examined the initiation of breastfeeding in low-income AfricanAmerican women giving birth at a private hospital in the District of Columbia in order to better understand the rates of breastfeeding immediately post-partum in this population. Several studies have found a relationship between breastfeeding and race, suggesting that African-American women are less likely than women of other races to initiate breastfeeding their newborns. However, such a conclusion requires a greater understanding of the specific processes linking African-American race and breastfeeding initiation, particularly birth experience.

Method: We conducted descriptive and multiple regression analyses of data from 511 mostly African-American $(n=441)$ mother-infant pairs to test the 
relationship between medical elements of birth experience (type of delivery, medication use, and time/day of delivery) and post-partum breastfeeding initiation in the hospital.

Results: We found no statistically significant associations between elements of birth experience and initiation of breastfeeding during the post-partum hospitalization.

Conclusions: We found no evidence that birth experience is associated with inhospital breastfeeding initiation in African-American women. Our results suggest that interventions focusing on birth experience to increase breastfeeding initiation rates may therefore lack efficacy. As a result, policy makers should rethink current breastfeeding policy and continue to focus on social factors affecting breastfeeding initiation and longevity, in particular among low-income, urban African-American women.

\section{1-16 Involvement in High-Risk Behaviors among Youth who Live in Disadvantaged Urban Communities Relative to the U.S. Average}

Monica H. Swahn, PhD; Robert M. Bossarte, PhD (Centers for Disease Control and Prevention)

Objective: To define, quantify and compare involvement in high-risk behaviors by youth in disadvantaged urban communities relative to the U.S. average.

Method: Data from the "Youth Violence Survey" conducted in 2004 and administered to students $(\mathrm{N}=4,131)$ in a disadvantaged school district were compared to the 2003 national Youth Risk Behavior Survey and the Add Health Study conducted in 1995/96. The differences in prevalence of risk and protective factors among 9 th grade students from the three studies were assessed using Chi Square tests.

Results: Youth living in disadvantaged urban areas were significantly more likely than their peers across the country to report vandalism, theft, violence and drug selling. Youth in disadvantaged urban areas also reported significantly less support from their homes and schools, and less monitoring by their parents. Moreover, youth in disadvantaged urban areas were significantly less likely to binge drink or initiate alcohol use prior to age 13 than youth across the U.S.

Conclusions: Youth in disadvantaged urban communities tend to report significantly higher prevalence of some, but not all, risky behaviors than average US youth. These findings should be considered when defining and planning new research and programming targeting youth at high-risk for health-compromising behaviors.

\section{1-17 Policy to Promote Bicycle Commuting}

Catherine Harbour, MPH (Doctoral student in health communication Department of Health, Behavior, \& Society, Bloomberg School of Public Health, Johns Hopkins University); Jamie Bridges, MA (Transportation Planner Baltimore Metropolitan Council) (Johns Hopkins Bloomberg School of Public Health)

Bicycle commuting is a sustainable and health-promoting form of transportation. Bicycle commuting provides regular and moderate physical activity, is affordable, and does not pollute. Compared to motorized transport, bicycle commuting costs less and requires less expenditure for road maintenance and parking infrastructure. Public policy at the local, regional, and national levels and workplace policy can encourage or discourage bicycle commuting. Public policies related to bicycle access routes are often coupled with pedestrian access routes, although bicycling allows commuters to cover greater distances and more comfortably carry things than does walking. The presenters will review some examples of policy that can promote commuting by bicycle, such as the Safe Routes to Schools legislation, university bike plans, and worksite bicycle policies. The presenters will profile public policy and workplace policy related to bicycle 
commuting in Baltimore and offer suggestions for improving policy so as to increase bicycle commuting. Monitoring and evaluation of the impact of these policies has been inconsistent, and the presenters will suggest how better monitoring and evaluation could more accurately quantify the impact of policy to promote bike commuting.

\section{BEST PRACTICES IN MEETING URBAN HEALTH CHALLENGES}

\section{2-01 Susceptibility and Vulnerability of Urban Population to HIV/AIDS: A Case Study of Kathmandu, Nepal}

Sabin Maharjan (National Health Care Pvt. Ltd)

Objectives: HIV prevalence rate among sex workers is 17.3 percent and 49.2 percent among intravenous drug users (IDUs) in Kathmandu. This case study of Kathmandu is part of a multifactorial assessment designed to analyze various factors directly involved for confronting HIV/AIDS.

Methods: In depth interviews with 20 commercial sex workers, 25 truck drivers and 30 sex workers who had returned from Indian brothels was conducted.

Results: Findings show that 30 percent of the sex workers were unaware about HIV infection when they got involved in the business. 47 percent of high way truckers are involved in unsafe sex. Condom use is less than 5 percent. 40 percent of sex workers who returned from India are found to be HIV positive and 18 percent are infected with AIDS. Basic human rights are denied to them and they report of being victims of discrimination in the society. This study is an exemplary effort to reach out to the HIV infected subjects and high risk groups to understand the root cause of HIV/AIDS.

Conclusion: HIV/AIDS related educational programs among clients visiting massage centers and high way truckers are strongly recommended. Education for improving knowledge and condom use and community based interventions should be considered for prevention.

\section{2-02 Implementation of a Rapid HIV Screening Program in a Large County Jail}

Dana K. Rice, MS; Sandra Peppers; Timothy Barth, MD; Keith Dlugokinski, PhD (Wayne County Jail)

The prevalence of HIV/AIDS among inmate populations is five times higher than that in the general U.S. population. However, very few jails provide routine opt-out HIV testing upon entry. Therefore, current prevalence estimates among jail inmates underestimate the real occurrence in correctional settings. The purpose of this pilot project is to develop an opt-out HIV screening program in the Wayne County Jail, utilizing novel rapid screening technology, and coordinated inter-departmental and inter-agency procedures for reporting, treating, and follow-up care. An on-site jail healthcare provider will offer a sample of high-risk, non-violent sentenced offenders, a rapid HIV screening exam, on an opt-out basis, as a component of the initial health appraisal. Results and post-test counseling will be provided during that initial point of contact. Reporting of tests, results and counseling will occur through a collaborative effort between the jail, the local health department and the state health department. This program creates a basis for comparison of current, risk-based, selfinitiated requests for HIV testing in correctional facilities to the recent CDC recommendations for opt-out consent procedures prior to HIV testing. This innovative program may provide a viable public health practice standard for conducting HIV screenings in large jail settings. 


\section{2-03 Nueva Vida's Pshycho-eduactional Support Group for Latinas with Cancer}

Adriana Kaufman, MA; Lidia Carnota, MD; Lydia Buki, PhD (Nueva Vida)

Purpose: The purpose of Nueva Vida's Survivorship Support Program is to offer comprehensive, caring and culturally expert mental health support to Latinas throughout the continuum of breast cancer survivorship including diagnosis, treatment and recovery. Program includes: Intake interview to asses women's needs; general and stress reduction support groups; therapeutic group for women in treatment; individual counseling for women in crisis or with advance disease; peer support for women recently diagnosed or in treatment.

Methods: The General Support Group gathers women at all stages in the cancer experience and their families. They come together to share, learn, give and receive support. The goal is to offer psychosocial support to women at all stages post diagnosis through psycho-educational activities. The General Support Group is designed to empower participants and promote their adjustment towards a healthy life as a survivor. Each monthly meeting addresses a different topic, some of them are: nutrition, communications, self-image, fears and the effect of cancer on the family. The co-facilitators are two Latina mental health professionals; -one of them is a survivor-. They promote an environment where participants may express themselves, ask questions, share experiences, feel supported and support other members. The model of the General Support Group has evolved over the years. Its content and structure were modified and adapted to meet the special needs of Latina survivors, so they can feel comfortable and trust the program. Participant's experience and program quality is assessed with the administration of a comprehensive, anonymous end-of year survey. Program use is measured with the number of participants and retention rates in program activities.

Results: During 2006, 90 people participated in Nueva Vida's psychoeducational Support Group with an average of 15 in each session.

\section{2-04 Public Health Management of Chronic Heroin/Cocaine Addicts with HCV and HIV in Amsterdam}

Giel van Brussel; Marjolein Verstappen; Maria Prins; Karen Lindenburg; Christine Weegink (GGD Amsterdam)

Amsterdam has a large population of heroin/cocaine addicts. The harm reduction model, using an epidemiological approach, low threshold Methadone Maintenance Treatment (MMT) and Needle Exchange Programs (NEP) has been relatively successful in controlling the spread of IV drug use, e.g. heroin addiction and concomitant infections. Adequate specialist medical treatment of addicts with HCV and HIV conditions is necessary.

Most patients are motivated for treatment but often are not compliant with necessary hospital visits/examinations. Appointments are frequently missed. As a consequence marginalized drug addicts in reality have no access to theoretically available treatment for HIV/HCV. To improve this situation hospital specialists for HIV and HCV are located near the Public Health low threshold MMT/NEP programs in the form of an outpatient clinic that combines medical specialist care for HCV and HIV with public health addiction care. With this method also drug patients with problematic behaviour and an active addiction can be motivated for treatment with good results in the form of full compliance.

The cooperation will be described, including the specification of each contributing partner, including the health insurance. The way the cooperation is developed can be a model for other cities where problematic heroin addicted populations exist. 


\section{2-05 SMILES}

Ms. Veron L. Ash, RN (St. Michael's Hospital)

The Stroke Management In Lifestyle Education Seminar (SMILES) is a best practice education and awareness program designed to meet the unique needs of each participant related to a global health challenge. This four (4) hours seminar raises the level of awareness about stroke and transient ischemic attack (TIA) for participants using an interdisciplinary approach. Although the program specifically targets stroke and TIA clients seen in the Emergency, Secondary Prevention (TIA), and Stroke Clinics at St. Michael's Hospital in Toronto, friends and family members are encouraged to attend. The SMILES Program unites TIA and stroke survivors with expert health care providers who impart knowledge about the effects and impact of stroke, support risk factor reduction, promote best practice stroke care, and facilitate access to community support and resources in one seminar. Evaluations consistently revealed a positive impacted on participants' ability to navigate the health care continuum and improve their quality of life.

\section{2-06 University Initiative Encourages Urban Health Research}

John A. Steward, MPH; and Michael P. Eriksen, ScD (Georgia State University) As a result of a university-wide strategic review, Georgia State University created an Urban Health initiative that will be phased in over 4 years with more than $\$ 2.3$ million of continuing State-supported appropriations annually. The investment has resulted in the recruitment of 16 Urban Health faculty positions in departments across the University.

The Institute of Public Health of Georgia State was designated to manage and anchor the Urban Health emphasis. A Partnership for Urban Health has been formed to organize and to provide a framework for research efforts by the 16 new faculty as well as existing faculty with Urban Health research interests. The purpose of the Partnership is to foster interdisciplinary collaboration, to seek a better understanding of the factors that affect the health and well-being of urban dwellers, especially those populations with a disproportionate share of illness and disability, and to improve urban health status. Four priority areas- HIV/AIDS and infectious diseases, chronic diseases and aging, injury and violence, and substance abuse and mental health- are addressed.

Currently, 61 faculty (new and existing) have affiliated with the Urban Health initiative. Because of the initiative, outside grants have increased, and researchers are collaborating on new Urban Health-related research projects.

\section{2-07 Does It Matter if Mothers or Fathers Ethnicity is Used in Children Studies? Ethnicity and Umbilical Cord Blood Lead Level in Pakistan}

Naveed Zafar Janjua (Department of Community Health Sciences, Aga Khan University, Karachi Pakistan)(Department of Epidemiology, School of Public Health University of Alabama at Birmingham) (Aga Khan University/University of Alabama at Birmingham)

Background: Karachi is a cosmopolitan city of 12 million people where all five major ethnic groups of Pakistan live together. They originated from various parts of country and have specific cultural practices and beliefs that affect their health behavior and status.

Objectives: To assess umbilical cord lead level across maternal and paternal ethnicity. To assess concordance between ethnicity of mother and father and compare umbilical cord lead levels of infant born to ethnic discordant and concordant parents. 
Methods: We conducted a cross-sectional study of infants and mothers at the obstetric units of two tertiary care hospitals in Karachi. We obtained information from 540 mothers about lead exposures and collected umbilical cord blood for lead levels. We compare mean lead level by maternal and parental ethnicity. We created a dummy variable for ethnic discordance. We compared log transformed umbilical cord lead level by ethnic discordance using t-test and by ethnicity using ANOVA.

Results: Only 4\% (23/540) of the newborn had ethnic discordant parents. There was no significant difference in umbilical blood lead levels between ethnic concordant and discordant parents infants $(\mathrm{t}=0.18, \mathrm{P}=0.8561)$. Umbilical cord lead levels differed across ethnic groups. Infants born to Balochi mothers (11.8) had significantly higher umbilical blood lead level than other ethic groups (Mohajir $=9.5$, Sindhi $=8.1$, Punjabi=9.7, Pukhtoon=9.0, $\mathrm{F}=2.74, \mathrm{P}=0.02$ ).

Conclusion: Very few people marry across ethnic groups in Karachi Pakistan. Risk behaviors particular to ethnic group get clustered like lead levels across ethnic groups differed. Hence, ethnicity specific behavioral interventions are important.

\section{2-08 An Integrated Pest Management Demonstration Project in Boston Public Housing: Evaluation Methods and Results}

Deirdre Pierotti;Patricia Hynes (Boston University School of Public Health)

The Healthy Pest-Free Housing Initiative, a community-university-city collaborative, is implementing an Integrated Pest Management (IPM) demonstration project and evaluating the effectiveness of the IPM program in reducing cockroach infestation in Boston public housing. Cockroach infestation is widespread in public housing, and associated with triggering asthma symptoms and attacks in sensitive individuals. Our earlier studies have found an asthma prevalence of $25-30 \%$ among children in Boston public housing, elevated and widespread concentrations of cockroach allergen, and resident use of multiple legal and illegal pesticides. IPM is a multi-pronged approach that includes establishing baseline pest conditions, sanitation, housing repair, resident education, use of less toxic pesticides, and recordkeeping.

The evaluation has 5 key hypotheses regarding the impact of the IPM demonstration project: 1.)The number of infested units will be significantly reduced; 2.) The work orders submitted by residents for pest infestation will be significantly reduced; 3.) The number of requests for transfers based on asthma and pest infestation will be significantly reduced; 4.) The results of annual standardized housing inspection surveys will show significantly improved scores on infestation conditions; 5.) Resident self-reported health and level of stress will change for the better. We will present the elements of the IPM program and model of evaluation, and report on the evaluation results from year 1 of the IPM program in 5 housing developments.

\section{2-09 Collaborating On-line to End Homelessness in Toronto}

Michael J.T. Shapcott (The Wellesley Institute)

After eight months of research and consultation on housing and homelessness with homeless people, housing professionals, policy experts and government officials, the Wellesley Institute launched its Blueprint to End Homelessness in Toronto with a traditional media launch. At the same time, we launched into the blogosphere with community partners and reached thousands of professionals, service providers and community organizations. Soon after, our housing and homelessness wiki was launched and, in its first two weeks, drew more than 1,000 visits as individuals and organizations worked to take the Blueprint to a deeper level. The wiki allowed 
information to be shared, and helped researchers, advocates, government officials and community partners build better housing policy. Sharing research on-line is important, and moving the work up a notch to collaborate electronically in research and policy adds a powerful new dimension. At the same time, Wellesley has created other virtual tools and forums to support local and far-flung networks of community-based researchers. The Wellesley Institute is a Toronto-based non-profit research and policy institute that works to advance urban heath and health equity through a unique three-pronged combination of community-based research, capacity-building and public policy research and action.

02-10 Increased Family Income Enhances Household Food Security: Results of a Pilot Project in Urban Slums of Dhaka, Bangladesh

Golam Mothabbir Miah; Gudrun Stallkamp; Asfia Azim; Mohammed Ashequr Rahman; Shamsul Huda Patwary Nazrul Islam Bulbul; Syed Izaz Rasul (Concern Worldwide Bangladesh)

Background: Household $(\mathrm{HH})$ food insecurity is a key factor contributing to malnutrition among extremely poor $\mathrm{HH}$ in slums of Dhaka, Bangladesh. Concern Worldwide Bangladesh implemented a one-year pilot project, embedded within a five-year urban nutrition project. In the pilot project, partner organizations facilitated small-scale businesses through micro-credits and training.

Objective: To assess the extent to which an increased $\mathrm{HH}$ income improves $\mathrm{HH}$ food security of project families.

Method: Data were collected from $137 \mathrm{HH}$ at baseline (BL, Sep 2005) and 117 $\mathrm{HH}$ at endline (EL, Oct-Dec 2006). Interviews were conducted using pre-coded questionnaires for household and child $(<5 \mathrm{y})$ level information on income, expenditure, food consumption; anthropometric measurements were taken.

Results: At BL, none of the $\mathrm{HH}$ had an income above BDT 3000, the extreme poverty threshold in Bangladesh. At EL, $89 \%$ of $\mathrm{HH}$ had a monthly income above BDT 3000 , and $20 \%$ of the $\mathrm{HH}$ had an income above BDT 5000 (poverty threshold). The monthly median $\mathrm{HH}$ food expenditure increased from BDT 1,600 to 2,710. The percentage of food expenditure per total monthly expenditure fell slightly from $65.3 \%$ to $61.6 \%$. The mean dietary diversity score increased from BL to EL both in young children (2.2 to 3.7) and at household level (4.1 to 5.7). Anthropometric and further food consumption results will be available at the time of the conference.

Conclusion: The increased family income improved household food security and diversified food consumption. This also enabled households to purchase other necessary goods. After a one-year period, households were able to purchase a more diverse diet for young children and household members in general.

Recommendations: Community-based nutrition programmes in urban areas with an extreme poor population should include such a food security component to improve access to food.

\section{2-11 Unaccompanied Minors: Invisible Victims of an International Crisis}

Minal Giri, MD, FAAP (Melrose Park Pediatrics)

Unaccompanied minors (also known as separated children) account for an everincreasing number of migrants entering the US. Many are victims of ethnic/political persecution, war, abandonment, abuse, and sexual/commercial exploitation.

They are often abducted, trafficked, and smuggled as victims of an inconceivable network of illegal commercial enterprises. Often, they do not understand why they are here nor are they able to tell their stories, either due to language barriers or 
deficits in maturity and comprehension. These children vary in age and come from different parts of the world: Latin America, India, China, Eastern Europe, and Africa. The one thing they have in common is their extreme vulnerability.

In 2005, the Department of Homeland Security apprehended and detained 7,787 such minors; an increase of $25 \%$ from the previous year. In US immigration courts, unaccompanied minors are treated like adults with no special consideration for needs as children. Once apprehended, they are placed in shelters and detention facilities throughout the country for indeterminate lengths of time. Although freedom from arbitrary detention is considered a fundamental human right, unaccompanied minors are routinely held in custody without having committed any crime.

Unaccompanied minors face innumerable challenges: barriers in communication (linguistic, cultural, developmental) and lack of child-focused protection and social support structures in an alien environment.

Issues of concern regarding the treatment of unaccompanied minors include:

Limited access to appropriate pediatric care (mental health in particular)

Punitive detention conditions

Violations of child rights: no right to legal counsel

Lack of professionals trained to do expert evaluations to support their asylum/ immigration cases.

This talk is designed to raise awareness and promote advocacy to protect the particular vulnerabilities of unaccompanied immigrant children.

\section{PUBLIC HEALTH INFORMATICS IN CITIES}

\section{3-01 Organizational Challenges of New Technology: Implementation of Electronic Health Record Systems In Safety Net Clinics}

Oliver Droppers, MPH (Mark O. Hatfield School of Government, Portland State University); Sherril B. Gelmon, DrPH (Mark O. Hatfield School of Government, Portland State University); Siobhan C. Maty, PhD, MPH (School of Community Health, Portland State University, Mark O. Hatfield School of Government Portland State University)

An emerging issue is the increasing adoption and utilization of health management information systems to improve the quality of health care. Systems such as electronic health records (EHR) can enhance coordination and integration of services, increase organizational efficiencies, improve client and staff satisfaction, and improve population health management. Information technology, along with increasing levels of health information exchange, has the potential to restructure and reform the safety net system, improving delivery of, and access to, health care services.

A multi-year initiative, funded through Kaiser Permanente Northwest and the Northwest Health Foundation, is supporting the implementation of electronic health record systems in three community health centers (CHC) in Portland, OR. An evaluation team is studying the organizational impact and challenges with adoption of an EHR within these CHCs. To date, findings of this study have identified and improved our understanding of the unique and complex organizational barriers and challenges to EHR adoption in CHCs. These key barriers and challenges have been assessed during multiple phases of EHR adoption: (1) Decision-making; (2) Preimplementation; (3) Implementation; and (4) Maintenance and optimization. 
Presentation will highlight findings of the study and explore promising practices for successful EHR implementation and sustained operation in CHCs.

03-02 Open Medicine: The Development of an Open-Acess General Medical Journal

A. Palepu; C. Kendall; S. Choi; S. Murray; D. Giustini; J. Brophy; J. Maskalyk; A. M. Todkill; J. Willinksy (Centre for Health Evaluation and Outcome Sciences, UBC) Open Medicine was conceived in response to the controversy that erupted at the Canadian Medical Association Journal in late 2005 and early 2006. The firing of CMAJ's editor in chief and senior deputy editor in the midst of a dispute over the journal's editorial independence precipitated the resignation of most of the remaining senior editors and editorial board and prompted protest from physicians across the country. The departing editors have used the open-access model and open source journal management and publishing software to launch Open Medicine. This model serves to protect academic freedom and reduces potential interference from vested political and commercial influences. Given the rapid urbanization that is occurring globally in both high- and low-income countries, it is important to have an accessible publishing venue that all health professionals and citizens can access for high quality health information. The creative commons attribution license allows for the reuse and broader dissemination of health information with the condition of attribution. This serves to further the worldwide circulation of health research and analysis. Another feature is the Open Medicine blog where derivative works are created by remixing and mashing up the content with other relevant information and analysis. Open Medicine is part of a broader social movement to open knowledge to a larger world for the benefit of the commons.

\section{3-03 The Detection of At-Risk Populations During Extreme Urban Heat Events: Satellite Remote Sensing and GIS as a Surveillance Tool}

Daniel P. Johnson; George C. Luber (Wright State University)

Geospatial applications have been used for examining a range of disease ecologies involving environmental and socio-economic determinants. This study elucidates the relationship between the satellite detected urban heat island, socio-economic variables and deaths during the 1993 Philadelphia heat wave. The use of satellite remote sensing and geographical information systems is a new approach to this increasing problem. The study demonstrates that the deaths attributed to hyperthermia consistently reside in what is theoretically considered the urban heat island of Philadelphia at the time. Deaths with attributing causes are also examined in this spatial context. Analysis of the thermal and socio-economic variables will be shown using linear statistical techniques as well as artificial neural networks. The relationships between all significant variables will then be discussed in the context of spatial epidemiology and urban health. The primary focus of this research is to effectively develop new and innovative techniques for heat death prevention and surveillance in a changing local and global climate.

\section{3-04 The Effect of Neighborhood Crime on Participation in an Asthma Case Management Program using a GIS \\ Sara Gale; Sheryl Magzamen; John Radke; Ira Tager; Adam Davis (University of California, Berkeley, School of Public Health) \\ Previous studies have demonstrated a direct association between violence and pediatric asthma morbidity. However, exposure to violence remains difficult to quantify. This cross-sectional study examines the association between exposure to}


crime and participation in a community-based asthma case management program in Oakland, CA. Subjects were identified through a school-based asthma case identification survey. Of 265 students eligible for the case management program, $28 \%(\mathrm{n}=73)$ participated. A geographic information system (GIS) model was applied to calculate neighborhood crime exposure using 2003 crime counts reported to the Oakland Police Department. A neighborhood was estimated by calculation of a 1/4 mile circular buffer around each child's residence. The model calculates crime exposure by the addition of proportional block group crime rates within a child's buffer. Logistic regression was used to predict the odds of participation in this population given exposure to neighborhood crime. Preliminary results show that neighborhood crime, as estimated by this GIS model, is not associated with case management participation.

\section{3-05 A Participatory Geomatics Approach to Defining Health-Relevant Neighbourhoods in Ottawa, Canada}

M.-P. Parenteau; M. Sawada; Sam Herold; Elizabeth A. Kristjansson; R. Labonte; M. Calboun; S. Leclair; V. Runnels; A. Musiol; R. Mawby; N. Muhajarine; D. Salisbury; Amira Ali; Marguarite Keeley; Ian Cross; Cliff Gazee (University of Ottawa)

Where we live can impact our physical, mental, and social health. We are investigating the relationship between spatial inequalities and health by developing tools to map the relationship between inequalities of neighbourhoods and health disparities. Geomatics is one of the main components of this project. It provides us with a means to define neighbourhood units and analyze spatial data at this level. Data representing housing, socio-economic and demographic variables from the 2001 Canadian Census at the geographic level of the dissemination area were used as input to spatially constrained cluster analysis and wombling. The results were then integrated with spatial datasets describing physio-social spatial barriers. Clusters were subsequently compared to the neighbourhoods as defined by the Ottawa Real Estate Board's Multiple Listing Services neighbourhood maps and then verified through fieldwork methods. Neighbourhood studies have generally been conducted using one of three epistemological approaches within the social sciences: sociological, functional, or symbolic. Our pragmatic methodology combined the functional with the physical approach, making use of natural forms to define neighbourhoods in conjunction with participatory feedback on symbolic aspects of place provided by from the larger research team comprised of municipal, provincial and local interest groups. We also developed a unique solution to assigning rural regions to satellite communities based on expert knowledge of consumer behaviour and network analysis. The methodology and workflow will be presented in detail.

\section{3-06 A Geobehavioral Analysis of Distances between Illicit Drug and Syringe}

C. T. Williams (University of Illinois at Chicago School of Public Health); D. S. Metzger (University of Pennsylvania Center for Studies of Addiction) (University of Illinois at Chicago)

Geography has become increasingly important in understanding HIV transmission risk and prevention programming strategies. We conduct "geobehavioral" analyses of how drug users' residences, ,drug purchase locations, and injection drug use programs are associated with injection behaviors. Data are from the HIV Prevention Trial Network 037 site in Philadelphia, PA, USA, a randomized study evaluating the efficacy of a network-oriented peer HIV prevention intervention for injection drug 
users. As part of the study, participants were asked the nearest intersection to their residence, where they buy and use drugs, and were given and HIV behavioral risk assessment.

Among the results, participants were more likely to report always cleaning needles before injection when at home or friend/family's home as compared to shooting gallery, abandoned home, car, or public place (chi2 $=10 . .4, \mathrm{p}<0.05$ ); longer distances between home and where drugs are used was related to using injection equipment after someone else had used them $(\mathrm{chi} 2=5.51,[<0.05)$; participants were more likely to get injection equipment from sources other than syringe exchange programs with longer distances between programs and where drugs are used $(\mathrm{chi} 2=$ 14.4, $\mathrm{p}<0.05)$.

Locations where drug injection occurs and their distances from prevention resources may affect safe injection practices.

\section{3-07 A Mobile-Based Housing Locator System}

Mike Bailey (Johns Hopkins University/Center for Communication Programs)

In the 2006 Maryland AIDS Administration's needs assessment housing was ranked [as] the greatest need, above all other service categories. Access to stable housing is fundamental to life itself, the lack of which has obvious implications for urban health and the successful treatment and prevention of many infectious and chronic diseases including HIV/STDs, TB and Diabetes. Calls for an open registry of transitional housing continue and some listings do exist. This has had little impact, for example, on the estimated 5000 PLWH who need to get into treatment. Part of the problem is the need to reconcile rental housing property codes with the existing transitional housing stock. Estimates of $200+$ housing providers operate outside the system in Baltimore City. Another problem is the lack of a housing locater system integrated with treatment and outreach services. Although technology does not create policy, it can often serve as an impetus for change when policymakers can see the possibilities after addressing certain structural constraints. A mobile-based housing locator system accessible over a cell phone could address several objectives related to the accessibility of transitional housing including:

Provide representatives an accessible means for determining what type of housing is available and reserving space for their clients.

Offer a gateway for determining client location, maintaining treatment regimens, and keeping appointments.

Provide an impetus for reconciling existing rental housing codes with existing need. Provide an incentive for operators of transitional housing to join an open registry and address some of the safety requirements for providing housing.

The technology used in the development of the housing locater system (Java for Mobile Devices) is accessible by the majority of cell phone models. While the web interface for the Housing Administrators is based upon open source technology (PHP/MySQL).

\section{3-08 Automatically Updated Population Health Report for the Toronto Homeless Population}

B. Ramin; T. Svoboda; I. Carrion (Client Access to Inegrated Services and Information (CAISI))

The Client Access to Integrated Services and Information (CAISI) is an open source case management and electronic medical records (EMR) system aimed at integrating service 
provision to those who are homeless (www.caisi.ca). Using CAISI records we have developed a publicly available online population health report providing real time information on those who are homeless in Toronto and enrolled in shelter bed programs utilizing CAISI. The population health report contains 14 variables under the headings of demographics, mortality, prevalence and incidence of major medical conditions, and prevalence of major mental illness. Diagnoses are entered by clinicians at the point of care into CAISI EMR software using ICD-10 coding. These codes are then aggregated and anonymous data is presented on the CAISI website. The report was built with feedback from clinicians, community and municipal agencies and members of CAISI's client advisory board. The report is continuously being evaluated and we plan to expand the variables reported and the number of agencies contributing to the report. Our project intends to enhance the community's ability to gather data on the homeless population that can be used by decision makers, academics, and advocates to effect positive social change leading to the end of chronic homelessness.

\section{3-09 Interactive Health Technology Models: Innovative Education in Urban Communities}

Sarah Wessler; Jeb Weisman; Anne Steinfeld (Harlem Children's Health ProjectLehman Brothers Health Promotion Learning Lab)

The Lehman Brothers Health Promotion Learning Lab (HPLL) is a unique health education resource in the heart of Harlem, NY. The HPLL components leverage an array of collaborative information and audio-visual systems to teach students and their families about personal, public and community health. Located in the Harlem Children's Zone (HCZ) Promise Academy Charter School, the HPLL is a model facility that aims to empower young people in the HCZ community to take control of their personal health and to become advocates for a safer and healthier neighborhood, community, and planet. The HPLL incorporates a model curriculum, pedagogy, and student internship program. Student interns from NYC assist with modes of education, teach lessons, and act as peer mentors for visiting students.

Field-tested interactive hands-on health education materials are integrated into the HPLL to provide students a workshop model in which to learn and experiment. Based on the needs of the Harlem community, the HPLL has designed several custom computer interfaces and lesson plans to address topics including: nutrition, diabetes, anatomy, hygiene, asthma, and sexual health. The HPLL is an ideal model for replication in a variety of settings including school- based health centers, community-based health centers, and children's hospitals.

\section{3-10 An Alternative Model to Bridge Urban Health Disparities and RHIO Benefits} John Crilly, PhD, MPH, MSW

Ann Marie White, Ed.D.

Robert Keefe, PhD, MSW (University of Rochester Medical Center and State University of New York at Buffalo School of Social Work)

Rochester, NY has both a high density of poor residents and a renowned healthcare system. Poverty and environmental risk factors can heighten health disparities by increasing the need for health services while simultaneously decreasing access. Consequently, disenfranchised residents seek care in expensive emergency settings or not at all. Isolation from the healthcare system can then limit the potential of benefits from regional health information organizations (RHIOs).

However, this group is more likely to be involved with other community agencies or systems, such as criminal justice, courts, social services, and specialized education. 
These entities have extensive data systems which could be helpful in advancing community-based health planning. Linking together disparate data systems in a RHIOlike manner could provide a mechanism to help identify and decrease health disparities.

We have developed a theoretical Alternative Epidemiology System (AES) model to link community-based non-health agencies. We will present and describe the AES model and focus on the importance of the first critical steps: extensive community trust-building and preparation around common goals. Paired community involvement is the critical component which can help design tailored interventions to meet acceptability, cost, training, and supervision requirements and have direct relevance to individuals' health care and resulting health status.

\section{3-11 Promoting Smoking Cessation among Methadone Users}

Oleg Lapshin, MD, MPH, Postdoctoral fellow, Joseph Finkelstein, MD, PhD, Assistant Professor (University of Maryland)

Study Aim: Up to $90 \%$ of patients visiting methadone clinics smoke. There are different approaches that can be used for promoting smoking cessation in these clinics. We conducted a pilot study on the feasibility of using tablet computers for anti-smoking education in a methadone center.

Method: We asked 35 methadone-treated patients from Baltimore low income inner city area who smoke to use tablet PCs for $45 \mathrm{~min}$ - $1 \mathrm{hr}$ in order to study a computer program on smoking hazards. The computer program was guided by adult learning theories and provided interactive self-paced education tailored to user comprehension rate. We collected data on their smoking status, and administered a smoking hazards knowledge questionnaire before and after the intervention. The questionnaire consisted of 35 statements about smoking. Patients responded whether a statement was true or false.

Results: A sample consisted of 27 women $(77.1 \%), 8$ men; 45.4 to 67 years old. Most of them were unemployed (27 subjects, $77.1 \%$ ). They smoked for $23.2 \mathrm{t} / \mathrm{-}$ 10.2 years and 25 subjects $(71.4 \%)$ smoked 1 pack a day or more. According to Prochaska's Stage of Change Scale, 12 patients $(34.3 \%)$ were in a preparation or contemplation stage, and $23(65.7 \%)$ were in a pre-contemplation stage. Twenty patients $(57.1 \%)$ discussed their smoking at least once with their doctors, although this was not connected with their knowledge about smoking hazards or their stage of change. Knowledge on smoking hazards significantly increased from 14.2+/- 2.9 points to $20.1+/-3.0$ points, $\mathrm{p}<0.0001$. For 27 subjects $(77.1 \%)$ it was not complicated at all to use the computer, most of them (32 persons, $91.4 \%$ ) rated the program as good or excellent.

Conclusion: Tablet PC was a feasible and effective mean of education about smoking hazards and was favorably accepted by patients. Significance: Antismoking education that could be successfully implemented in methadone clinics has high potential to be used in methadone clinics for health education.

\section{3-12 Using Large Disparate Data to Predict Trends in Intimate Partner Homicide}

Darryl W. Roberts, PhD(c), MS, RN (University of Maryland School of Nursing) Like most other types of homicide, a large proportion of intimate partner homicides (IPH) occur in cities. However, because IPH accounts for only $7 \%$ of all US homicides, many consider it a relatively small urban health problem. Consider, however, that in 2004 the War in Iraq claimed the lives of 848 Americans, while 1,544 Americans died at the hands of their intimate partners. A significant proportion of those crimes occurred in US cities. Because of the lack of priority given to this crime, social scientists know little about its predictors. What we do know comes largely from a 
combination of small cross-sectional or larger, geographically limited, time-series studies.

In the current study, the researcher combined 62 publicly available datasets encompassing 20 years of rich national data from the FBI, BLS, US Census, and the Association of Religion Data Archive in an effort to identify sociodemographic, geographic, and temporal predictors of IPH.

Among the surprising findings: the IPH rate is decreasing, but the proportion of female victims is increasing; the geographical and temporal distributions of IPH are more like that of suicide than that of homicide; and religious congregation density strongly affects the incidence of IPH.

\section{NEIGHBORHOOD LEVEL INFLUENCES ON HEALTH}

\section{4-01 Individual Stressors, Neighborhood Risk Factors and Caregiver Depression} Joan Kub, PhD, APRN, BC; Jacky M. Jennings, PhD, MPH; Jennifer Walker, MHS; Arlene Butz, ScD, RN, CPNP (Johns Hopkins University)

INTRODUCTION: There is a growing interest in neighborhood effects on health. The goal of this study was to assess the contribution of macro-level social factors and individual risk factors to depression in a sample of 231 urban caregivers of children with persistent asthma.

METHODS: Caregivers were interviewed regarding depressive symptoms using the Center for Epidemiological Studies-Depression Score (CESD), demographic factors (education, income, employment), individual experiences of community violence (self-reports of feeling unsafe, seeing violence personally or reporting that their child saw violence), and perceptions of child's asthma control. Out of control was defined as symptoms at some/a lot or always. Census tract-level data about crime rates, poverty, unemployment, vacant houses, and home ownership will then be incorporated into the analysis.

RESULTS: Children were primarily African American (92.6\%), male (60.6\%) and Medicaid insured (83\%). Most parents reported their child's asthma as being in control $(73.59 \%) .25 .65 \%$ of the caregivers had scores of 16 or greater on the CESD, indicating depression. Individual experiences of community violence were positively associated with depressive symptoms. Lower education (less than high school degree) and unemployment were also associated with depressive symptoms while asthma control status was not related to depression. Macro-level variables will be added to the model.

CONCLUSIONS: Multilevel modeling is an important approach that may contribute a greater understanding of health outcomes.

\section{4-02 Urban Aging: Bridging the Divide Between Health Care and Aging Services to Address Neighborhood-based Disparities among Older Urban Adults}

Marianne C. Fahs, PhD, MPH; Nina S. Parikh, PhD, MPH; Dana Friedman, MPH; Mathew Caron, MS (Brookdale Center on Healthy Aging and Longevity)

Background. Innovative models for targeting effective prevention services among urban community-based older adults are critically needed. Yet a paucity of studies differentiates health status and use of evidence-based preventive services by race/ ethnicity and neighborhood. This study compares utilization predictors by race/ ethnicity and neighborhood among seniors attending senior centers, a potentially vital structural component of urban aging policy. 
Methods. The research design is cross-sectional. In-person interviews were conducted using standardized health status instruments (e.g. SF36; PHQ-2). A stratified sample of seniors was selected to represent ten racially/ethnically diverse neighborhoods in NYC. The sample $(n=483)$ is composed of White non-Hispanics $(\mathrm{n}=78)$, Latino/Hispanics $(\mathrm{n}=95)$, African Americans $(\mathrm{n}=126)$, Asian/Pacific Islanders $(n=173)$, and Others $(n=11)$. Logistic regression models were used to estimate the effects of neighborhood and race/ethnicity on preventive service use, controlling for socioeconomic and health characteristics.

Results. Interestingly, senior center attendees are representative of the US population of community-based older adults, based on nonsignificant differences in physical and mental health scores (SF36). Yet important differences by race/ethnicity were identified. For instance, $24.2 \%$ of Latinos were depressed, compared with $15.1 \%, 13.3 \%$, and $9.0 \%(\mathrm{p}<.001)$ among Blacks, Asians, and Whites, respectively. However, after controlling for health status, place of birth, age, education, access, and insurance status, logistic results reveal neighborhoods to be highly significant predictors of specific preventive service use, e.g. colonoscopy and mammograms, mediating the effects of race/ethnicity.

Discussion. New policy incentives for neighborhood-based organizations such as senior centers are recommended in order to address the complex interactions of race/ethnicity with neighborhood-based determinants of healthy urban aging.

\section{4-03 The Role of Poverty in Explaining Geographic Variation in Breast Cancer Survival Among Older Women.}

Mario Schootman; Min Lian; Donna B. Jeffe (Washington University School of Medicine)

This study's purpose is to examine small-area geographic variation in breast cancer survival among women age 66 or older and the role of census-tract-level poverty rate and other demographic, clinical, and healthcare-related variables. Data are from NCI's 1992-1999 Surveillance, Epidemiology, and End Results (SEER) program (Atlanta, Detroit, San Francisco-Oakland, Seattle-Puget Sound, and the state of Connecticut), 1991-1999 Medicare claims files, and 1990 census. We used an elliptical spatial scan statistic to identify areas of increased or reduced breast cancer survival. Of 27,936 women, 2817 died of breast cancer. Breast cancer survival varied across these SEER programs $(\mathrm{p}<0.001)$. In the Detroit area, one cluster of worse-than-expected breast cancer survival was identified in unadjusted analysis $(R R=1.60)$. In the Atlanta area, one cluster of worse-than-expected $(\mathrm{RR}=1.81)$ and one cluster of better-than-expected $(\mathrm{RR}=0.72)$ breast cancer survival were identified. In adjusted analysis, all clusters were explained by poverty rate $(\mathrm{p}>0.05)$. Little explanatory power was accounted for by the other variables after controlling for poverty rate. There were no clusters of increased or reduced breast cancer survival identified in the other three SEER areas. In conclusion, local poverty rates played a key role in explaining the geographic variation in breast cancer survival in Detroit and Atlanta.

04-04 Health Disparities and the Public Agenda: Building Capacity Among Community Activists, Building Relationships with Local Media

Josephine Crisostomo; Blanca Figueroa; Ediss Gandelman; Vilma Lora; Chrasandra Reeves; Clara Savage; Sherrie Flynt-Wallington; K. Viswanath (Dana-Farber Cancer Institute)

Despite the documentation of profound health disparities among different socioeconomic, racial and ethnic groups, there has been surprisingly little attention to 
the issue in the public arena. Public mobilization is critical for changing social and health policies to eliminate health disparities. Though it is agreed that news media play a critical role in setting the public and policy agenda, our recent survey of health reporters showed that health disparities is not a priority topic for them. This presentation reports on a community-academic collaboration to train community activists and leaders to promote the public agenda on health disparities through strategic communications workshops. The workshops were conducted under the aegis of NCI-funded Community Networks Project, MassCONECT (Massachusetts Community Networks to Eliminate Cancer Disparities through Education, Research and Training) in Boston, Lawrence and Worcester. The workshops featured panel discussions with local reporters and community activists, sessions on how to develop health campaigns and work with local media in promoting their causes through lectures, discussions and hands-on exercises. Each workshop was evaluated for quality and effectiveness. The workshops serve as exemplars of strong partnership between the academic researchers at Harvard and Community Partners to promote the public agenda on health disparities.

\section{4-05 Participatory Research and Evaluation: Collective Community Action for Urban Health Programming}

Ram Saran Adhikari (National Resource Center, Nepal)

Introduction: This program aims at building social infrastructure and linking slum communities by participating them in health programs. Participants will understand how potential of slum communities can be utilized for effective implementation of health programs.

Methods: Slum based networks and health care providers helped in identifying unaccounted slum areas and in understanding health seeking behavior of the slum population. Population living in the slum areas was encouraged to participate in awareness programs and sensitization work in the slums.

Results: 25 health care providers worked in 10 different slum areas around Kathmandu Valley. The work team members were successful in empowering slum families to adopt healthy behaviors and avail health services. The data indicates increase in TT immunization from $29.23 \%$ to $75.98 \%$, timely initiation of breast feeding from $27 \%$ to $79 \%$; between November 2005 to January 2007. The health team members negotiated with government officials and elected representatives to access health services and basic services like water. Members also organized drama based informative programs to encourage more people to participate.

Conclusion: This program is focused on strengthening social infrastructure in slums by stabilizing community level institutions and linking them with city level programs. This program is considered as one of the most effective urban health programs.

\section{4-06 Individual and Neighborhood Level Predictors of Depression in a Longitudinal Study of older Residents of New York City}

John R. Beard; Shannon Blaney; Sandro Galea; David Vlahov; Danielle Ompad (Center for Urban Epidemiologic Studies)

This prospective cohort study followed residents of New York City (NYC) 50 years of age or older over two waves of telephone interviews. 1411 participants were interviewed in 2005 and 807 re-interviewed in 2007. 99 participants died between interviews (61.5\% follow-up rate). Validated instruments were used to measure key variables and this information was linked to a comprehensive geo-spatial database of environmental characteristics of the 59 NYC community districts. GEE were used to identify individual and neighborhood level factors predicting both new onsets of 
depression in participants who were diagnosis free at baseline, and change in depression score between the two interviews.

Depression onsets were increased for participants with lower education, lower income, higher scores on personality trait neuroticism, higher levels of recent life stressors, increased physical limitation and Hispanic background. Significant neighborhood level predictors included percentage of buildings with missing/broken windows, number of assaults reported and lower collective efficacy score. In multivariate analysis, new onsets were significantly associated with neuroticism, lower baseline income and buildings with missing/broken windows. When change in depression score was used as the outcome, similar associations were found. However, gender, social networks and support and several more neighborhood level variables were also significant predictors of worsening score in multivariate analysis.

This study highlights the important influence of the physical and social environment on the mental health of the elderly.

\section{4-07 Pathways Connecting Neighborhood Influences and Mental Well-Being: Socioeconomic Position and Gender Differences}

J. Burke; P. O’Campo; C. Salmon; R. Walker (University of Pittsburgh)

A gap remains in knowledge about the specific pathways between the environment and mental well-being. Many existing studies lack appropriate data to explore the mechanisms by which residential neighborhoods affect mental health and fewer yet have attempted to gain the perspective of the residents about pathways from the environment to mental well-being. The primary goal of this research was to examine the range of neighborhood influences felt to be related to mental well-being. Specific attention was given to whether and how participant gender and socioeconomic position (SEP) differentially affect perceptions. Concept mapping, an intensive structured conceptualization process that produces a framework for how a group views a particular topic, was used. A strength of the method is that participants contribute to the generation and analyses of data, thus, ensuring that the final products represent their perspectives versus that of the researcher. 37 low and nonlow income men and women from Toronto, Canada participated in the concept mapping sessions. They identified 120 neighborhood characteristics perceived to be related to mental well-being and selected a final six cluster concept map. Notable difference in cluster importance by participant SEP status exists. While no overall differences were observed for males verses females, further stratification by both participant SEP and gender indicate that perceptions are not uniform. Participants were able to articulate and diagram how the cluster domains are related to each other and to good mental well-being. These diagrams offer insight into the pathways connecting neighborhoods and mental well-being and the SES and gender stata differences identified. These findings are important for documenting the mechanisms by which neighborhoods affect mental health and inform testable hypotheses for future qualitative and quantitative, multi-level, research on this topic.

\section{4-08 Urban Neighbourhoods and Health: Exploring Regional and Gender Differences in Neighbourhood Deprivation and Self-Reported Health}

Heather L. White, M.Sc; Flora I. Matheson, PhD; Rahim Moineddin, PhD; James R. Dunn, PhD; Richard H. Glazier, MD, MPH (Centre for Research on Inner City Health)

There is increasing recognition that place can be an important determinant for health, and subsequently for disease. While there is considerable evidence to suggest 
that neighbourhood deprivation exerts an independent effect on health outcomes, there is little evidence as to whether these effects vary between men and women and across geographic regions. Therefore the purpose of this investigation was to explore regional and gender differences in neighbourhood deprivation and self-reported health. Data from the Canadian Community Health Survey, a nationally representative survey consisting of individuals living within 25 urban CMA's ( $N=120,492)$ were combined with area level data from the 2001 Canada Census. The analysis was stratified by region and gender and the relationship between neighbourhood deprivation and the odds of reporting fair/poor health were analyzed using multilevel hierarchical regression. Individuals living in neighbourhoods with higher material deprivation were significantly more likely to report poor health, independent of individual level demographic and socioeconomic characteristics. Significant gender differences exist among the associations between material deprivation and self-reported health, however, the magnitude and direction of gender differences vary between regions. These findings are novel to the field, indicating that the influence of neighbourhood deprivation on health is both gender and place specific.

\section{4-09 Neighbourhood Characteristics and Mental Wellbeing: Lived Experiences of Residents in Toronto, Canada}

A. Ritchie; A. Jabbar; N. Haque; J. Parsons; A. Scott; G. Singh; J. Ramsay; Q. Zaidi; P. Balasubramaniam; R. Nisenbaum; J. Dunn; P. O’Campo (University of Toronto) Introduction: There is increasing evidence that the places where people live can influence their mental wellbeing however few studies have actively involved local residents to understand their perceptions of the potential pathways from neighbourhoods to mental wellbeing.

Methods: Concept mapping was conducted with residents from 5 Toronto neighbourhoods representing low income and non-low income socio-economic groups. These residents participated in two sessions per neighbourhood in small groups. The first session of brainstorming was to generate neighbourhood characteristics that the residents felt influenced their mental wellbeing. A couple of weeks later, residents returned to individually sort these neighbourhood items and then rate them as to their importance with respect to good and poor mental wellbeing. The data from the sorting process were analyzed using concept mapping methodology.

Preliminary Results: Data from 5 Toronto neighbourhoods were used to generate conceptual maps of neighbourhood characteristics that influence their mental wellbeing. While several similarities existed between the perceptions of residents among the different neighbourhoods, some important differences were revealed.

Conclusions: These findings will contribute to the limited literature on the relationship between neighbourhoods and mental wellbeing, as well as present valuable findings towards informed urban planning in a Canadian context.

\section{4-10 Environmental Risk Factors For Asthma Emergency Care: A Multilevel Approach For Ecological Study}

MaryAnn Sorensen Allacci, PhD (Project for Environmental Health Knowledge and Action (PEHKA))

This study describes a model for evaluating environmental factors to explain residential patterns of adults using New York City's Harlem Hospital Asthma Emergency Department (Asthma ED) services. A multilevel design incorporated methods for hypothesis testing using geographic information systems (GIS) to map 
data from Harlem Hospital Center and other sources; to define geographic levels of analysis; to measure variables on building and street segment levels; and to analyze variables for their affects on the outcome (asthma visits) while accounting for the influence of other variables.

Spatial data analysis was also performed with GIS and revealed significant clusterings of addresses of Asthma ED visitors. Models were then specified to explain how building, segment, and combined building and segment level variables affected geographic variations in frequency of Asthma ED visits. Results showed that asthma-related housing violations, segment density, and residence in corner buildings were among variables found to be significant predictors of asthma visits. The study supports the benefits of examining environmental factors at different spatial levels for developing a deeper understanding of ecological conditions linked to Emergency Department use.

\section{4-11 Urban Environment and Perceived Hhealth: Contextual Analysis of a Pilot Study in Paris (France)}

Emmanuelle Cadot; Alfred Spira (INSERM U 822)

Introduction: Paris is characterized by its heterogeneous demographic composition, the disparate texture of a dense urban environment and very high population densities. There are cities in the city. In this context, we hypothesize that residence places determine partly population health and its behaviors, especially at the neighborhood level.

Methods: The study was based on a phone survey among a sample of the Parisian population. We designed a complex sample protocol with three degrees random sampling. The questionnaire included modules about self-reported measures of height and weight, poverty, smoking status, perceived health and social capital. The data analysis is based on multilevel models to take account of the hierarchical structure of the data. Between November 2006 and December 2006, computerassisted telephone interviews were completed by 732 landline telephone subscribers and 118 exclusive cell phone subscribers.

Results: The stratification of the city is based on a socio-economic typology of the eighty districts of Paris. First results show that perceived mental health is better in the wealthiest neighborhoods. Results concerning the contextual analysis will be presented.

Discussion/Conclusion: This pilot study allows gathering statistical data concerning perceived health and behaviors among the Parisian population. The first analyses help to better understand the individual determinants of behaviors and allow taking into account neighborhoods characteristics. The comparisons between districts constitute the originality of this study because this kind of data did not exist until now.

\section{4-12 The Multi-Disciplinary Outreach Team: A Unique Approach to Bringing Healthcare to Toronto's Street Homeless}

Sarah Innis (Multi-Disciplinary Outreach Team (M-DOT))

Toronto's Street Needs Assessment of 2006 indicated that a large proportion of homeless people experience severe mental and physical health issues as a barrier to finding and maintaining housing.

The Multi-Disciplinary Outreach Team (M-DOT) was developed late last fall to address this barrier. The program is a unique partnership funded by the City of Toronto which brings together 5 homeless service agencies. The team is comprised of an outreach worker, a registered nurse, a housing worker, two part-time psychiatrists and a concurrent disorders specialist. We work with the most marginalized of homeless 
clients: those living outside, suffering with mental, physical health and substance use issues. The service is client driven and not considered a crisis service.

Our referrals come from other outreach service providers who refer at the point when health or substance use issues prevent forward movement with a client. The goal is short term intensive case management with eventual linkage to existing supports and long term case management programs.

We recently completed our first client needs assessment and preliminary evaluation of the program. The results provide a snapshot of our clients and highlight the interventions most critical to address their needs. We have identified ways to streamline services and maximize access for our clients.

The project has achieved some significant successes for clients who have achieved access to income supports, housing, and a range of health and substance use services.

04-13 Creating Factors to Assess Neighborhood Impact on Health of Black Urban Elders Priscilla T. Ryder, MPH, PhD; Ann L. Gruber-Baldini, PhD (University of Maryland School of Pharmacy)

Background: There is increasing evidence of neighborhood effects on elderly urban residents' health. It is unclear which neighborhood attributes are most determinative and a useful and meaningful way to aggregate them is needed.

Methods: Multiple neighborhood indicators were examined in 95 African Americans ages 60 and older living in 16 Baltimore, Maryland neighborhoods. Principal component analysis was performed to determine an appropriate number of factors, and factor analysis using a promax rotation was performed on 22 neighborhood variables describing demographic composition, physical appearance, socio-economics, and cohesion.

Results: A four-factor solution was derived, accounting for $86.3 \%$ of the variance. Factor 1 (Deprivation) measured vacant/abandoned properties, proportions in poverty and unemployed, numbers of neighborhood associations, owner-occupied residences, area covered by tree canopy, and neighborhood income equality. Factor 2 (Economic Stability) contained items on residential foreclosures, reports of rat activity, higher education, and residential rehabilitations. Factor 3 (Neighborhood Instability) contained proportions of residential evictions and families not headed by married couples. Factor 4 (Interpersonal Relations) represented crime rate, racial segregation, and percent ages 2544. With all factors considered simultaneously in a logistic regression model, Factor 1 was significantly associated with self-rated health of older Blacks $(\mathrm{OR}=0.983,95 \% \mathrm{CI}$ 0.969, 0.998), while Factor 2 was nearly significant (OR $=0.9732$, 95\%CI 0.916, 1.001). Neighborhood effects were attentuated when individual-level factors (depression, age, gender, number of physician visits, and pain) were included in the model.

Conclusion: It is possible to create meaningful aggregate neighborhood quality measures, although in this analysis, influence of these factors on self-rated health may be mediated by individual-level variables.

\section{4-14 Recent Incarceration and Use of a Supervised Injection Facility in Vancouver, Canada}

M.-J. S. Milloy; E. Wood; W. Small; R. Zhang; J. Montaner; T. Kerr (Centre for Excellence in HIV/AIDS)

Background: There are concerns that Vancouver's supervised injection facility (SIF) for injection drug users (IDU) is an amplifier of drug-related crime. Therefore, we sought to evaluate the possible association between SIF use and recent incarceration among IDU. 
Methods: We examined incarceration experiences among participants in the Scientific Evaluation of Supervised Injecting (SEOSI) cohort. Correlates of recent incarceration were identified using generalized estimating equations (GEE).

Results: There were 1,090 individuals included in this analysis, of which 773 $(70.91 \%)$ were male. At baseline, 157 (14.46\%) reported incarceration in the previous six months; $879(80.64 \%)$ reported a history of incarceration. In the univariate GEE analysis, recent incarceration was not associated with frequent SIF use (Odds ratio: $0.84, \mathrm{p}=0.06)$. In the multivariate GEE analysis, factors strongly associated with recent incarceration included: unstable housing (AOR: 1.77, $\mathrm{p}<0.001$ ); drug dealing (AOR 1.71, $\mathrm{p}<0.001$ ); and non-fatal overdose (AOR 1.54, $\mathrm{p}<0.01$ ).

Conclusions: Although incarceration is a common experience among local IDU, our analysis does not provide evidence to support claims that use of the SIF is increasing involvement in drug-related crime in the community. Our findings also indicate that incarceration continues to be associated with high-risk behaviours.

\section{4-15 Neighborhood Effects on Children's Health: The Role of Subjective and Objective Evaluations of the Neighborhood Context}

Nicole J. Schaefer-McDaniel (Graduate Center, City University of New York)

Neighborhood research, the body of work exploring the link between the neighborhood context and residents' behaviors, has received growing attention in recent years. Although this literature is rapidly expanding, there is currently no consensus on how researchers should measure the neighborhood space. While numerous measures have been introduced, none of these recognize children as possible informants of neighborhood conditions. Also, little remains known about the relationship among these assessments or how well each of these measures predicts outcomes such as residents' health.

Building on qualitative research, 126 preadolescent children in three New York City neighborhoods and their parents completed surveys on their perceptions of neighborhood safety, quality, social disorder and physical disorder. The surveys also assessed the children's mental and physical health. Neighborhood observations were then conducted by outside raters and census data at the block group level of aggregation were collected.

Results suggest that children and parents showed some overlap in their perceptions of neighborhood conditions but these subjective ratings did not relate to the neighborhood observations and census data. Subjective ratings of neighborhood safety mediated the relationship between neighborhood disorder and neighborhood quality and child-rated perceptions of neighborhood conditions predicted their ratings of mental health. Neither parents' neighborhood perceptions, neighborhood observations, nor the census data related to children's mental health. On the other hand, observed physical disorder and child-rated neighborhood safety predicted children's physical health.

This study provided insight on the relationship among various neighborhood measurements and also found that children's perceptions of neighborhood conditions related best to their health. Future research should refine neighborhood assessment and involve children more actively in research concerning them.

\section{4-16 Neighborhood Effects on Birth Weight: An Exploration of Psychosocial and Behavioral Pathways}

Ashley Schempf; Donna Strobino; Patricia O’Campo (Johns Hopkins Bloomberg School of Public Health)

Neighborhood characteristics have been proposed to influence birth outcomes through psychosocial and behavioral pathways, yet empirical examination is 
lacking. Using data from an urban, low-income sample, this study examined the impact of the neighborhood environment on birth weight and evaluated mediation by psychosocial and behavioral factors. The sample included 726 women who delivered a live birth at Johns Hopkins Hospital between 1995 and 1996. Census tract data were used to create a principal component index of neighborhood risk based on racial and economic stratification (\% Black, \% poverty), social disorder (violent crime rate), and physical deterioration (\% boarded-up housing) (\&\#945;= 0.82). Information on sociodemographic, psychosocial, and behavioral factors was gathered from a postpartum interview and medical records. Random intercept multilevel models were used to estimate neighborhood effects and assess potential mediation. Controlling for sociodemographic characteristics, a standard deviation increase in neighborhood risk conferred a 76 gram birth weight decrement $(\mathrm{p}=$ 0.01 ), with a $\sim 300$ gram difference between the best and worst neighborhoods $(76 \mathrm{~g} * 4 \mathrm{SD})$. Although stress (daily hassles), locus of control, and social support were related to birth weight, their adjustment reduced the neighborhood coefficient by only $12 \%$. In contrast, the neighborhood effect was reduced by an additional $30 \%$ and was no longer significant after adjustment for the behavioral factors of smoking, drug use, and delayed prenatal care. These findings suggest that neighborhood factors may influence birth weight by shaping maternal behavioral risks. Future studies should examine more direct measures of neighborhood stress, such as perceived neighborhood disorder, and evaluate alternative mechanisms by which neighborhood factors influence behavior (e.g., social norms and access to goods and services).

\section{4-17 Neighborhood Crime Incidents, Perceived Neighborhood Crime and Safety, and Adherence to a Walking Intervention Among Midlife African-American Women}

April Oh, MPH (University of Illinois at Chicago School of Public Health); Shannon N. Zenk, PhD (University of Illinois at Chicago College of Nursing); JoEllen Wilbur, $\mathrm{PhD}$ (University of Illinois at Chicago College of Nursing); Richard Block, PhD (Loyola (University of Illinois, Chicago)

Despite great interest in effects of neighborhood crime on physical activity, few studies have included both objective and perceived measures of neighborhood crime or examined effects of crimes beyond violent crime. This exploratory analysis examines relationships among police-reported neighborhood incidents of crime, perceptions of neighborhood crime/safety, and adherence to a physical activity intervention among midlife African-American women. The sample was comprised of 252 women from metropolitan Chicago who participated in a home-based, moderate-intensity walking program. Counts of police-recorded crime incidents within a one mile radius buffer of the participant' home were measured using GIS. Crimes included homicide, rape, assault, robbery, drug activity, prostitution, vandalism, and weapons possession; outdoor and total incidents were examined separately. Citizen-reports of gun shots fired were also examined. Perceptions of neighborhood crime/safety were measured prior to intervention delivery. Adherence was measured as the percentage of recommended walks completed, reported in walking logs and recorded on heart rate monitors. Preliminary analyses show statistically significant correlations between perceived and objective measures of crime/safety, but few associations between objective or perceived neighborhood crime/safety and adherence. These analyses provide new insights into the effects neighborhood crime and perceived neighborhood crime/safety on midlife African-American women interested in becoming more physically active. 


\section{4-18 What Does Access to Maternal Care Mean Among the Urban Poor? Factors Associated with Use of Appropriate Maternal Health Services in the Slum Settlements Of Nairobi, Kenya \\ Jean-Christophe Fotso; Alex Ezeh, Nyovani Madise; Abdhalah Ziraba; Reuben Ogollah (The African Population and Health Research Center (APHRC))}

A key feature of urbanization in Africa is that urban poverty is high and is growing rapidly. To improve heath indicators nationwide, a close attention should be paid to the growing urban poor. This study uses unique data from a maternal health project conducted in two slums (Korogocho and Viwandani) of Nairobi, Kenya, to: 1) identify the factors which influence the choice of place of delivery; and 2) formulate recommendations aimed at improving maternal health among the urban poor. The dependent variable is a three-category ordinal variable; and ordered logic models are used to quantify the effects of selected covariates. Although $70 \%$ of women reported health facility delivery, only $48 \%$ delivered in a facility with skilled provider. This suggests that health indicators for the slum populations may be misleading if the quality of service is not taken into consideration. Besides education and wealth, the factors associated with health facility deliver included area of residence, being advised during antenatal care, pregnancy wantedness, and parity. Interventions to improve the health of urban poor women should include improvements in the provision of, and access to, quality health services, and health promotion campaigns, with special focus on poorest, lower-educated and higher parity women.

04-19 Poor-Rich Inequalities in the Health and Survival of Urban Children in Bangladesh Subir Saha, PhD candidate; Michelle Kouletio, MPH (Concern Worldwide Bangladesh) Despite significant improvements in child survival in recent decades, infant and under-five mortality in Bangladesh remain unacceptably high with strong urban-rural differentials. In Bangladesh, nearly one in nine children dies before reaching age five. Neonatal mortality contributes to more than sixty percent of the infant mortality and almost half of all under-five deaths. Like other developing countries, child survival status has been consistently better in urban Bangladesh than that of rural areas. Reducing poor-rich inequalities in the health and survival of children within developing countries is a perquisite for achieving the MDG-4. However, according to a recent study on the urban poor in Bangladesh, using the data from Bangladesh Demographic and Health Survey, 2004 and applying Principal Component Analysis (PCA) for determining the relative economic position of the household and using a synthetic cohort Life Table approach for mortality estimates, it is evident that urban poor have the worse health situation than the nation as a whole. The urban extreme poor have the highest under-five, infant and neonatal mortality rate at 138,111 and 57 per 1000 live births respectively; these are much above the rural and national level estimates. While under-five, infant and neonatal mortality rate for urban richest are at 63, 60 and 37 per 1000 live births respectively. So to achieve the child survival millennium development goal in Bangladesh must need to reduce these enormous differences in health status and survival chances among urban children.

\section{4-20 The IMPACT Studies: Organization and Rationale for Multilevel Intra-Urban Studies for Marginalized Populations}

Danielle C. Ompad; Sandro Galea; Grant Marshall; Crystal M. Fuller; Linda Weiss; John Beard; Christina Chan; David Vlahov (New York Academy of Medicine, Center for Urban Epidemiologic Studies)

Illicit drug use in urban neighborhoods remains a major public health problem. The neighborhood-level determinants of the different stages and consequences of illicit 
drug use are poorly understood. Study designs that allow for intra-urban comparisons of drug use help us understand the role of neighborhood-level factors address these questions. Studies of neighborhood influences are typically concerned with proper selection of neighborhood units that are meaningful to local residents; such studies have often randomly sampled households within the urban areas of interest. Studies among marginalized populations such as illicit drug users are primarily concerned with identifying and recruiting these frequently hard-to-reach groups. Sampling approaches for studies of hidden populations have primarily included chain referral, snow ball and more recently, respondent driven sampling. Limited academic attention has been paid to the fusion of the two concerns, namely, sampling marginalized populations within the context of well characterized neighborhoods as sampling frames of interest. The IMPACT studies in New York City were designed to facilitate consideration of neighborhood characteristics and their potential role in shaping the health of drug using populations. This presentation will discuss the organization and rationale for the IMPACT Studies, with a focus on the challenges faced, and solutions devised, in sampling marginalized populations within focal neighborhoods in an urban area.

\section{4-21 Drug Markets and the Built Environment: The Development of an On-site Observational Tool}

Michael S. Oldham (The George Washington University School of Medicine and Health Sciences) (Johns Hopkins Bloomberg School of Public Health, Department of Epidemiology); Jacky M. Jennings (Johns Hopkins School of Medicine, Department of Pediatrics)

Drug markets may act as a causal pathway through which features of the built environment affect STI/HIV vulnerability for urban youth. To measure drug markets and the built environment, on-site observations may be necessary to capture attributes that are not measured in census or administrative data.

Methods. Based on a systematic, ordered sampling strategy from a frame of 486 census block groups (CBGs) (with greater than the lowest 25th percentile in gonorrhea rates per CBG), 65 CBGs were selected and ordered by gonorrhea rate, percent below poverty and geography. From the 65, 4 CBGs were randomly selected to pilot an on-site observational tool. Within each CBG, $25 \%$ of face-blocks and alleys were selected for observation. Measures included land type and counts of abandoned structures, trash, drug paraphernalia, and condoms.

Findings and Conclusions. Of the 33 block faces assessed, 30 (91\%) were primarily residential. Eight of the block faces (24\%) had abandoned, boarded up, and/or burned out buildings. Almost $25 \%$ of the block faces had minor or major structural damage. Based on analysis of specific trash counts, statistically significant differences in the mean number of alcohol containers were found between the CBGs. This pilot work highlighted several methodological and analytical issues that are important in exploring the built environment's measurement definitions, data collection logistics, and the implications of data aggregation.

\section{4-22 Community Socioeconomic Composition and Cigarette Smoking Initiation, Current Use and Intensity: A Multilevel Analysis}

Gary L. Ellison; Shanita Williams-Brown; Claudia R. Baquet (University of Maryland School of Medicine)

Lower socioeconomic status (SES) has been consistently linked with an increased prevalence of cigarette smoking. Community socioeconomic composition reflects 
more than a grouping of people with similar characteristics, and may provide clues to how the socioeconomic context of whole communities influences cigarette smoking beyond an individual's SES alone. This cross-sectional study used a random sample of adults, aged 18-64, residing in six Maryland jurisdictions: Baltimore City and Caroline, Dorchester, Kent, Prince George's, and Somerset counties. Telephone interviews using Computer Assisted Telephone Interviewing (CATI) were administered to 906 participants between April 2006 and February 2007. These data were linked to community social and economic characteristics from the US Census by zip code. Multilevel logistic regression models were used to estimate odds ratios (OR) for community-level SES and cigarette initiation and current use, and a proportional odds logistic regression model using generalized estimating equations (GEE) was used to estimate the OR for smoking intensity as measured by packs of cigarettes smoked per day. Community-level SES was captured by standardized composite measures. After adjusting for age, race/ ethnicity, gender, individual level education, marital status, and county of residence, the odds of smoking initiation and current smoking were lower among higher SES communities than among lower SES communities. Smoking intensity increased by 5 percent for each unit increase in the low SES composite measure. These results were independent of age, race, gender, individual level education, marital status, and county of residence. Community-level SES was associated with smoking initiation, current use, and smoking intensity. Our results suggest that community-level SES exerts an independent effect on smoking prevalence in communities. Thus smoking cessation policies should target whole communities in addition to individuals within communities.

\section{4-23 Differences In Neighbourhood Food Retail Environments By Socio-Economic Status In Ottawa, Canada}

Elizabeth Kristiansson; Melissa Calhoun; Stephanie Leclair; Sam Herold; Mike Sawada; Moe Garahan; Nazeem Muhajarine; Ian Cross; David Salisbury; Margaurite Keeley; Vivien Runnels; Anne Musiol; Cliff Gazee; Russell Mawby; Amira Ali; Ron Labonte (University of Ottawa)

Food deserts - disadvantaged neighbourhoods characterized by relatively poorer access to healthy and affordable food - may be an important barrier to healthy eating for those who are low income. Such spatial inequalities may help us to understand social disparities in diet and obesity. We present evidence from a new Canadian community-academic collaborative study investigating neighbourhood food environments by levels of neighbourhood advantage. Neighbourhood definition was done iteratively; combining the physical boundaries and functional approaches, real estate maps and our city and community team memberýs knowledge; 89 neighbourhoods were defined. We found no significant differences in the number of grocery stores per thousand, but people in lower income neighbourhoods were significantly closer to grocery stores ( 845 meters) than people in high income neighbourhoods (4500 meters). The lowest SES neighbourhoods had 4 times as many convenience stores per thousand as the highest income neighbourhood and 2 times as many convenience stores as neighbourhoods in the next lowest quintile. The middle and lowest SES neighbourhoods had more fast food outlets per capita than the other neighbourhoods. Fast food outlets were closer to the population weighted centroid (446 metres) in the lowest SES neighbourhoods than in any of the other neighbourhoods $(845,642,1525$, and 2411 meters for quintiles $4,3,2$, and 1 , respectively). $51 \%$ of the schools in Ottawa were within 500 metres 
of a fast food outlet: this figure was $85 \%$ for schools in the lowest income neighbourhoods but only $21 \%$ for schools in the highest income neighbourhoods. Maps and photographs taken by neighbourhood residents and team members will be incorporated in the presentation.

\section{4-24 Training Community Members to Recruit Participants onto Cancer Clinical Trials:}

James Butler, DrPH (University of Pittsburgh Graduate School of Public Health) Innovative health promotion programs have successfully used community members to provide outreach, screening, and educational services. These individuals understand, care about, and are able and willing to work with their community, and others spontaneously seek them out for advice, support, and assistance. Their role is referred to by many names, including lay health advisor and community health educator. However, little research exists on the training of community members for recruiting and retaining participants of cancer clinical trials. We hired and trained 62 residents and managers of public housing developments as Community Outreach Residents (CORE) to recruit public housing residents onto a cancer clinical trial, known as Pathways to Health. Five weeks prior to a participant recruitment health fair, the CORE were provided with an intensive training that highlighted the goal and design of the trial, their responsibilities as CORE team members, and strategies needed for participant recruitment and retention. Researchers and practitioners may consider using the CORE Training Model as a means of engaging the community and as an aid in overcoming barriers to participation in cancer clinical trials by underserved populations.

\section{4-25 The Relationship Between Diabetes Prevalence and Neighbourhood Environments and Resources in Toronto, Canada}

Dr. Rick Glazier; Peter Gozdyra; Maria Creatore; Jonathan Weyman; Anne-Marie Tynan; Dr. Gillian Booth (Centre for Research on Inner City Health, The Keenan Research Centre in the Li Ka Shing Knowledge Institute of St. Michael's Hospital.) Context: Diabetes mellitus is growing in developed countries affecting $8.8 \%$ adults in Ontario, 2005. Clinical, regulatory, environmental and health promotion strategies are needed to promote life styles that reduce obesity, the leading cause of increasing diabetes. Objective: To examine the relationship between environments, resources and diabetes. Design: Ecological study using diabetes rates and numerous local environmental factors. Setting: Toronto's 140 neighbourhoods. Participants: 2.4 million Toronto residents. Instrument: An Activity Friendly Index (AFI) derived using residential density, density and access to retail, car ownership and crime. A Healthy Resources Index (HRI) derived using access to healthy foods, recreational spaces, parks, and family doctors. Main Outcome Measure: The correlation between AFI/HRI and diabetes prevalence rates and daily physical activity. Results: The AFI had a moderate inverse correlation with diabetes rates $(-0.235, \mathrm{p}<0.01)$ and a strong direct correlation with physical activity $(0.597, \mathrm{p}<0.001)$. The HRI had no significant relationship with diabetes rates $(-0.099, \mathrm{p}>0.05)$ but was associated with physical activity $(0.526$, $\mathrm{p}<0.001)$. Conclusions: Neighbourhood characteristics were related to diabetes and physical activity. Although not necessarily causal, these findings suggest that urban planning, zoning, public transit may be avenues for intervention to reduce obesity and diabetes. 


\section{4-26 Spatial Inequalities and Health Disparities: Neighbourhood Resources, Socio-Economic Status and Health Outcomes in Ottawa}

E. Kristiansson; S. Herold; A. Ali; M. Calhoun; S. Leclair; A. Musiol; M. Sawada; R. Labonte; M. Keeley; M. P. Parenteau; R. Mawby; I. Cross; C. Gazee (University of Ottawa)

Contextual research is increasingly important in population health as evidence mounts that the places in which we live can affect both our health and the gap in health outcomes between high and low socio-economic groups. The communityuniversity Ottawa Neighbourhood Study is funded by CIHR and was developed to measure and map features of the neighbourhood landscape that affect health and that are amenable to policy and planning interventions.

Neighbourhood definition was done iteratively; combining the physical boundaries and functional approaches, real estate maps and our city and community team member knowledge; spatial techniques of clustering and wombling were used. Neighbourhoods were aggregated from dissemination area geography; we defined approximately 89 neighbourhoods in Ottawa. Indicators of neighbourhood resources for health were carefully chosen based on their theoretical or empirical link to health and health inequalities. Maslow's hierarchy of needs and socio-ecological theory were conceptual frameworks for indicator development. Indicator data was obtained from multiple sources, including the 2001 Canadian Census, DMTI Enhanced Points of Interest, Elections Canada, the City of Ottawa, the National Capital Commission, and Ottawa Police Services.

We gathered data on indicators of neighbourhood resources for health: the natural environment, goods, services, and amenities (food, recreation, education, health services, financial services, and mobility), the social environment (e.g. social cohesion, crime), the built environment (e.g. housing quality), and neighbourhood socio-economic deprivation. The Ottawa Public Health Department linked neighbourhoods to health outcomes: the rate of admission for ambulatory care sensitive conditions, rate of smoking in pregnancy, and rates of emergency room use. We will present maps indicating the distribution of neighbourhood resources by neighbourhood deprivation level, overlaying this with health outcomes.

\section{4-27 Food Availability, Neighborhood Socioeconomic Status, and Dietary Patterns Among African Americans with Type 2 Diabetes}

Rachel A. Millstein; Hsin-Chieh Yeh; Frederick L. Brancati; Marian Batts-Turner; Tiffany L. Gary (Department of Epidemiology, Johns Hopkins University, Bloomberg School of Public Health)

The goals of this study were to determine associations between neighborhood-level food sources and socioeconomic status (SES) with dietary patterns and body-mass index (BMI). African American adults $(\mathrm{n}=132)$ with type 2 diabetes in Project Sugar 2 (Baltimore City, Maryland) answered the Ammerman dietary assessment which gives a total dietary risk score and subscores for meat, dairy, starches, and added fat. Participants' height and weight were measured using standard methods. Food source availability (food stores, convenience stores, other food stores, restaurants, and other food service places) and SES data from the 2000 US census at the tract-level were linked to individual-level data. Linear mixed effects regression models with random intercepts were used to account for neighborhood clustering and for individual-level SES and potential confounders. The presence of restaurants and other food service places in census tracts were associated with better dietary patterns (added fat subscore adjusted \&\#946; = -1.1, 95\% CI=-1.8, -0.4, and \&\#946;= -1.0, 95\% 
$\mathrm{CI}=-1.7,-0.3$, respectively). The presence of convenience stores and lower neighborhood SES were not significantly associated with worse dietary patterns or BMI, although trends were in the hypothesized direction. These findings provide additional evidence for structural improvements to food environments in urban and low-income African-American neighborhoods.

\section{4-28 Individual Sociodemographics, Food Environment, and Fruit and Vegetable Intake in Urban Adults}

Shannon N. Zenk, PhD; Laurie Lachance, PhD; Amy J. Schulz, PhD; Graciela Mentz, PhD; William Ridella, MPH, MBA (University of Illinois at Chicago)

Background. In this analysis, we examine relationships between multiple aspects of the retail food environment and fruit and vegetable $(\mathrm{fv})$ intake as well as differences by individual sociodemographics (e.g., race/ethnicity, age, income, car ownership), drawing on a sample of 919 African-American, Latino, and White adults in three Detroit communities.

Methods. The Healthy Environments Partnership, a CBPR partnership, conducted the study. We measured $\mathrm{fv}$ intake using a modified Block food frequency questionnaire. Using GIS, direct observation, or respondent self-report, we measured the food environment including street-network distance to the nearest supermarket; neighborhood (half-mile around home) food store availability (large grocery, small grocery, specialty, convenience, liquor); observed neighborhood fresh fv availability, selection, quality, and price; and satisfaction with neighborhood fv options. We used HLM 6.03 to estimate relationships.

Results. Preliminary results show that the relationship between availability of a large grocery store in the neighborhood and $\mathrm{fv}$ intake differed by race/ethnicity. Latinos with a large grocery store in their neighborhood consumed more $f v$ than African-Americans residing in neighborhoods with a large grocery store. We found few other statistically significant associations.

Conclusion. Further research is needed to examine whether these observed discrepancies result from other environmental conditions that differentially support $\mathrm{fv}$ purchasing and consumption.

\section{SOCIAL DETERMINANTS OF HEALTH}

\section{5-01 Modest Social Gradients in Adverse Reproductive Outcomes in Santiago de}

Kaufman JS, Pino P, Alonso FT, Bustos P, Amigo H. (UNC School of Public Health, Department of Epidemiology)

Socioeconomic gradients in adverse birth outcomes such a premature delivery $(<37$ weeks gestation) and low birthweight $(<2500 \mathrm{~g})$ are well established in the US. Santiago is a city of roughly 5 million inhabitants experiencing rapid socioeconomic change, including substantial immigration from rural areas of Chile and surrounding nations. We examined vital statistics for the 32 comunas that make up the metropolitan area of Santiago, from 2001-2004. There were 329,054 live births recorded in this period, of which 321,909 were singleton births with complete data. Educational attainment was categorized into $<12$ yrs $(34.6 \%), 12$ yrs $(36.8 \%)$, and $>12$ yrs $(28.7 \%)$. Overall risk was low compared to other nations in the region: $6.1 \%$ preterm birth (ptb), $4.6 \%$ low birth weight (lbw). For women $>19$ yrs old $(86.4 \%)$, there was a significant advantage for intermediate over low education for both outcomes $(\mathrm{RR}=0.95$ and $0.94, \mathrm{p}<0.003)$, and for high over low education $(\mathrm{RR}=$ 
0.86 and $0.89, \mathrm{p}<0.001)$. Nonetheless, risk differences were very modest between these strata in absolute terms, with risks varying between low and high groups by only $0.9 \%(6.4 \%$ vs $5.5 \%)$ for ptb and $0.5 \%$ for $\mathrm{lbw}(4.7 \%$ vs $4.2 \%)$. In a generalized linear model (binomial distribution, identity link), the risk difference for low vs high education (adjusted for maternal age, sex, ethnicity, marital status and parity) changed little for $\mathrm{ptb}$ and $\mathrm{lbw}(0.8 \% ; \mathrm{p}<0.001)$. Though statistically significant in this large dataset, these education gradients are trivial in comparison with the US. For example, lbw risks for native-born US women vary between comparable age and education groups by roughly $4 \%$ ( $8 \%$ vs $4 \%$ for whites and $14 \%$ vs $10 \%$ for blacks), a nearly 10 -fold larger magnitude of disparity. The relatively equal social distribution of adverse pregnancy outcome risk in Santiago therefore warrants further study to elucidate the basis for this phenomenon.

\section{5-02 Physical Violence among a Prospective Cohort of Injection Drug Users: A Gender-Focused Approach}

Brandon DL Marshall; Evan Wood; Kathy Li; Julio Montaner; Thomas Kerr (British Columbia Centre for Excellence in HIVIAIDS)

Objective: Although dramatically heightened rates of violence have been observed among injection drug users (IDU), little is known about the gender differences associated with violence among IDU.

Methods: We performed an analysis of the factors associated with received violence among participants enrolled in the Vancouver Injection Drug Users' Study during the years 1996-2006 using generalized estimating equations (GEE). The perpetrator and nature of the violent attack were also examined.

Results: Among 1114 individuals, 193 (44\%) females and 332 (50\%) males reported a violent attack during follow-up. In multivariate analyses, younger age, frequent alcohol use, frequent crack use, downtown eastside residency, and requiring help injecting were positively associated with received violence for both genders $($ all $\mathrm{p}<$ $0.05)$. For females, drug dealing $(\mathrm{AOR}=1.43)$, sexual abuse $(\mathrm{AOR}=1.30)$, and binge drug use $(\mathrm{AOR}=1.31)$ were positively associated with received violence, while homelessness ( $\mathrm{AOR}=1.52)$, incarceration $(\mathrm{AOR}=1.50)$, and frequent heroin injection $(\mathrm{AOR}=1.22)$ were significant for males. Men were also more likely to be assaulted with weapons, and experience violence from the police and from strangers (all $p<0.001$ ).

Conclusions: These findings indicate that the predictors of violence among IDU do not vary considerably by gender, although some differences do exist. Violence is largely driven by drug and alcohol use and engagement in the drug market. These findings indicate a need for continued scale-up of addiction treatment and programs that serve victims of violence.

\section{5-03 Intimate Partner Violence and Heterosexual Men's Sexual HIV Risk Behavior in a Major Urban Area}

Victoria Frye; Danielle Ompad; Sara Putnam; Christina Chan; Sandro Galea; David Vlabov (Center for Urban Epidemiologic Studies)

Background: The relationship between intimate partner violence (IPV) victimization and sexual HIV risk among heterosexual women is well-documented. There is less research on perpetration of IPV and sexual HIV transmission risk among urban men. Methods: Using data from structured interviews with 307 heterosexual men recruited via street-intercept methods between 2005 and 2006 in 36 New York City neighborhoods, we assessed the relationship between perpetration of IPV against a main female partner and inconsistent condom use with that same partner, 
controlling for condom use-related and other factors, such as condom use barriers and relationship dominance. Results: Nearly a third of the men surveyed reported using moderate physical violence and 5\% reported using sexual violence against their main female partners. Multivariate logistic regression revealed that men who perpetrated physical IPV were $60 \%$ less likely to report consistent condom use as compared with men who did not use violence, while controlling for sociodemographic, condom use-related and other factors identified as significantly associated with the outcome in bivariate analyses. Conclusions: IPV perpetration by heterosexual men makes a unique contribution to sexual HIV transmission risk behaviors. Given the growing heterosexual HIV epidemic, interventions that simultaneously address men's sexual HIV risk behaviors and IPV are suggested.

\section{5-04 A Practice-based Approach to a Social Health Model}

Pamela Young, PhD, LCSW-C (LifeBridge Health/Sinai Hospital)

This presentation describes a social health model that recognizes a relationship of social and cultural factors to health status. It was developed in an urban hospital as an effort to respond to the barriers that poverty erects to health and well-being for women and their infants. It has grown to a range of psychosocial responses to the consequences of poverty and its accompanying problems such as underemployment, poor education, inadequate housing, violence, and drug addiction.

The social health model extends assistance beyond traditional medical services. Psychosocial interventions focus on social factors that impair overall health and well-being. Services consist of education, counseling, advocacy, and referrals to resources that combat the consequences of poverty. The model operates through the mechanism of an intense supportive relationship of program participants with program staff. Factors from their own lives, such as substance abuse, single motherhood, and violence are aspects of the culture of poverty that heighten staff's sensitivity to program participant's lives, thereby building trust and understanding in the relationship. The presentation will demonstrate the application of this social health model with practice approaches and examples of women's experiences living with HIV, addictions, perinatal mood disorders, and intimate partner abuse.

\section{5-05 Is Work Healthy for Older Poor Adult: The Case of Elderly Males in Three Underprivileged Communities In Beirut.}

M. Chaaya; A. M. Sibai; H. Chemaitelly; Z. El Roueiheb (American University of Beirut)

Introduction: In view of the growing number of older persons, the deteriorating economic conditions, and the lack of pension systems, work among the elderly deserves special attention. This paper examines how work links to depression among Lebanese older adults. Methods. The sample included 328 elderly males living in 3 poor urban communities. Depression was assessed using the 15-item Geriatric Depression Scale (GDS). The exposure variable was working for pay at the time of the survey. Covariates included demographics, health characteristics, financial resources and social capital.

Results. Around one third of the elderly 64 years and above were working. Unadjusted data showed a protective effect of work on depression (OR $=0.28,95 \%$ $\mathrm{CI}=0.12-0.67)$. In the adjusted model the statistical significance disappeared. Health characteristics were significantly associated with depression.

Conclusion. This study is an eye opener on a subject rarely addressed in this area of the world. Old age is viewed as a decline in abilities while in reality many 
older adults are still able and ready to work. Social policies for elderly should address work and not merely pension schemes.

\section{5-06 The Impact of Positive Psychology on Quality of Life Among Aging African American Women in an Urban City}

Candace Parker, PhD (University of Maryland)

Aging African-American women are disproportionately affected by negative health outcomes and mortality. Life stress has strong associations with these health outcomes. The purpose of this research was to understand how aging African American women manage stress. Specifically, the effects of coping, optimism, resilience, and religiousness as it relates to quality of life were examined.

This cross-sectional exploratory study used a self-administered questionnaire and examined quality of life in 182 African-American women who were 65 years of age or older living in senior residential centers in Baltimore using convenience sampling.

Results revealed that the positive psychological factors examined were positively associated with and significant predictors of quality of life. The correlations indicated that of the six coping dimensions measured in this study, planning was the most positively associated with quality of life as well as optimism, resilience, and religiousness were significantly correlated with quality of life.

In the linear regression model, again the coping dimension of planning was the best predictor of quality of life. Optimism, resilience, and religiousness were also significant predictors of quality of life. It appears as if positive psychology plays an important role in improving quality of life among aging African-American women living in urban environments.

\section{5-07 Women's Access to Health Care in Ghana: Effects of Education, Residence, Lineage and Self-Determination}

John Boateng; Constance Flanagan (The Pennsylvania State University)

Women's physical and psychological access to health care was analyzed using the 2003 Ghana Demographic and Health Survey (GDHS), a nationally representative study to monitor population and health in Ghana. Female respondents from the 2133 cases in the couple's data set were used in this study. Women's level of education was positively related to physical but not to psychological access to health care. Residing in an urban area was positively related to both types of access. Matriliny consistently showed positive effects on physical access. In addition to these demographic factors, both physical and psychological access were positively related to women's self-determination, i.e., women's right and ability to make real choices about their lives including their health, fertility, sexuality, childcare and all areas where women are denied autonomy and dignity in their identities as women. Selfdetermination factors both mediated the effects of background factors on access and added explanatory power to the models.

\section{5-08 The Wholeness Index}

Rachael S. Jackson (The J. McDonald Williams Institute)

Research shows that social inequalities and health are intimately linked; regions with less disparity enjoy better overall health. However, typical approaches to measuring disparity suffer from at least one of three major shortcomings. Many rely on data that is sampled from spatial units that are too large to target for change (e.g., counties, cities, etc.). Many are unidimensional in nature, proving far too simple to reflect the complex nature of public health. Those indices that are multidimensional 
often capitalize on census data, updated infrequently, rendering action less than timely.

The Wholeness Index offers a new approach to measuring disparities in wellbeing and health. First, the broad base of indicators included in the index ensures that the complex nature of wellbeing is adequately reflected. Also the Index uses local administrative data, which increases the availability and recency of data. In addition, the spatial methodology powering the Index permits the inclusion of data from different units of analysis and visual support to analysts and policy makers.

Using Dallas, Texas, as a model, we calculated neighborhood-level variance from the city average and combined these to create a wholeness score for each indicator. Indicators were mapped to visualize the hot spots within the city.

\section{5-09 Societal Influences on Birth Practices in Underserved Urban Slum of Indore, India}

Siddharth Agarwal; Karishma Srivastava; Prabhat Jha; Partha Haldar; Rajeev Kumar (Urban Health Resource Centre)

Background: Birth practices are greatly influenced by the traditions and values of the society. People in slums often continue to practice harmful behaviours. It is imperative to understand birth practices in order to devise strategy for improving maternal and neonatal health of urban poor.

Methodology: In 11 slums, 312 mothers of infants (2-4 months) were interviewed on birth practices. Focus group discussions with mothers and birth attendants were employed to explore the reasons behind the practices followed by mothers.

Results: Home births were preferred despite physical proximity of hospitals. Of all deliveries, $72.1 \%$ were conducted at home. Of these, $56.4 \%$ were conducted in slum-homes and $15.7 \%$ in native villages. Of slum-home births, $77.3 \%$ births were conducted by slum-based traditional birth attendants (TBAs) and remaining by family members/neighbours. Only 40.5\% TBAs had received training in the preceding year.

Factors like confidence in TBAs, fear of delivering baby at hospital, economic and transportation constraints and lack of preparedness of family to escort woman to health facility contribute to the choice for home delivery.

Birth practices were influenced by traditions and beliefs, reinforced by motherin-law and TBAs. Mothers feared harm to babies failing community norms. The use of five cleans was very low (less than 50\%). Warming of birth room, appropriately wrapping the newborn and postponing bathing (for $24 \mathrm{hrs}$ ) were practiced in $38 \%$, $54 \%$ and $37.5 \%$ of families. Breastfeeding was initiated within an hour of birth and prelacteals avoided by 48.9 .

Conclusion: Options emerged for improving care includes 1) early pregnancy identification, regular counselling by trained slum-based health volunteers, 2)Involving mother-in-laws, early adopters and community leaders for reinforcing health messages 3)periodic competency-based training of TBAs 4)strengthening community linkage with nearby/affordable health facilities for availing obstetric services.

\section{5-10 Health Literacy: Why is it a Major Urban Public Health Issue?}

Andrew Pleasant, PhD; Christina Zarcadoolas, PhD (Mount Sinai School of Medicine) The U.S. population faces well-recognized health risks including chronic diseases, environmental degradation, and natural and man-made disasters. But there is a silent killer, less diagnosed and essentially untreated, maneuvering just below the surface that poses a great threat to public health in the 21 st century. This silent killer is low health literacy ý the reality that almost half of adults in the U.S., over 90 
million people, struggle to find, understand, and correctly use basic health information. The consequences of low health literacy include inadequate skills to understand health and environmental health risks, to take actions to mitigate these risks, to access and use the health systems, lack of social empowerment and selfefficacy, poor health outcomes, diminished quality of life, and increased financial costs to society. We will present an elaborated model for understanding health literacy. The new model delineates the role of four core domains: fundamental literacy, science literacy, civic literacy and cultural literacy. Examples from health and environmental health messages will be used to demonstrate common problems, and present guidelines for constructing effective outreach, education and translation activities to advance public health literacy. The presentation is based on the new book, Advancing Health Literacy: a Framework for Understanding and Action (Jossey-Bass/Wiley, June 2006, C. Zarcadoolas, A. Pleasant \& DS Greer).

Teaching Goals:

Participants will learn principles of health literacy in the areas of science, civic and cultural literacy that they can apply to improve the daily performance of health professionals and health programs.

Participants will be able to identify some health literacy demands their health specialty or health focus places on low health literate patients/consumers.

\section{5-11 The Association Between Neighborhood Population Decline and Time yo Hiv Seroconversion Among Injection Drug Users (IDUs) in Baltimore}

Arijit Nandi; Haitao Chu; Katharine Poundstone; David Vlahov; Gregory D. Kirk; David D. Celentano; Shruti H. Mehta (Johns Hopkins Bloomberg School of Public Health)

Few studies have attempted to understand how the urban environment influences the risk of HIV transmission among IDUs. As a result of macro-level economic changes, Baltimore City experienced rapid population decline in the latter half of the twentieth century, with the most rapid decrease from 1990-2000. We used data from a community-based cohort of IDUs recruited from 1988-1998 to assess whether neighborhood-level population change from 1990-2000 influenced time to HIV seroconversion. 1,926 HIV-negative participants returning for $>=1$ follow-up visit were geocoded to their neighborhoods of residence. Among 211 neighborhoods, median population decline was $1.0 \%$ per year $(\mathrm{IQR}=0.4 \%, 1.8 \%) .321$ participants seroconverted through 2004. Lognormal survival models with random effects were used; individual-level variables were treated as time-updated covariates. Median time to HIV seroconversion was $101 \%$ longer among participants living in neighborhoods in the lowest quartile relative to the highest quartile of population decline. After adjustment for sociodemographic characteristics and drug-related risk behaviors (i.e., injection frequency, sharing needles, cocaine use), participants living in neighborhoods in the lowest quartile of population decline had 59\% more HIVfree time relative to those in the highest quartile [RT $=1.59,95 \% \mathrm{CI}(1.05,2.40)]$. Public health efforts aimed at reducing HIV transmission among IDUs must address characteristics of the urban environment.

\section{5-12 Gender, Aging, Poverty and Health: How Men and Women Survive and Adapt to Old Age in Nairobi Slums}

Netsayi Noris Mudege (African Population and Health Research Center)

This paper uses qualitative data, from two Nairobi slums, to discuss how division between the domestic sphere, defined as a predominantly female domain, and the 
public sphere, defined as a male domain, impacts on survival during, and adaptation to old age. Although this paper adopts some of the tenets of the life cycle approach, it posits that sometimes women's participation in the domestic sphere gives them a gender advantage over men in terms of health, and adaptation to old age. The paper will discuss the impact of gender roles in earlier life on the cultivation of social networks and how these networks in turn impact on health as well as social adjustment in old age. It will also investigate how the aged have adjusted to new demands they experience as a result of high morbidity and mortality rates of adults in reproductive age groups. Lastly the paper will examine links between health and poverty among the old, and offer policy prescriptions. To define old age this paper uses chronological, functional and social cultural markers. Although the age of 55 will be used to define old age, the definition also includes other qualifiers such as changes in social roles and capability.

\section{5-13 Perceptions of Neighborhoods and Salivary Cortisol Levels in the Baltimore Memory Study}

B. K. Lee; T. A. Glass; M. J. McAtee; B. D. James; B. S. Schwartz (Johns Hopkins Bloomberg School of Public Health)

Studies suggest that perceptions of physical and social conditions of urban communities are associated with psychosocial stress, which may be one mechanism through which neighborhood conditions influence health status. However, studies using subjective ratings of both neighborhoods and stress may suffer from samesource bias. We examined whether subjective ratings of neighborhoods were associated with cortisol levels in a cross-sectional analysis of 967 men and women aged 50-70 years sampled from 65 neighborhoods. Four salivary cortisol samples across one study visit were used to create seven summary metrics that objectively measured HPA axis function. Perceptions of neighborhoods were assessed by questionnaire and three separate scales were created to measure general neighborhood problems, social cohesion, and social and physical disorder. Using multiple linear regression to adjust for age, sex, race/ethnicity, wealth, and time of day, we found no consistent evidence that any of the three neighborhood perception scales were associated with the cortisol metrics. These results underscore potential difficulties in using subjective measures in neighborhood studies. To the extent that the cortisol metrics are valid measures of HPA axis dysregulation, the findings do not support the conceptual model that perceptions of neighborhoods are associated with health outcomes through psychosocial stress pathways.

\section{CITIES AND INFECTIOUS DISEASES}

06-01 Inter-City and Intra-City Collaborative Models in TB Contol and Service Delivery: Lessons from the Pilot Site in Quezon City, Philippines

Loyd Brendan P. Norella, MD, MPHM (Health Policy Development Program UP Diliman, Quezon City, Philippines) (Health Policy Development Program)

Tuberculosis is a major public health problem in the Philippines. The World Health Organization (WHO) 2005 Report ranks the Philippines as 9th among the 22 high TB burden countries. In 2003, the mortality rate of TB was estimated at 49/100,000 per year, translating to 114 deaths per day.

In response to the growing TB problem, the National TB Program (NTP) has adopted the internationally recommended DOTS program (Directly Observed 
Treatment Short Course) in 1996. Under the leadership of the Department of Health $(\mathrm{DOH})$ and the Philippine Coalition Against Tuberculosis (PhilCAT), the country attained the global program targets of success rate of $80 \%$ and case detection rate of $70 \%$ in 2005. In spite of the many achievements of the NTP in improving the country's TB response, a lot of effort is still needed to achieve the country's vision of reducing TB mortality and prevalence by half in 2010 .

To further improve the national response to $\mathrm{TB}$, the $\mathrm{DOH}$ adopted a localized and zonal approach to TB control. Guidelines were drafted to enhance the capability of local governments to collaborate with all sectors and with each other. This was in terms of effectively managing the local implementation of the TB control program. In 2005-2006 the guidelines were implemented as a pilot in Quezon City with full support of the City Health Office. A recent review of the outcomes of the program in the site, however, identified areas of weakness. There were concerns in the areas of resource management, referral systems, monitoring and evaluation and sustainability. In short, the planned intra-city and inter-city collaborative mechanisms were not maximized due to several factors identified. This study will discuss the factors in detail and provide suggestions for improvement. Lessons from this experience may help guide other locales in designing similar yet improved programs to facilitate DOTS delivery among city-based underprivileged and highly mobile patients.

\section{6-02 Prevalence and Correlates of M. Tuberculosis Infection Among Injection Drug Users at Risk for HIV in Tijuana, Mexico}

Richard S. Garfein, PhD; Timothy C. Rodwell, MD, PhD; Kimberly C. Brouwer, PhD; Antonino Catanzaro, MD; Rafael Laniado-Laborin, MD; MPH; Minya Pu, MA; Remedios Lozada, MD; Andrea Mantsios, MS; Peter G. Chiles, BA; Robert Deiss, MD; Steffanie A. Strathdee, (University of California, San Diego, School of Medicine)

Background: Recent increases in substance abuse and HIV prevalence in Mexican cities bordering the U.S., where tuberculosis (TB) is endemic, highlight the need for estimates of latent $\mathrm{TB}$ infection for predicting the future health needs of injection drug users (IDUs) and other populations at risk for HIV/TB co-infection.

Methods: Subjects included current IDUs, age $>18$ years, recruited using respondentdriven sampling (RDS) for an ongoing IDU cohort study in Tijuana, Mexico. After informed consent, subjects underwent risk factor interviews, pre-test counseling, venipuncture for HIV antibody testing and TB screening using an in vitro QuantiFERON-TB Gold (QFT) assay, which is insensitive to Bacillus CalmetteGuerin (BCG) vaccination. HIV+ subjects and those reporting symptoms suggestive of active TB were referred for medical care. Independent associations between QFT status and subject characteristics were detected using RDS-adjusted multivariate logistic regression.

Results: The 973 subjects enrolled were, on average, 36 years old (IQR 3142 years), $89 \%$ male and $98 \%$ Mexican born. Most (78\%) had traveled to the U.S., $97 \%$ mostly injected heroin, and 77\% had BCG scars. Overall, 70\% tested QFT+ and $4.5 \% \mathrm{HIV}+$. Of these $652 \mathrm{QFT}+$ subjects, $13(2.0 \%)$ had symptoms suggesting active TB and 1 was HIV+. QFT positivity was independently associated with injecting drugs in the past 6 months outside one's own home $(\mathrm{OR}=1.49 ; 95 \% \mathrm{CI}$, 1.12-2.0), knowing $>100$ IDUs $(\mathrm{OR}=1.43 ; 95 \% \mathrm{CI}, 1.08-1.90)$, injecting $>5$ years $(\mathrm{OR}=1.45$; 95\% CI, 1.01-2.10), being born in Mexico $(\mathrm{OR}=4.46$; 95\% CI, $1.68-$ 11.88), always lived in Tijuana ( $\mathrm{OR}=1.44 ; 95 \% \mathrm{CI}, 1.02-2.03)$, and enrolled at sites closer to the U.S. border $(\mathrm{OR}=1.54,95 \% \mathrm{CI}, 1.00-2.38)$. 
Conclusions: Proximity to the U.S. border and degree of interaction with other IDUs were associated with TB infection. Interventions are urgently needed to prevent reactivation of latent $\mathrm{TB}$ infections among IDUs who are at high risk for HIV infection.

06-03 Study of Designated Microscopy Centers (DMCs) with Level of Health Care Services for Case Detection Under Revised National Tuberculosis Control Programme (RNTCP) at Pimpri Chinchwad Municipal Corporation (PCMC) Urban area, Pune, India. Dr. Kishor Khillare; Dr. Rajshekhar Iyer (Pimpri Chinchwad Municipal Corporation) Objectives

To identify correlation between level of health care services and case detection at its DMCs.

To know contribution of referrals for case detection by Private Medical Practitioners (PMPs)

To find areas of improvement to increase case detection.

Methodology

Total 15 DMCs with its health care level institutes studied from January to December 2006 using Laboratory, TB and Chest symptomatic referral registers with Referral letters from PMPs.

Simple statistical tools like Average, Ratio, Percentage and Co-relation method used for study.

Observations

In PCMC urban area RNTCP achieved high performance indicator with an annual New Sputum Positive (NSP) case detection rate 78\% (more than the national target of $70 \%$ )

DMCs with Tertiary Health Care level institute detected NSP 32.3\%, New Sputum Negative (NSN) 35\% and high New Extra Pulmonary (NEP) cases $56.5 \%$.

DMCs with Secondary Health Care detected NEP cases $36.4 \%$ with high NSP 45.9\% and NSN 52.9\% cases where PMPs have contributed more.

DMCs with Primary Health Care detected less NSP 20.8\%, NSN 12.1\% and NEP $7.1 \%$.

PMPs contributed $23 \%$ of total NSP cases while field health workers (ANM/ MPW) shared less than $2 \%$ of total NSP case detection.

Conclusions

DMCs should be strengthened with diagnostic and supportive health care services in relation to total lung health care for better case detection.

Case detection can be increased with involvement of PMPs.

Public health care institutes with resources limiting set-up may improve case detection by providing outreach services and participation of field health workers with relation to identifying chest symptomatic and referring them to nearest DMC may enhance case detection.

06-04 Assessing the Effectiveness of Directly Observed Therapy Short Course (DOTS) in the Treatment of Tuberculosis in Igboora Community

F. O. NWAOHA; O. O. OGUNAIKE; O. T. ADETONA (ACTION GROUP ON ADOLESCENT HEALTH(AGAH))

OBJECTIVE

This study was carried out to assess the effectiveness of DOTS in the management of patients with tuberculosis over a ten year period. The study compared the cure-rate, 
default rate, relapse-rate, treatment completion and failure and fatality rates of the preDOTS era (1983-1993) with DOTS era (1993 - 2003)

\section{METHOD}

This was a clinic-based retrospective comparative study done in Igbo-ora. The target populations were patients treated for tuberculosis between 1983 and 1993 before inception of DOTS and those who were treated between 1993 - 2003 under the DOTS system.

A total number of 633 patients treated during this periods were studied and the treatment outcome; as shown below were analyzed and compared.

\section{RESULTS}

Mean age prevalence was 32.9 yrs (SD-14.3) and 31.7 yrs (SD-20.8) for DOTS and pre-DOTS respectively. Pulmonary-tuberculosis accounted for $96 \%$ of all cases in the era.

Out of the patients treated $412(65.1 \%)$ were for DOTS and $221(34.9 \%)$ were treated prior to DOTS.

Using DOTS regime, cure-rate was 289 (70.1\%) compared with 24(10.9\%) for pre-DOTS $(\mathrm{X} 2=202.256 ; \mathrm{df}=1 ; \mathrm{P}<0.0001)$ treatment completion for DOTS was $361(87.6 \%)$ compared with $38(17.2 \%)$ for pre-DOTS $(\mathrm{X} 2=306.18 ; \mathrm{df}=1 ; \mathrm{P}<$ $0.0001)$ those that defaulted for DOTS were 27 (6.6\%) compared with $176(79.6 \%)$ for pre-DOTS (X2=352.68; df $1 ; \mathrm{P}<0.0001)$. Mortality was however observed to be higher in DOTS $26(6.3 \%)$ compared with $4(1.80 \%)$ for pre-DOTS.

CONCLUSION

The cure/treatment completion rate of TB in the DOTS era was higher than the pre-DOTS era while the default rate was lower in the DOTS than in the pre-DOTS era.

Mortality-rate was observed to be higher in DOTS than in pre-DOTS and this could be as a result of better record keeping and monitoring system.

\section{6-05 Correlates of Self Efficacy for Condom Use with Steady Versus Casual Partners Among High-Risk Non-Injection Drug Using Women in New York City}

Sara Putnam; Guozhen Xu; Princess Fortin; Sebastian Bonner; Debbie Lucy; Beryl Koblin (Center for Urban Epidemiologic Studies)

Urban drug-using women are at increased risk of sexual transmission of HIV, and understanding barriers and facilitators of condom use is a priority. Though research shows that condom use varies by sexual partner type and that self-efficacy for condom use is a determinant of condom use behavior, little is known about how self efficacy for condom use varies by partner type. In spring 2006, 403 sexually active, non-injection drug using HIV-negative women living in New York City completed a baseline questionnaire for a vaccine trial knowledge and sexual risk reduction counseling intervention. Self-efficacy for condom use was ascertained separately for casual and steady partners. Bivariate analyses indicated that compared to women with high self efficacy for condom use with steady partners, those with low selfefficacy were more likely to be younger, Latina, and to report recent use of barbiturates or Valium as well as current or past experience of emotional, physical or sexual violence. Correlates for low self-efficacy for condom use with casual partners included unemployment, overnight stay in a shelter or welfare residence in the last 12 months, less acceptance of the Western biomedical approach to illness, and recent marijuana use. Poor condom communication, current depression, and negative outcome expectancies for condom use were associated with low self efficacy for condom use for both steady and casual partners. Self-efficacy for condom use with steady partners appears to be heavily influenced by power dynamics within the 
relationship, whereas self-efficacy with casual partners may be more affected by other personal and structural influences such as housing. These factors should be considered in the design of interventions.

\section{6-06 Male Circumcision as a Preventive Measure to Combat HIV Infection in Sub Saharan Africa}

Kingsley C. Okonkwo, MD; Babatunde O. Alao, MD (West African AIDS Group) BACKGROUND: At of the end of 2006 there were over 35 million people living with HIV worldwide with over 25 million living in sub-Saharan Africa. Three randomized clinical trails in Africa were discontinued prematurely as evidence accumulated that male circumcision [MC] was superior to no circumcision in preventing HIV. This study evaluates the public health impact of these findings and its application as a tool for promotion of MC as a means of preventing HIV infection in Africa.

METHOD: A Meta analysis and systematic review of literature was used to analyze studies that evaluated MC as risk factor for HIV infection in African men. We evaluated the public health impact including cost effectiveness of MC in prevention of HIV transmission.

RESULTS: This review showed there is reduced risk of HIV infection in men who are circumcised; this association is stronger among men with a higher risk for HIV. Data available provided evidence that the risk of female to male transmission can be reduced by more than $60 \%$.Men seemed to benefit more than women. In some studies when adjusted for confounding factors the association was stronger. The pathophysiology underlying this protection was evaluated

CONCLUSION: There is overwhelming evidence that male circumcision significantly reduces the risk of HIV infection among African men. Studies also show that MC could be highly cost-effective. Provision of large scale MC services as part of HIV preventionprograms in African countries could lead to significant reduction in HIV transmission

\section{6-07 Reporting Rate and Occupational Exposure to Sharp Injuries among Jordanian Healthcare Workers}

Zeinab M. Hassan, RN, PhD; Moayad A. Wahsheh, PhD (Hashemite University)

Objectives: a) determine the effects of the demographic variables such as: age, gender, agency, education level, occupation, experience years, working area, and shift on the number of exposure to sharp injuries among Jordanians healthcare workers; and b) determine the effects of the demographic variables on the reporting rate of sharp injuries.

Method: Cross sectional design was used. Data were collected using a survey that was developed by the Centers for Disease Control and Prevention.

Participants: Two thousands surveys were distributed to healthcare workers who are working in private and public hospitals

Results: One thousand and sixty eight healthcare workers filled out surveys on the number of exposure, and reporting of sharp injuries. Significant effects were found between exposure to sharp injuries and the following variables: education level, working area, and the shift. One third of sharp injuries was occurred in intensive care units $(\mathrm{n}=$ $347,34.5 \%$ ), furthermore, fifty percent of sharp injuries was occurred between 11 pm7 am $(n=500,50.8 \%)$. Reporting rate was very low, which ranged from $9 \%$ to $25 \%$.

Conclusion: Underreporting of occupational exposures to sharp injuries is a significant problem facing Jordanian healthcare workers, which put them at serious risk of infection from blood- borne pathogens. 


\section{6-08 Prevalence of Intestinal Parasites in HIV Seropositive (HIV+) Subjects and High Risk Group (HRG) for HIV Infection in Urban Areas of Nepal}

Nirajan Ghimire (Nepal Academic Development and Community Health Academy) Objectives: This study was carried out to find out the prevalence of intestinal parasites in HIV sero positive (HIV+) subjects and high risk group (HRG) for HIV infection in urban areas of Nepal.

Methods: 150 individuals were enrolled in the study. 104 were HIV+, dwelling at different rehabilitation centers of Kathmandu and 46 were HRG subjects (commercial sex workers and highway truck drivers). Single stool samples were collected along with the questionnaire.

Results: The overall prevalence of parasitic infection was found to be $35.7 \%$ (26.7\% among HIV+ subjects and $47.6 \%$ among HRG subjects). Of the total, 64.0\% (96) were males and $36 \%(54)$ were females. The prevalence of parasitic infection among males and females were $33.9 \%$ and $37.7 \%$ respectively. The prevalence of intestinal parasitic infection was significantly higher among HRG males and females than in HIV+ males and females. Multi parasitic infections were relatively more common among HIV+ subjects $(7.5 \%)$ in comparison to HRG subjects $(5.1 \%)$. Similarly the protozoan infections were relatively higher in HIV+ subjects $(4.9 \%)$ compared with HRG subjects $(1.7 \%)$.

Conclusions: Over one third of the study subjects were infected with different intestinal parasites indicating the need of health education, provision of toilet facilities and safe drinking water in urban areas.

\section{6-09 Heterosexuals at High Risk of HIV Acquisition in Washington, D.C.: Sexual Behavior Differences Between Urban Neighborhoods}

Manya Magnus (The George Washington University School of Public Health and Health Services, Department of Epidemiology and Biostatistics, Washington, D.C.); Irene Kuo (The George Washington University School of Public Health and Health Services, Departmen (GWU/School of Public Health and Health Services, Department of Epidemiology and Biostatistics)

Background: Washington, DC, has the highest AIDS case rate in the US, yet little is known about the differences between behavior in geographic areas in the city designated as higher vs. lower risk for HIV.

Methods: Non-seed data collected using respondent driven sampling for the CDC National HIV Behavioral Surveillance system between January-May 2007 were assessed. Adjusted and unadjusted preliminary associations between neighborhoods with differential AIDS and poverty rates using the CDCýs high-risk index were analyzed, using uni-, bi-, and multivariable methods in Stata Version 9.0se.

Results: Of 305 participants, 95\% were African American, 60\% female, mean age 37.9 yrs (sd 10.0; 18-50), and 78.3\% lived in designated high-risk areas. There was substantial sexual risk behavior taking, with mean 6.3 (sd 22.7;0-300) sex partners in the last 12 months; $74.1 \%$ reported no condom use at last heterosexual sex. Mean number of sex partners in higher risk neighborhoods was higher, although not statistically significant [6.6 (sd 25.7) vs. 4.2 (sd 7.3), p=0.19]; there were no other differences in demographic or sexual behavior characteristics between higher-and lower-risk areas.

Conclusion: This study suggests approaches to harm reduction and prevention activities in Washington, D.C. as well as specific ways to target unique urban neighborhoods. 


\section{ENVIRONMENTAL HEALTH}

\section{7-01 World Trade Center Health Registry (WTCHR) Mortality and Cancer Studies Methods \\ James Cone; Gracie Han; Mark Farfel; Robert Brackbill; Lorna Thorpe (New York City Department of Health and Mental Hygiene)}

The World Trade Center Health Registry was developed as a joint project of the new York City Department of Health and Mental Hygiene and the Agency for Toxic Substances and Disease Registry to systematically evaluate the long-term physical and mental health consequences of the 9/11 disaster. During 2003-04, 71,437 persons completed a baseline health survey, including rescue, recovery, and clean-up workers; building occupants, passers-by, residents and children attending schools near the WTC site.

To assess the long-term impact of the 9/11 disaster, mortality of registrants will be ascertained by matching with the New York City Vital Statistics Master Death File and the National Death Index, on a periodic basis. Cancer incidence will be ascertained by matching with New York State Cancer Registry as well as cancer registries of other states with the largest numbers of registrants.

Directly standardized total and cause-specific mortality/incidence rates for the WTCHR cohort will be calculated, using life-table analysis system developed by the National Institute for Occupational Safety and Health. Differential rates across strata of interest (age, gender, worker/residents categories) will be compared using Poisson regression. Nested case-controls studies will be performed and the relationship between exposures and risk factors of interest and outcomes will be assessed by a Cox proportional hazard model, adjusting for confounders.

We will present the proposed methods and projected statistical power of the mortality and cancer studies currently underway.

07-02 Creosote and CCA Timbers in Urban Community Gardens: A CommunityUniversity Partnership Study Examining Soil Contamination and a Strategy For Remediation

Alicia J. Fraser; Wendy Heiger-Bernays; Valerie Burns; Michael D. McClean; H. Patricia Hynes (Boston University School of Public Health)

Since the 1970s, a broad-based community gardening movement has arisen in the US, producing urban agricultural sites in hundreds of cities. A growing body of research has demonstrated the significant contribution of these gardens to the livability of cities by providing nutritious and affordable food; supporting psychological and physiological health; promoting social cohesion, crime prevention, recreation and youth development. The common use of materials such as creosote railroad timbers and CCA lumber has led to concern over soil contamination. In this university-community research collaborative, we describe the nature and extent of Boston community garden soil contamination by polycyclic aromatic hydrocarbons (PAHs) and arsenic. Contaminant concentrations were elevated in soil sampled near timbers and declined with distance from the timbers. We provide details on the development of a remediation plan which calls for removal of timbers and some soil, along with addition of clean city compost. This is the first study to characterize $\mathrm{PAH}$ and arsenic contamination in urban community garden soil and to develop a sustainable remediation strategy. The findings and collaborative methods should provide guidance to other cities and organizations striving to achieve the benefits of urban horticulture. 


\section{7-03 Cleft Defects and Urban - Rural Exposures: the Texas Experience}

\section{C. Messer; T. Luben; P. Mendola; P. Langlois (U.S. EPA / NHEERL)}

Cleft palate and cleft lip, with or without palate (cleft lip + / - palate), are two congenital oral defects that affect children worldwide. Prevalences range from 2.8 to 13.5 for cleft palate and from 4.8 to 17.5 per 10,000 live births for cleft lip +/palate in the United States. Risk factors for cleft defects can be characterized as genetic, maternal and infant characteristics, but many of these qualities are not modifiable. Relatively few studies have explored modifiable environmental risk factors for oral cleft defects. Using Texas Birth Defects Registry and birth records data (1999-2003), we have estimated Poisson and multilevel logistic regression models, to assess the relationship between maternal place of residence (on multiple urban - rural continua) and cleft defects. In models adjusted for infant (sex, plurality, parity, gestational age) and maternal (age, race / ethnicity, education) covariates, we found residence in less urban areas associated with significantly increased risk of cleft lip +/- palate, but not for cleft palate. The association observed for cleft lip was robust across multiple urban - rural exposure definitions. Potential mechanisms, including those associated with pesticides, water, and air quality, will be discussed.

\section{7-04 Impact of Change in the Urban Environment on Emerging Infectious Diseases: An Epidemiological Approach}

M. Rajasekhar; N. V. Nandakumar (S.V. University)

Goal and Scope : Change in the urban environment is an emerging threat to global public health. Hence an epidemiological study on infectious diseases was carried out in the biggest pilgrim town of south India, Tirupati, to see the correlation between disease, environment and climate. Tirupati horizons are being extended as it draws on an average 60000 pilgrims per day. As a result new community relocations are emerging in reserve forest areas. This prompted me to see the association of environmental factors and diseases like Enteric fevers, pyrexia due to unknown origin (PUO), Hepatitis A\&E, Diarrhoea and Malaria.

Method: The morbidity and mortality data were taken from S.V.R.R Govt. hospital. Meteorological data were obtained from the meteorological department of Tirupati The effect of different factors like pilgrim influx, environmental factors, wind velocity, temperature, relative humidity and rainy days on reported hospital admissions have been examined using Poisson regression analysis.

Results: The incidence for infectious diseases was investigated for four years from 2003 to 2006. Pilgrim influx showed positive correlation with diarrhoea, malaria and POUs. Temperature ,Rainy days and Relative humidity also showed positive correlation with the above said diseases. Environmental conditions like waste water collecting pits and open defecation were observed as the prominent environmental factors for occurrence of diseases.

Recommendations: Remedial measures were suggested to the Municipal authorities of Tirupati to take appropriate steps to prevent the diseases.

\section{7-05 The Importance of Place in Driving Urban Health and Environmental Health Disparities}

Sacoby Wilson; Malo Hutson (University of Michigan)

Our paper is a theoretical analysis of the macro-level social, political, and economic structural factors that have produced unequal development within America's metropolitan areas resulting in urban and environmental health disparities along racial and economic lines. This has adverse health consequences for those residents living in poorer 
urban and suburban areas because they are exposed to higher levels of environmental pollutants and have limited opportunities to gain a quality education, access to health care, and increase their economic opportunity. In order to discuss the specific implications of macro-level factors that has contributed to the unequal distribution of resources within U.S. metropolitan areas, we provide a holistic framework which takes an ecological systems approach to understand the affects of urban health and health disparities.

Finally, we contend that in order to improve the urban and environmental health conditions for the most vulnerable urban populations, it will require comprehensive community and regional focused strategies that link local community development efforts to larger macro-level metropolitan regional strategies. These comprehensive strategies must focus on more equitable regional development by improving planning and zoning so that transportation systems, health care, education, and economic opportunities are shared across all communities throughout the metropolitan region.

07-06 Indoor Environment and Asthma Linkage in a South Asian Urban Poor Setting N. K. Dogra; V. Shandilya (The Energy and Resources Institute (TERI))

INTRODUCTION: A community-based case control study was conducted to explore the linkage between the indoor environment (domestic fuel and passive smoking) in relation to urban poor school-going children.

METHODOLOGY: The study subjects comprised of 232 middle school children in the neighborhood. A screening tool for asthma was administered to the children. Parents were administered questionnaires assessing passive smoke exposure, domestic fuel use liquefied petroleum gas (LPG) versus non LPG or mixed fuel use] and possible confounders.

RESULTS: In the neighborhood the unadjusted odds ratio (OR) for the association between passive smoking and asthma was found to be 1.84 [95\% Confidence Interval (CI) 1.06-3.21]. After adjusting for sex of the child, proximity to highway, socioeconomic status of family and housing type, OR was found to be 1.58 (95\% CI 0.87-2.86). If only the slums in the neighborhood are considered, the unadjusted OR for the association between passive smoking and asthma was found to be 2.30 (95\% CI 1.07-4.95) whereas adjusted OR is 1.80 (95\% CI 0.78-4.15). Similarly for domestic fuel use the corresponding unadjusted values for the neighborhood were OR 0.91 (95\% CI 0.54-1.54) whereas adjusted values were OR 0.79 (95\% CI 0.45-1.41). For slums alone unadjusted values were OR 1.05 (95\% CI 0.50-2.19) whereas adjusted values were OR 1.00 (95\% CI 0.45-2.23).

CONCLUSION: The findings demonstrate inconclusive results (after adjusting for possible confounders) with regard to the linkage between the indoor environment and asthma in developing country urban poor settings such as Delhi. Several other studies from developing countries have also shown conflicting results. Further studies with quantitative markers of exposure for domestic fuels and passive smoking are needed. Other methodological issues such as extent of LPG use in households with mixed fuel use and possible exposure misclassification for passive smoking also need urgent consideration.

\section{7-07 Environmental Pollution and Human Exposure to Electromagnetic Fields: A Case Study}

Adilza Condessa Dode; Mýnica Maria Diniz Leýo; Waleska Teixeira Caiaffa (FEDERAL UNIVERSITY OF MINAS GERAIS STATE, BRAZIL-UNIVERSIDADE FEDERAL DE MINAS GERAIS-UFMG)

The electromagnetic fields (EMF) and their effects on human beings and on environment have been addressed to the public health field in Brazil and worldwide, 
recently. Especial concerns have been pointed to the mobile phones regarding users and the communities nearby the Base Stations (RBS).

In Brazil, the number of mobile phone users is estimated in 100 million or more out of 2.3 billion spread all over the world. To ensure the covering of the mobile phones in this large country, a broad network of RBS is needed. Contrasting to a parallel fast growing of RBS spreading in the country a slow pace in the scientific research on the possible health adverse effects has been observed. Moreover, the expanded use has brought concerns to the scientific community as well as the population, in general.

We proposed to present:

I The state of art on possible biological effects resulting from the exposure to radiofrequency (RF) in human beings;

II The methodology developed in order to evaluate the levels of human exposure to the EMF summing up 900 RBS;

III Geocode all RBS in the Belo Horizonte City, concerning the A, B, C and D bands, until 2006;

IV To describe a specific study on EMF resulting from two mobile phones RBS in the city including an environmental perception in the RBS SITE BH-20 neighborhood.

Preliminary results indicated that people living in this RBS surroundings are worried about possible effects on health, environment and reported several pivotal adverse health events related to the exposure. Estimated radiated electromagnetic field and comparisons with the observed measurements carried out in the field, as well as comparisons with the exposure limits recommended by either the national and international guidelines are urgent in urban settings especially when both RBS are beyond the limits recommended by guidelines, i.e., one at 15 meters, RBS called SITE BH-20 and the other at 10 meters, RBS called SITE CT-16, at the maximum gain of the transmitting antennas.

\section{HEALTH INFORMATION TECHNOLOGY AND BEHAVIOR CHANGE}

\section{8-01 Using Technology and Online Collaboration to Enhance Training of After School Program Leaders in Nutrition and Physical Activity}

Sonya Irish-Hauser, MS; Jeanne Goldberg, PhD; Parke Wilde, PhD; Marina Bers, PhD; Lori Ioannone, BS; Christina Economos, PhD. (Friedman School of Nutrition Science and Policy, Tufts University)

BACKGROUND: Gradual shifts toward poor diets and decreased physical activity have been implicated as strong contributors to the childhood obesity epidemic in the US, initiatives to reverse these trends are both prudent and necessary. Interventions aimed at improving nutrition and increasing physical activity in after school environments have shown promise, but leader training requires substantial time and resources.

OBJECTIVE: To compare the efficacy and efficiency of different forms of webbased training and face-to-face training for after school program leaders using a nutrition and physical activity program for children.

METHODS: For 16 weeks, 100 after school programs, nationwide, received one of three training dissemination models: a face-to-face, standard online, or collaborative online. Each program leader completed training modules and 
administered nutrition/physical activity lessons in their after school programs. Multiple measures of program success, including adherence, knowledge, behavior, and attitude change were assessed. Cost per unit of outcome for each success measure was also quantified.

IMPLICATIONS: The results of this study will inform technology-based program dissemination methods, improve cost and time efficiency, increase program flexibility and adaptability, and broaden the scale on which this and other similar nutrition and physical activity programs can be disseminated.

\section{8-02 Minas Telecardio: Telecardiology in the Public Health System of Minas Gerais State, Brazil.}

Clareci Silva Cardoso; Waleska Teixeira Caiaffa; Jussara Teixeira Silva; Maria Beatriz Moreira Alkmim; Galeno Ribeiro; Antonio Luiz Pinho Ribeiro (Federal University of Minas Gerais State)

Cardiovascular diseases are the major cause of morbimortality in Brazil, with high social costs. The long time taken to recognize the symptoms and to look for medical care is responsible for a high rate of ante-hospital mortality. The objective of this study was to implement the telecardiology technology and carry out a clinicepidemiological study investigating the determinants of cardiovascular diseases and the clinical course after intervention. The telecardiology system has been implemented in 82 towns of Minas Gerais State which received a digital electrocardiographer and a computer with internet access. The eligibility criteria for the towns were: population $<10.500$ people and HFP (Family Health Program) coverage $>70 \%$. The study is subdivided into three phases: 1) Construction of a baseline information: health diagnosis of the cities and health workers' satisfaction with the service; 2) Implementation and follow up: periodic training courses and evaluation of the process; 3) Effectiveness evaluation: pre and post intervention comparative studies, including Acute Coronary Syndrome (SCA) patient's follow up with the following events: evolution, quality of life and satisfaction with care. About 253 health workers were trained during a 10 month implementation of the study offering on call activities on cardiology. By April 2007, a total number of 12,125 ECG were performed and around 1,189 patients were eligible for the follow-up study. Complete follow-up was done among 400 patients. Project evaluation after six months pointed $70 \%$ of decrease in referral patients to another cardiac center. Health workers reported their satisfactions related to an opportunity of early diagnosis and full clinical cases discussion.

\section{8-03 Patient and Provider Satisfaction with Health Care to Cardiovascular Emergencies in Brazil: CARDIOSATIS Scales}

Clareci Silva Cardoso; Waleska Teixeira Caiaffa; Marina Bandeira; Graziella Lage Oliveira; Antonio Luiz Pinho Ribeiro (Federal University of Minas Gerais State)

Degree of satisfaction is considered an important measure of quality care as well as an important predictor of the adhesion to the treatment and adequate use of health services. The objective of this study was to construct the scales for evaluation of providers' and patients' satisfaction with the care given to cardiovascular diseases by a distance telecardiology project. Degree of satisfaction is a component of the project effectiveness, which has been implemented in 82 towns of Minas Gerais State. The construction of the scales followed the international standards for development on instruments, including: 1) discussion with specialists on implementation of technologies, including physicians from several specialties and focal 
groups with cardiologists, psychologists and patients. 2) pretest with a group of specialists; 3) pilot study with health workers and patients from ten towns; 4) field study and, 5) evaluation of psychometric qualities of the scales. The focal groups and discussion with specialists had delimited the areas to be enclosed in the instruments and the elaboration of the questions. A total of 20 items comprise each scale. Satisfaction was measured through a five-point likert scale. It also included open questions. The scales information comprised satisfaction with the physical structure, human resources, capacity of resolution, attention and care offered by the service as well as the satisfaction with the received or given care. The pilot study has suggested important changes in the instruments. CARDIOSATIS Scales have showed to be an easy and understandable instrument will accepted by providers and patients. A total of 150 and 400 interviews respectively with workers and patients was carried out. Preliminary results pointed the existence of three factors for Cardiosatis-provider scale. Studies of validity and reliability are still being done.

\section{8-04 Integrative Community Ecologies in Ehealth: Process-Oriented Tailoring in Targeting Lower Income Urban Populations}

Jennifer R. Warren, Ph.D (University of Minnesota)

Cultural tailoring is a strategy in designing health information targeting underserved populations. Sensitivity to culture and incorporating cultural beliefs is effective in health behavior change. Therefore, the use cultural tailoring has migrated online. However, cultural tailoring in ehealth interventions can be reductive to communities and exacerbate health disparities.

Process-oriented community tailoring can overcome reliance upon on unidimensional representations and reduce ehealth inequities. Based on several years of research, my findings indicate culture/cultural identity is not always salient for groups, especially as it interacts with a lower income socioeconomic status. Additionally, group membership identification can fluctuate according to the health issue and prior healthcare experiences in attending to that issue. Group memberships are also multiple and interpenetrated. Findings indicate they should be represented relationally in tailoring. Further, my research shows important relationships between group membership and one's confidence in using the internet for health.

This presentation discusses these significant findings and risks of unidimensional tailoring in ehealth. It will draw on previous studies to advance the Model of Integrative Community Ecologies in Ehealth (MICEE) in process-oriented tailoring in ehealth behavior change interventions, as well as address the health policy, healthcare, and community-based implications in adopting MICEE targeting lower income urban groups.

\section{8-05 Efficacy of Different Means of Anti-Stigma Education}

Joseph Finkelstein, MD, PhD (Assistant Professor); Evgeny Wasserman, MD, PhD (Associate Professor, St. Petersburg State University (Russia)); Oleg Lapshin, MD, MPH (Postdoctoral Fellow) (University of Maryland)

Study Aim: Professionals working with stigmatized populations (HIV-positive people, illicit drug users, psychiatric patients, etc.) often themselves have negative beliefs and attitudes about their clients. There is a need to educate them about stigma and provide anti-stigma interventions. We designed our study to investigate the effectiveness of anti-psychiatric stigma interventions among college students who are trained to provide special education. The subjects were recruited from a major 
urban metropolitan area. Methods: We enrolled 193 college students from the School of Special Education, Herzen Russian State Pedagogic University, St. Petersburg, Russia. Of those, 76 read an article and WHO brochure (reading group, RG) devoted to the problem of psychiatric stigma, and 69 studied an antistigma computer program (program group, PG). The computer program was guided by adult learning theories and provided interactive education tailored to user comprehension rate. Forty-eight students were in a control group (CG) and received no intervention. The second study visit in six months was completed by 153 students. To measure the level of stigma we used Bogardus Scale of Social Distance (BSSD). Results: At the time of their first visit the age of the study participants was $19.1-1.7$ years; of these, $99.0 \%$ were females. After the intervention in the PG the level of stigma decreased from $11.4+/-4.0$ to $4.9+/-4.4$ points $(\mathrm{p}<0.0001)$. In the $\mathrm{RG}$ the level of stigma dropped from $11.6+/-4.2$ to $5.9+/-4.4(\mathrm{p}<0.0001)$. In six months after the intervention the analysis of CAMI scores showed that the level of stigma in the PG was significantly lower than in both the CG and RG, $(11.7+/-4.4$ in CG, $9.6-4.6$ in RG, and in $9.2-4.2$ PG, $p<0.01$ ).

Conclusions. Web-based education or reading anti-stigma materials could be effective in reducing stigma among university students. The effect of computerassisted education was more stable as assessed in six months.

\section{8-06 Ecological Momentary Intervention: Feasibility of Using Personal Digital Assistants to Deliver an Intervention}

Andrea R. Fubrel; Howard Pope; Charsey Cherry; Seth C. Kalichman (University of Connecticut)

Background: Although personal digital assistants (PDAs) are often used to collect data (termed ecological momentary assessment or EMA), to date no research has used this technology to deliver intervention content, which we will call ecological momentary intervention. This method may increase ecological validity, increase convenience and feasibility for participants, decrease measurement error associated with retrospective reporting, and decrease the burden of data entry for researchers.

Methods: Forty-seven people with HIV participated in a brief message-based intervention designed to reduce sexual risk and increase intentions toward using condoms. The intervention was delivered solely via PDAs and consisted of brief messages presented on a PDA three times randomly throughout the day for seven days.

Results: Although sexual risk behavior proved difficult to change, it was feasible and convenient for most participants to use PDAs to deliver interventions. Most participants reacted positively to the intervention and reported enjoying using PDAs. Several participants intended to purchase PDAs to keep track of medication dosing and appointments.

Implications: Ecological momentary intervention may effectively change certain behaviors, particularly high maintenance behaviors such as medication or treatment adherence. Ecological momentary intervention may be used to present booster sessions or supplementary information, and can be interactive and individually tailored.

\section{8-07 How Usable are Current GIS Maps: Communicating Emergency Preparedness to Urban Populations}

Christina Zarcadoolas, PhD; Jennefer Boyer, MPH; Arthi Krishnaswami, MA; Andrea Rothenberg LCSW (Mount Sinai School of Medicine)

Despite the growing popularity of Geographic Information System (GIS) information maps as a tool in public health and emergency preparedness, little known about 
the ability of inner city residents to read and use them. Researchers conducted an intercept usability interview using New York City's Office of Emergency Management Storm Surge Report in East and Central Harlem. Sample consisted of 178 respondents (134 English-speaking, 44 Spanish-speaking). Findings reveal that a majority of less educated adults (those not completing high school) could not read and use the maps for basic and vital information. Based on reading the GIS map $40 \%$ could not point to where they lived, $83 \%$ could not correctly identify what hurricane evacuation zone they lived in, and $83 \%$ did not know how to get to the evacuation center closest to their home. Even among those who had higher education (HS or some/college), 48\% said they did not know how to get to the closest evacuation center. Researchers also found that when it comes to trust in emergency preparedness officials there is reason for concern. Even before residents determined that the map was not clear and decipherable, $40 \%$ said they do not trust emergency directions from city officials.

\section{8-08 Creating A Virtual Community of Practice For Street Nursing}

Dyanne Semogas; Ruta Valaitis; Wendy Muckle; Cathy Crowe; Noori AkbtarDanesh; Anne Childs; In Partnership With Shelter Health Network, Hamilton, Ottawa Inner City Health Inc, McMaster University, MOH-LTC PHIIT (McMaster University)

This paper will report on the progress of an initiative representing the development of a Virtual Community of Practice (VCoP) dedicated to knowledge transfer and exchange for street nursing practice. This web based innovation provides an opportunity for the practice community to: connect and exchange information through on line discussions; learn the most current research through summaries and structured abstracts; offers information regarding daily challenges and solutions; and profiles how street nurses are shaping and changing their clinical practice environments. It represents technology implemented by and for the community of practice.

Methods and Approaches: The project has two phases: 1) the needs assessment and participatory development phase; and 2) the implementation and maintenance phase. Wenger, McDermott and Snyder (2002) stress the importance of fostering a feeling of collective responsibility for communities of practice. The paper will discuss the use of participatory approaches that directly involves stakeholders in order to enhance a sense of ownership and responsibility including: Think Tank Workshops, Think Aloud Usability Testing and Q Methodology approaches.

Wenger, E., McDermott, R., and Snyder, W. (2002). The challenge of distributed communities. In Cultivating Communities of Practice, 1st ed., Cambridge, MA: Harvard Business School. 113-137.

\section{8-09 Web Infrastructure for Treatment Services (WITS): A Publicly Funded Effort to Promote Technology in Substance Abuse and Mental Health Care}

\section{Daksha Arora, Ph.D. (Westat)}

According to an Institute of Medicine (IOM) report, the mental health and substance use $(\mathrm{MH} / \mathrm{SU})$ health care not only lags behind general health care in its use of information technology (IT) but is also not addressed adequately by the current National Health Information Infrastructure (NHII) initiatives. Recognizing the importance of IT in providing quality care and the lack of sufficient use of IT in substance abuse and mental health (SA/MH), the Center for Substance Abuse Treatment (CSAT), a federal agency, initiated a one-of-a-kind publicly-funded 
technical assistance project. The primary goal of this effort was to provide webbased IT infrastructure to various states in the U.S. that would help standardize clinical practices in $\mathrm{SA} / \mathrm{MH}$ and generate standard data that could be aggregated and analyzed for outcomes assessment at the local, state, and national level. This paper describes the development and implementation of the Web Infrastructure for Treatment Services (WITS), a comprehensive, Internet-based electronic health record system for $\mathrm{SA} / \mathrm{MH}$ providers which addresses all clinical and business management functions through its modules of client assessment; treatment planning; discharge; referral; agency, program, and staff management; billing; and a core set of aggregate reports. Two states and one large county piloted WITS in July 2004. By December 2006, 12 states and counties had implemented some form of WITS. Fast electronic sharing of information across different providers resulting in coordinated care; greater involvement of the patients in their treatment and data collection; compliance with state and federal regulations; and standardized data and data collection methods across the state were some of the benefits reported by WITS users at an evaluation meeting in 2006. Old mindsets and habits, political and organizational changes; Internet connectivity issues in remote locations were some of the reported challenges in implementing WITS.

\section{8-10 Influencing Urban Health Policy in India: Through Evidence Generation, Demonstration Programs and Gentle Dialogue for Informing Government on Institution Building.}

\section{Partha Haldar; Rajeev Kumar; S. Kaushik (Urban Health Resource Centre)}

Background: India considered predominantly rural, has traditionally rural focused health and social sector policies. However, rapid urbanization has posed major challenges with increase in urban poverty. The 100 million urban poor of India represent its fastest growing population segment. Health conditions of urban poor are precarious. They receive low policy focus due to their being less powerful political constituency than rural. There are unclear guidelines, inadequate operational structures and weak capacity at all levels. Thus, despite presence of several stakeholders, existing urban health system in most Indian cities is unable to meet growing health needs of urban poor.

Methods: Integrated approach to influence policy at National and State level was adopted. It included technical assistance to government; sensitization and capacity building of state and city officials, study tours to model programs and need based support for developing institutional mechanisms to propel urban health agenda. Key features were: establishing importance of urban health with credible data; beginning through appreciating positive features and opportunities of existing health policies; demonstrating evidence based consultative programming in diverse cities; during this process identifying champions within system, facilitating their initiatives; ensuring involvement of senior officials at critical junctures; facilitating official documentation in Government system; remaining flexible, supportive and responsive.

Outcomes: This approach resulted in increased attention to Urban Health; the National Government has given urban health the priority focus and set up National Urban Health Task Force. There is now clear cut programme focus and dedicated financial allocation for Urban Health at National level. State governments have initiated Urban Health Programs in a phased manner. Nation Family Health Survey (equivalent of DHS) has included urban slums data for eight large cities. 


\section{8-11 Internet Use and Breastfeeding among Low-Income Women}

Joy P. Nanda; Marycatherine Augustyn; Amy Resnik; Susan S. Gross; David M. Paige; Caitlin Cross-Barnet; Angie Wong; Linda Kelly (Johns Hopkins Bloomberg School of Public Health)

The short and long-term health benefits of breastfeeding are well established. Yet, breastfeeding rates in low-income, urban populations remain lower than national rates. The purpose of this investigation was to examine the effects of internet use on breastfeeding among Maryland WIC program participants. We analyzed the relationship between internet use and breastfeeding using databases from 2005-2007. In general, breastfeeding rates were lower than the $75 \%$ national rate. However, exclusive breastfeeding rates among internet users were significantly higher than non-users ( $10.1 \%$ vs. $5.9 \% ; 8.3 \%$ vs. $4.4 \% ; 8.8 \%$ vs. $5.3 \%$ for $2005,2006,2$ qtrs of 2007 , respectively). The odds of exclusive breastfeeding among women who used the internet was almost double that of women who did not use the internet or whose internet usage was unknown. (O.R. 1.8; 95\% CI: 1.6-2.0, p<0.001 for 2005; O.R. 1.9; 95.CI: 1.82.1, $\mathrm{p}<0.001$ for 2006; O.R. 1.7; $95 \%$ CI: $1.6-1.9 ; \mathrm{p}<0.001$ for 2007). Additionally, women participating in WIC in 2005, but not in 2006 or 2007, who used the internet had a $24 \%$ greater breastfeeding rate (O.R. 1.24 ; $95 \%$ CI: $1.15-1.33$, p $<0.001$ ) than women who did not use the internet or whose internet usage was unknown. Significant differences in exclusive breastfeeding rates during each time period of analysis however, persisted between internet users and non-users when geographic residence of the participants, selected maternal and infant characteristics available on the administrative datasets were controlled. While it is not clear how internet usage affects breastfeeding, it is well known that internet usage is increasing and a large percentage of users are seeking out information pertaining to health. Additionally, online internet users are becoming more representative of the U.S. population in terms of race, income and educational attainment. The stage is therefore set for Internet Technology to be harnessed to improve breastfeeding rates in disadvantaged populations.

\section{8-12 Streamlining Data Collection with Technology to Improve Client Services}

Shelley N. Facente, MPH; Dale R. Gluth, MA; Nicolas M. Sheon, PhD (San Francisco Department of Public Health, AIDS Office)

ISSUES: HIV prevention programs are often required to collect data to demonstrate program effectiveness. This impedes the ability of workers to focus on providing risk reduction services. For this reason, UCSF researchers, San Francisco DPH staff, and Magnet, a gay men's community organization, developed a technology package called PalmIT, which collects data from clients in a streamlined, user-friendly, adaptable manner.

DESCRIPTION: PalmIT automates client-level data collection, gathering risk and demographic history from clients prior to their session with an HIV counselor. This improves data quality, allows clients privacy in answering personal questions, and frees staff to focus on risk reduction counseling. PalmIT is adaptable to any setting where client data is collected.

LESSONS LEARNED: Client data can be self-collected in many languages, and can be adapted for clients with low literacy or impaired vision. One person on-site must have some technological ability to prepare devices for use, sync and manage data. Given that, any site can use technology to improve client-level data collection.

RECOMMENDATIONS: The capital investment to purchase hardware and software clearly for PalmIT pays for itself within a short time. The benefits of increased quality of services far outweigh the challenges of changing to a technology-based system. 


\section{8-13 Assessing the Effects of Information Technology in the Delivery of Health Services in Naga City, Philippines}

Maria Leny E. Felix (Center for Reproductive Health Leadership and Development, Inc.)

Introduction: Studies on Naga City Government's use of information technology (IT) in governance have indicated improvements in the efficiency and productivity of personnel and people's access to basic services. However, its impact on health care has not yet been fully assessed. This study examines the effects of the city government's Information and Communications Technology Development Program in the delivery of health services.

Methods: Client exit interview was used to collect data among 30 patients of the Naga City Hospital on their utilization of health services and perception of the quality of care. Focus group discussion with 15 leaders of urban poor associations, interview with local health officials, and review of literature were also conducted.

Results: Overall, the use of IT has improved the management of health care following the computerization of records and procedures. Results of client interview suggested that they were satisfied with the services and up-to-date health information from the government's website. The Emergency Rescue Naga Program which can be accessed through its hotline and VHF Radio was positively assessed. Feedback or complaints concerning health services can also be forwarded through Textserve using a cellular phone.

Conclusions: The initial findings from this study demonstrate the link between IT and improved health care, especially among the urban poor. It is important to encourage local government units to invest their resources in the establishment of IT Program for health care.

\section{8-14 The Online Health Education Curriculum Resource Center}

Leslie Goldman, MA; Janice Kaplan, MLN; Edward Diller, MPH (The New York Academy of Medicine)

The New York Academy of Medicine, Office of School Health Programs and Division of Information Management, in partnership with the National Library of Medicine has developed the Online Health Education Curriculum Resource Center: www.HealthLessons.org. This publicly available Website contains more than 300 free health education lesson plans on physical activity, nutrition, alcohol, tobacco, illicit drugs, injury prevention, and mental health. Each lesson has been vetted by health education experts for accuracy, currency, usability and national health education standards. The Website enables teachers to locate, utilize, and provide feedback on effective health education lessons on the Internet.

Studies have shown that dynamic classroom health instruction can have a positive impact on the health-related behaviors of youth. By providing unfettered access to high quality, up-to-date lesson plans, www.HealthLessons.org provides teachers with resources to educate students on a broad range of health issues. An evaluation conducted of the Online Health Education Curriculum Resource Center in 2006 concluded that teachers across the nation, in Grades K 12, see http://www.HealthLessons.org as a significant resource that enhances their ability to provide classroom health instruction.

Participants at this presentation will have the opportunity to experience an interactive real-time online demonstration of www.HealthLessons.org; examine the innovative design of the Website; review the three year development process, including how lessons were reviewed and selected; and discuss the results of the evaluation conducted in 2006 . 


\section{8-15 Perceptions About Information Technology (IT) among Minority Patients Receiving Care at Community Clinics in Los Angeles (LA)}

Magda Shaheen, MD, PhD; James Smith, MD; Elsa Garcia, MD, MPH; Richard Baker, MD (Charles R Drew University of Medicine \& Science)

Background: Previous studies have documented demographic differences in access/ use of computer/internet. Information is lacking on the perceptions about IT among community clinics' patients.

Objective: To measure the knowledge, comfort, and perceived skills at finding/ evaluating health online resources among patients at community clinics in LA.

Methods: Cross-sectional survey of patients at two community clinics in LA using a questionnaire to interview a systematic sample of 308 patients.

Results: Of 308 patients; $18-82$ years (mean $=45, \mathrm{SD}=13$ ), $78 \%$ were female, $74 \%$ were Hispanics, $44 \%$ were married, $75 \%$ had income $<\$ 25,000,87 \%$ had $<$ high-school education. Using computer/internet at home were $35 \%$ and $25 \%$ respectively. Of the internet users, $31 \%$ looked for health information online. Respondents felt the usefulness of the internet in making health care related decisions $(85 \%)$, and the importance of online access to health resources $(90 \%)$. Participants agreed/strongly agreed about the following 8 -items related to the online resources; knew its availability $(69 \%)$, knew where to find it $(79 \%)$, knew how to find the helpful ones $(79 \%)$, knew how to use the internet to answer health questions $(85 \%)$, knew how to use it to help themselves $(90 \%)$, had skills to evaluate it $(72 \%)$, can tell its quality $(59 \%)$, felt confident in using it to make health decisions $(67 \%)$. The reliability of the 8 -item scale was high (alpha $=0.87)$. Factor analysis produced a single factor $(\%$ of variance $=54 \%)$ that was associated with years of education $\&$ race/ethnicity $(\mathrm{p}<0.05)$.

Conclusions: The level of using the internet to look for health information was low. Most patients who looked for health information believed its usefulness \& importance, had the knowledge, comfort, and perceived skills at finding/evaluating health resources online. An educational intervention to enhance patients' access to online health resources may improve patient's awareness and ability to make health related decisions.

\section{8-16 A Novel, Multimodal Information System for Pre-Visit Alcohol and Drug Screening in General Healthcare Settings Using a Secure Central Data Repository and Exchange.}

C. W. Shanahan; A. Rubin; T. Kim; B. Adams; I. Persand; C. E. Chaisson; M. Brolin; J. Richardson; K. Pressman; D. P. Alford (Boston Medical Center, Boston University Schools of Medicine and Public Health, Brandeis University, Massachusetts Department of (Boston University School of Medicine / Boston Medical Center)

Unhealthy alcohol and drug use are pervasive U.S. public health problems. Although problem behavior identification and brief intervention counseling are effective, routine screening does not occur in general healthcare settings. We report initial development and implementation of a multimodal information system to screen for alcohol and drug use via paper-based surveys and human interviewers, fully automated telephone-based interviews, and web-based applications with intent to screen more than 150,000 patients over 5 years in general healthcare settings within an urban safety-net healthcare system. The MASBIRT (Massachusetts Screening, Brief Intervention, Referral and Treatment) Program uses age-specific, validated screening instruments (ASSIST/CRAFFT) for unhealthy alcohol and drug use. Patients receive an individualized substance use risk assessment based on their 
screening score along with concrete recommendations and follow-up. Data from human-delivered (scanned paper forms and web-based applications) and automated (interactive voice recognition) screening tools are extracted, aggregated, and stored in the electronic medical record for healthcare professionals and a central repository for ongoing project management, patient tracking, reporting, analysis and data exchange. Required substance specific data is reported electronically to federal and state agencies. We demonstrate that development and implementation of a flexible automated data collection system using a secure central data repository for screening and reporting in general health care settings is possible.

\section{8-17 Barriers to Bridging the Digital Divide to Promote Urban Health}

Sara Minsky; Vish Viswanath; Emily Kontos (Dana-Farber Cancer Institute)

Almost 70 percent of US adults, about 150 million people, are estimated to use the Internet. Of these, approximately 80 percent have gone online to look for health information on topics including diet, fitness, drugs, hospitals, new treatments, alternative medicines and doctors. Millions of websites covering these health topics have been developed in response to this interest. Despite the widespread diffusion of the Internet, people with a lower socioeconomic position -education and income, and certain racial ethnic groups, have lower access to the Internet. Such divisions are likely to have profound consequences for public health. As part of formative research informing an intervention aimed at developing peoples' capacity to seek and use heath information on the Internet, we conducted two focus groups with 16 adult literacy students in the Boston area to learn more about their Internet experiences, and barriers. While most individuals reported using the Internet at their school or library, few reported home Internet access. Of those who used the Internet, the majority reported challenges with finding information including getting lost easily, and difficulty researching a topic. These findings suggest that the underserved have limited home Internet access and experience challenges navigating the plethora of health related websites.

\section{8-18 Evaluation of Information Quality of Childhood Infectious Disease on the Internet} Clement Azodo; Michael Omili; Martins Ozor (HOSPITAL)

Background: Internet offers easily accessible health information for the populace.

Objective: To determine the information quality on childhood infectious disease in Internet

Methods: The search term "mumps" was entered into five commonly used search engines (MSN, Yahoo, Google, HotBot and Metacrawler). The first 40 links displayed by each engine were evaluated for a theoretical total of 200 websites. Information quality score of $0-12$ was generated for website evaluation. A score $0-4$ represented low quality, 5-8 moderate quality and 9-12 high quality.

Result: 28 out of 200 websites that met inclusion criteria was evaluated Relevance ratio of search engine calculated was 0.14 . 10(36\%) were of high quality, $18(64 \%)$ moderate quality and none was of low quality. Mean quality of information was $66 \%$. Those website that displayed HON code are $5(18 \%)$ and $3(60 \%)$ were of high quality rank. Majority 23(82\%) mentioned the value of vaccine in prevention. More than half $16(57 \%)$ displayed ownership of website. $17(61 \%)$ have interactivity opportunity, 7 $(25 \%)$ displaced author and $9(32 \%)$ disclaimer notice. Only $1(4 \%)$ indicate herbal treatment $.18(64 \%)$ gave out relevant date of update and review.

Conclusion: Information quality of childhood infectious disease in internet are predominantly moderate quality and thus a relevant source of health education. 


\section{8-19 Reducing ER Visits through Video Technology}

\section{Heather Barton; Elizabeth Borycki (vancouver coastal health - Community)}

Health Care in the 21st Century has become more complex and LOS in hospital has doubled. In an effort to disperse the demands on acute care, the health care division of community home care has expanded exponentially. Patients are readily being managed in their home with more acute interventions. This has been fabulous progress that supports health care needs.

However, we are seeing an incredible rise in emergency room visits. In some areas the wait time is 4-6 hours, and patients are being housed in the hospital corridors while waiting for a hospital bed to become available.

The power in Information Technology has not been accessed. For instance there is an ability to support patients in their home, support home assessments, reduce the demand on direct physician intervention and reduce the number of patients seeking service at the emergency room with video imagery. Examples of technology support for reducing ER visits include home video assessments (psychiatry) and wound management (Pixilaire).

Our presentation will review an untapped network of support to help reduce the strains on the current practices in health care system.

Thank you

\section{8-20 Computer Literacy, Health Technology and Behavior Change}

Edward Scott, MD; Everett Logue, PhD; Dena Bartko, BA; Lori Milo, PhD; William Smucker, MD (SUMMA Health System)

Objective: Assess computer literacy among urban primary care center adults to support planning for asynchronous patient-provider communication via an Internet patient portal, email, computer kiosks, or enhanced telephone/voicemail systems. Proactive communication between multidisciplinary care team members and patients is an essential aspect of the Chronic Care Model.

Design: Waiting-room survey $(\mathrm{N}=425)$.

Results: Internet/email use decreased strongly in the two older age groups $(70 \% \mathrm{v}$ $44 \%$ v $26 \% ; \mathrm{P}<.0001)$. Young disadvantaged patients reported a higher usage rate than older advantaged patients $(61 \%$ v $42 \% ; \mathrm{P}=.0004)$. [In a logistic regression model of Internet usage, with age group and an economic class indicator as predictors, the age group estimate was significant $(\mathrm{P}<.0001)$, while the class indicator was not $(\mathrm{P}=.52)$.] Greater than $50 \%$ of users expressed desire for increased communication with their provider via internet; less than $25 \%$ of users supported charging fee for this service.

Conclusions: Study data suggest that at least $50 \%$ of patients sampled have sufficient computer skills to interact with their providers via an internet patient portal or similar technology. However, a telephone/voice mail system may be useful to support the chronic care model for those older patients with the lowest computer literacy levels.

\section{COMMUNITY APPROACHES TO URBAN HEALTH}

\section{9-01 Responding to Community-Identified Health Concerns: Hip-Hop as a Way to Resolve Conflict}

Saurabh Saluja, BA; Kirsten Austad, BS; Sean Spencer, BS; Sharon Younkin, PhD (Allied United for Health)

University students, in collaboration with non-profit organizations, implemented a conflict resolution program in an urban underserved neighborhood. Prior neighborhood assessments indicated conflict as a community-identified health concern. 
Local artists and an educational instructor were recruited to teach youth about hiphop as a healthy means of communicating and resolving conflicts. Participating youth learned about the roots of hip hop, as well as how hip hop can be used to negatively influence people. Instruction included strategies to transform personal adversity into improved self concept and techniques illustrating how to use artistic expression to create social change.

The program culminated in an event in which local positive-themed hip-hop artists and program participants performed for the community. University students also interacted with area youth, further developing a connection between the neighborhood and the university. Youth were encouraged to express themselves positively, use their talents to strengthen their community, and regard student volunteers as positive role models.

A limitation was the low number of youth that developed their own artistic material. Future projects will begin youth recruitment earlier and use outdoor events to improve participation. Still, our preliminary feedback indicates that collaborations between the underserved neighborhood and the academic institution were viewed favorably by the community. Such collaborations must pursue creative means of promoting health and wellness that address community-identified concerns.

\section{9-02 Informed Neighbourhood Groups have Positive Influence on Maternal and Child Health Practices in the Households}

Karishma Srivastava; S. Kaushik; Rajeev Kumar (Urban Health Resource Centre)

Introduction: The sub optimal health seeking behaviour at households in slums with respect to hygiene, pregnancy care, delivery practices and child care are found to have a cumulative adverse impact on lives of mothers and children.

Neighbourhood practices influence health seeking behaviour among urban poor households. These behaviours are reinforced through informal social processes in the neighbourhood.

The methodology: UHRC devised a strategy to influence household health behaviours through informed neighbourhood groups. The groups promote healthy maternal and child health behaviours using simple communication technologies: instruments like flip charts, pictorial posters, job aids during monthly mothers meetings. The informal meetings during day to day social transactions also serve as contact points for information sharing.

These groups usually start as a nucleus of early adopters. Early adopters of healthy behaviours play crucial role in influencing others in the neighbourhood by showcasing the benefits of preventive healthy behaviours and reinforcing them via household visits during critical junctures of pregnancy and child birth. Activities like Anaprasanna (ritual of initiating cereals to infants), healthy baby and mother competitions reinforce this in the consciousness of people. The community health workers provide information to group members and help them with communication tools.

Outcome: Groups have had a positive influence on adoption of healthy behaviours in Indore and Agra Urban Health programmes, covering around 3.5 Lakhs slum population. A post intervention evaluation in Indore shows significant positive change in household behaviour. Percentage of mothers discarded colostrum decreased from $66 \%$ to $51 \%$. Exclusive breast feeding upto 3 months increased form $23 \%$ to $46 \%$ in programme slums. 


\section{9-03 Medical Students and a Community Wellness Center Collaborate to Address Urban Health Issues}

S. L. Younkin; S. Corrado; T. W. Franklin-Ford; O. A. Adeyemi; O. A. Ehie (University of Wisconsin School of Medicine and Public Health)

Students at the University of Wisconsin School of Medicine and Public Health were awarded an American Association of Medical Colleges (AAMC) "Caring for Communities" grant to support a formal programming partnership with the Allied Wellness Center in June 2006. The Allied Drive neighborhood is a small urban community in Madison, WI. The neighborhood is home to the highest concentration of needy families in the county, with $95 \%$ of children participating in school free and reduced lunch programs and $41 \%$ of residents earning less than $\$ 15,000$ annually. The community is plagued by crime, violence, substance abuse, and hopelessness. The Allied Wellness Center is a small (one half-time employee) nonprofit agency located in the heart of this neighborhood. The Center was established in 2004 in response to requests from area residents, and is designed to promote health and wellness of body, mind and spirit. The Center provides basic health care and advocacy, serving as a place of respect, empowerment and pride for the community. Working in partnership with the Center's Community Parish Nurse, students surveyed area residents about their health concerns and barriers to health and well being. Next, students worked with the Center's community parish nurse to design and implement health related programs targeting the issues identified by the residents. Medical students addressed: physical fitness, literacy, self esteem, violence, HIV/AIDS and asthma. While it is too early to evaluate the broad impact of these programs, neighborhood residents attending programs have rated them highly and indicated that they gained valuable knowledge and information. It is hoped that, by the end of the AAMC funding in 2010, this over-burdened urban neighborhood will be transformed into a powerful, positive, healthy community.

\section{9-04 Preventive Health Behaviors in Low-Income Urban Caucasians}

J. Bowie, B. Curbow, L. Dubay, H. Juon, T. Laveist, L. Lebrun, R. Thorpe (johns hopkins university bloomberg school of public health)

Background: Urban Caucasian populations in the United States have not been widely considered in studies addressing health disparities and their poorer segments have largely gone unrecognized in public health research.

Methods: Data from the Southwest Baltimore Study (SWB) included lowincome Caucasian and African Americans with similar demographic patterns. Data for low-income populations from the National Health Interview Survey (NHIS) were also examined.

Results: Preventive health behaviors for Caucasian SWB respondents were below the expected levels for the nation. Compared to prevalence estimates, the percent of current cigarette smoking was $58.8 \%$ (23.1\% nationally); for women mammography use in the past two years was 50\% (70\% nationally) and Pap smears received in the past two years was $61.5 \%$ (90\% nationally); and only $40.7 \%$ of individuals 50 years and older had been screened for colon cancer in the past two years (50\% nationally). An additional indicator of less than optimal health included $37.4 \%$ reporting their health as fair to poor. Data from the NHIS showed consistent results with mammography use at $56 \%$, pap smears at $74 \%$, and colon cancer screening at $18 \%$. 
Conclusion: This study sheds light on the need for greater awareness of health disparities in Caucasian urban populations who may share health risks with their poor urban counterparts. Research attention as well as intervention is needed to improve the practice of preventive health behaviors, health access, and outcomes for urban populations including those that may typically fall under the radar screen for health disparities.

\section{9-05 Findings}

Robyn R.M. Gershon, MHS, DrPH; Monika Pogorzelska, BA; Patricia W. Stone, RN, PhD; Kristine A. Qureshi, RN, DNSc (Mailman School of Public Health, Columbia University)

Introduction: Home healthcare workers are key responders during a pandemic. In NYC, their role during an avian influenza outbreak will be to provide home care to sick or quarantined individuals at home. Yet information regarding their willingness to respond during these types of events is scarce.

Methods: Items related to willingness to provide care were included in a 5-page anonymous questionnaire designed to assess the occupational health hazards associated with home care. The questionnaire was administered to over 3000 home healthcare employees. Both qualitative and quantitative data collection methodologies were utilized.

Results: Only $12 \%$ of home health aides $(\mathrm{N}=1600)$ said that they were willing to provide care to patients on home quarantine during an outbreak. Registered nurses were willing to provide home nursing care during outbreaks of: anthrax $(35 \%)$, pandemic influenza (37\%), SARS (38\%) and smallpox $(42 \%) .48 \%$ stated that they would provide care to patients in home quarantine.

In focus group sessions, concerns about reporting appeared to be related to fear of contagion and lack of personal protective equipment.

Conclusion: Training, education and the provision of safe and effective personal respiratory protection is probably the most effective approach to supporting the responsiveness of home healthcare workers during a pandemic.

\section{9-06 US Tobacco Control Not Yet Realized in Young, Urban African-American Adults Age 18-24 Years}

Frances Stillman, EdD (Bloomberg School of Public Health, Johns Hopkins); Lee Bone, MPH, RN (Department of Health, Behavior and Society, Johns Hopkins University, Bloomberg School of Public Health); Erika Avila-Tang, PhD, MHS (Institute for Global Tobacco (Johns Hopkins University Bloomberg School of Public Health)

Cigarette smoking is the leading preventable cause of both morbidity and mortality in the United States. An at risk population of great concern are the 18-24 year olds who are being targeted by the Tobacco Industry. Studies have looked at young adults attending college but little if any information exists on the high-risk population of unemployed, underemployed, or blue-collar, inner city African American young adults. Little is known about the social, cultural, and environmental factors that might be influencing the high smoking rates is this inner-city population. Using the principles of community-based participatory research, a survey of over 300 urban African American adults 18-24 year olds enrolled in employment training and education programs in Baltimore City was conducted. Smoking behaviors, tobacco acquisition strategies and smoking norms were assessed. Findings indicate that a) smoking remains an overarching normative behavior with prevalence of tobacco use currently over $50 \%$; b) an important source of cigarettes for these young adults are single cigarettes (loosies), which are widely 
available throughout the community for purchase, over $75 \%$ of young adults report purchasing loose cigarettes over the last month. This presentation will discuss these survey results and discuss potential strategies to address this significant urban public health problem.

\section{9-07 Community Based HIV Testing: Experience from the Nairobi Urban Demographic Surveillance System, Nairobi, Kenya.}

John Kebaso; Samoel Khamadi; Abdhalah Ziraba; Chi-Chi Undie; Matilu Mwau; Nyovani Madise (African Population and Health Research Center)

Objectives: To describe the methods used in the Demographic Surveillance system to collect data on the prevalence of Immunodeficiency virus (HIV) and address the value of DSS data to country HIV surveillance systems.

Methods: During the year 2006-2007, dried blood spot samples were collected for HIV testing, following internationally accepted ethical standards in an urban informal settlement of Nairobi. We used the Nairobi Urban Health Demographic Surveillance System (NUHDSS) membership (from the data set) as our sampling frame. A list of eligible participants was generated using the eligibility criteria of age and sex (i.e. males of 15-54 years old and females of 15-49 years old). Equal numbers of either sex were randomly selected using computer generated random numbers till the required sample for each site was obtained. The results were presented by age, sex, ethnicity and social economic differentials. To estimate the effects of non-response, HIV prevalence among non-responding respondents was predicted using multivariate statistical models for tested, with a common set of predictor value. Analyses were performed using STATA/SPPS. The results of HIV and NUHDSS-surveillance were anonymously linked with demographic and behavioral data collected from individuals through the NUHDSS. Descriptive analysis was done on demographic characteristics and potential behavioral risk factors. Following this, risk factors for prevalent HIV infections were explored in a multiple logistic regression model.

Results: The preliminary results from the pilot survey indicate a sero prevalence of HIV in Korogocho slums at $13 \%$. Rates of HIV testing varied from $15.1 \%$ among women and $12.7 \%$ among men in Korogocho slums. These rates are relatively higher than those of the general Nairobi population which are estimated at $10 \%$ (KDHS, 2003). Consistent patterns of HIV infection were observed by age and sex, with considerably higher rates among women especially at younger ages.

\section{9-08 Identifying Unlisted and Hidden Slums and assessing Health Vulnerability of Slums}

Siddharth Agarwal; Karishma Srivastava; S. Kaushik; Partha Haldar; Rajeev Kumar (Urban Health Resource Centre)

Background: Programs developed and implemented by agencies external to slum are often not able to reach the neediest. They usually reach to small proportion of urban poor, often the relatively better off residing in officially listed slums. Conditions in certain slums are found worse in comparison to those in others. There is need to reach all slums for better impact.

Methods: This methodology first piloted in Indore involved identification of all slums starting with lists available with various urban agencies. More were identified through community consultation and were mapped. Qualitative criteria were developed for assessing vulnerability of slums and urban poor clusters through consultation with communities. A mechanism for categorizing slums on a grade of 
differential vulnerability emerged. The whole process was accomplished utilizing local knowledge of slum communities, grass root volunteers and workers.

Results: Key factors which intensify health vulnerability include: economic conditions; social conditions- education, traditional pregnancy and birth care practices, alcoholism; living environment- security of tenure, roads, drainage; access and usage of public health services; disease incidence; collective organized community effort. Of a total of 539 slum locations, 115 were unlisted or hidden slum clusters and 156 were assessed as vulnerable.

Implications: Identification, mapping and assessment of all slums is important for locating the hitherto missed out slums and also for focusing on the neediest slums. The slum assessment technique has emerged as a valuable tool for targeting efforts and resources. Acknowledgement and inclusion of local vulnerability factors help in better understanding of the specific needs, developing context appropriate intervention strategies. The process being participatory also helps in engaging slum community and grassroot workers in programme planning.

\section{9-09 Healthy Choices for Baltimore: Addressing the Need for Nutrition Education in Urban Environments}

Poornima Vanguri (University of Maryland, School of Medicine)

"Healthy Choices for Baltimore" is a nutrition education program created to promote healthy eating and exercise habits among children and adolescents in Baltimore city. It also aims to reduce childhood obesity and its co-morbidities, a rising epidemic in the United States. The program was initiated in 2006 by a University of Maryland medical student when it was recognized that such programs do not exist in the health curriculums of many city schools. The program is sustained through a collaborative effort between the medical school and its administration, local businesses, as well as teachers and parents of students attending Midtown Academy, a public charter school in Baltimore City. Volunteer medical students from the University of Maryland teach nutrition classes to grades 3 through 8 . The medical students have been given a community service opportunity to address a growing health issue and to help children who live in the city. The volunteers get a chance to use their classroom knowledge in a practical and enjoyable manner while personally interacting with community members. The lesson plans address obtaining optimal nutrition with limited resources, as well as the importance of exercise and physical activity. They also include hands-on activities, demonstrations and taste-testing. The program has been highly successful and strongly received. Midtown Academy students have made strides in understanding how to make healthy food choices and have learned the importance of preventing future health problems through lifestyle changes. This program creates a knowledge base for health behavior change of children who would not otherwise receive this necessary information.

\section{9-10 What's the Problem and who has it? An Application of Innovative CBPR}

Yanique Redwood, MPH; Marshall Kreuter, PHD; Margaret Hooker, BS; Catherine Prather (Institute of Public Health - Georgia State University)

What's the problem? Who has it? Why them? These are the key questions that epidemiologists seek to answer when faced with the task of establishing health priorities. The hallmark of community-based participatory research (CBPR) lies in the respectful covenant that emerges when community residents join together with 
interested organizations and institutions including health researchers, to share fully in all aspects of decision-making including the identifying priority health problems and the complex determinants that influence those problems.

Accountable Communities: Healthy Together employs CBPR methods to promote a sustained effort to redress the social, economic, and environmental determinants of health disparities experienced by the 16,700 residents of Neighborhood Planning Unit V (NPU V), a cluster of five inner city Atlanta neighborhoods. This paper describes how information generated by quantitative and qualitative methods (perception analyzers, GIS mapping, extant data, and Photovoice) helped clarify NPU V residents' perceptions of the health, social and economic priorities in their community. The priorities that emerged and shaped subsequent intervention pilots surprised many.

\section{9-11 Building Capacity to Address Social Determinants in an Urban Setting: A Community Partnership Approach}

Dr. Davison Munodawafa; Dr. Cherian Varghese; Dr. Jostacio Lapitan; Dr. Tandav Murthy (WHO South-East Asia Regional Office)

This presentation highlights the development, application and utility of the BHUP (India) jointly, coordinated and managed by WHO SEARO, WHO India Country Office and WHO Kobe Center, Japan in collaboration with Bangalore Municipality to address socially determined health problems affecting urban population. A functional partnership model to address identifiable social determinants of health in Bangalore City is discussed with a focus on role delineation between WHO, the local municipality and the community including NGOs. First, the presentation focuses on the developmental phase of the proposed intervention which initially trained three local health professional leaders to advocate for the promotion of health Prolead. The second phase established 7 Healthy Urbanization Learning Circles (HULC) to study the specific needs and assets of each given locality that had been identified as an implementation site. The learning process was guided by operational research. Third phase engages communities to intervene through participatory approaches following the development of work plans and training modules. The main health problems identified and addressed include noncommunicable diseases, water, sanitation and housing, recreational amenities, violence prevention, safety promotion and malnutrition among children. The efficacy of the partnership model is presented using evidence from the on going monitoring and evaluation.

\section{9-12 University of Maryland's Model for Community Engagement and Research Partnerships}

S. I. Mishra (University of Maryland School of Medicine); C.R. Baquet (of Maryland School of Medicine); J. Bramble J(Times Community Services); B. Blakely (University of Maryland Eastern Shore); T. Lawlah (The Maryland Center at Bowie State University); (University of Maryland School of Medecine)

While the nation's overall health status has improved over the past decade, disparities exist between whites and racial/ethnic minorities, individuals who reside in medically underserved rural communities and low income whites. These disparities have been compounded by ineffective community engagement and community-based outreach and information dissemination.

The University of Maryland's Comprehensive Center for Health Disparities Research, Training, and Outreach (the UMB EXPORT Center), through its 
Community Outreach and Information Dissemination (COID) Core, has implemented a model community engagement program. The focus of this program is to:

1. foster mutually beneficial community-based partnerships based on trust and respect, and enhance community capacity and empowerment

2. develop and implement collaborative community education programs such as the Mini-Med School programs

3. develop and implement collaborative research projects

The COID Core has integrated the UMB EXPORT Center into Maryland communities as a disease prevention and health and science education resource. In addition, the Core provides leadership and serves as a catalyst for community capacity enhancement, development and dissemination of culturally appropriate disease prevention and health promotion information based on community needs. Equally importantly, the Core promotes and expands the ability of collaborating institutions and organizations to foster, coordinate and conduct evidence based, culturally appropriate community outreach and dissemination, and research.

\section{9-13 Improving HIV Prevention Programs through Technology and Collaboration}

Dale R. Gluth, MA; Shelley N. Facente, MPH; Nicolas M. Sheon, PhD (Magnet)

ISSUES: Community providers, researchers, and policymakers all work to improve services to the population. Each entity brings strengths and weaknesses as well as differing interests and perspectives to any collaboration. HIV prevention programs are frequently resource poor and burdened by administrative responsibilities. This makes them a prime subject for such collaboration.

DESCRIPTION: UCSF researchers, San Francisco DPH policymakers and Magnet, a gay men's community organization, came together to create an intervention to reduce paperwork for HIV test counselors and enhance client services. The collaboration resulted in a multi faceted evaluation of the intervention's effect on counseling, data, client satisfaction, and counselor training.

LESSONS LEARNED: Magnet provided logistics in a community setting, and gained the intervention itself. UCSF provided evaluation and funding, and gained insight about the target population. The SFDPH provided institutional support as well as potential for wider application, and gained understanding of community needs. The result was an intervention that improved client and counselor satisfaction, and has a strong foundation for broader application.

RECOMMENDATIONS: It is vital that HIV prevention entities work together to improve the health of urban people. By combining skills and resources, we maximize our strengths and have the greatest impact on service quality and efficiency.

\section{9-14 The Afya Project: Creating Change Agents for Health and Healing}

I. Shevon Harvey; Imani Bazzell; Lenita Epinger; Wamiatha Sullivan; Karen C. Simms; Carla Hunter (University of Illinois)

This civic commitment initiative, the Afya Project, seeks to nurture healthy lifestyles and community activism among African American women. The Afya Project is collaboration between community and academic partnership in Champaign Illinois, which is considered an urbanized area. Using a community health worker intervention strategy that is informed by the lay health advisor model initially we recruited 45 local African American women to become health educator activists and 
15 students from a local university in Illinois to develop a health empowerment curriculum and training program. In this presentation, we will describe and analyze the strategies used by the health educators activists to increase awareness for hypertension and diabetes among members of their social networks. We will discuss the application of a social network theoretical framework in the development and evaluation of this intervention. We will present results from quantitative and qualitative data gathered from the health educator activists to evaluate the implementation and effects of this social network intervention. Finally, we will discuss lessons learned regarding the use of a community based participatory research process in all aspects of the development, implementation, and evaluation of this theoretically grounded lay health advisor program.

\section{9-15 Community Based Intervention System}

Mike Bailey; Serena Parker (Johns Hopkins University/Center for Communication Programs)

A Baltimore City Council sponsored report released nearly four years ago makes clear what needs to be done to address what the report referred to as a health crisis. Much of the problems outlined in the report concern insufficient coordination and lack of integration of services by providers. Recommendations from the report include a need for a centralized repository for information about prevention funding, activities, and providers and more sharing and dissemination of results by research institutions with organizations involved in HIV prevention. In Baltimore, unrealized social capital resides within the community and their representative community based organizations or CBOs. The Community Based Intervention System (CBIS) seeks to exploit the Internet to develop social capital directed towards the creation and maintenance of an active network of health agents consisting of CBOs, local and federal health agencies and research institutions. Better resource allocation, improved service coordination and more effective health interventions will result from providing an effective means for collective action. Using vector based mapping and rich client technologies, the goal is to develop a fully functional, web based resource that creates a network of organized reciprocity for better health. In a functional sense, the CBIS provides guidance to basic questions concerning health interventions including:

Who is conducting the interventions? Addressed through the integration of a Resource Directory. How are they going to conduct each intervention? Addressed by providing access to relevant data using the Connect to Protect decision making framework. Where are they conducting their interventions? Addressed through vector based mapping allowing $\mathrm{CBOs}$ to select the census block groups where interventions occur. What are they trying to achieve? Addressed through a process that allows users to link program goals to measures available within the system.

\section{9-16 CHOICES (Changing Health Outcomes by Improving Cardiovascular Education and Screening): Piloting a Faith Based Community Intervention Program to Address Disparities in Cardiovascular Disease}

Rosalyn P. Scott, MD, MSHA; B. Waine Kong, PhD, JD (Association of Black Cardiologists)

Background: Although cardiovascular disease (CVD) mortality has decreased, the decline has been less among African Americans resulting in increasing disparities. The Association of Black Cardiologists has focused on faith based interventions such as CHOICES (Changing Health Outcomes by Improving Cardiovascular Education and Screening) to advance self sustaining health promotion capacity in communities. 
Methods: Conduct pilot program comprised of training Health Promotion Specialists, cardiovascular disease risk factor screenings, Bible study curriculum 7 Steps to a Spiritually and Physically Healthy Heart, and Health Ministry Empowerment Conferences.

Results: Twenty churches in Philadelphia, Detroit, Chicago, Phoenix and Clairborne County, Mississippi participated. One hundred and sixty seven congregants were trained as Health Promotion Specialists and certified to be blood pressure, glucose, cholesterol and Body Mass Index (BMI) screeners; and, 4,805 community individuals were screened. Sustained improvement in health related behaviors and knowledge was evident at 12 weeks among the 193 completing the 7 Steps curriculum.

Conclusions: Faith based cardiovascular disease intervention programs can be successfully administered by lay Health Promotion Specialists. Provided with tools and appropriate technology, churches can identify and effect reduction in cardiovascular disease risk factors; and, support and sustain positive health behaviors. We believe CHOICES is rich with key strategies that can narrow the disparity gap and drive future opportunities for community activism in health promotion.

\section{9-17 Visual VoicesTM: An Innovative Tool for Engaging in Community Based Partcipatory Resaerch}

Michael A. Yonas, DrPH; Jessica G. Burke, PhD (University of North Carolina at Chapel Hill)

This presentation will introduce the Visual VoicesTM project and discuss its use within the field of Community Based Participatory Research (CBPR). Visual VoicesTM is an arts based initiative designed to bring youth and communities together within a common artistic venue to address issues concerning their lives, communities, and futures. Youth, as co facilitators, are invited to reflect collectively on a topic, such youth violence, dating violence, and community safety through creative sessions using the mediums of painting, drawing and writing. The multi layer exhibition includes children's interwoven painting placed underneath a transparent visual layer depicting their writings/drawings copied onto acetate. Photographs of the youth taken during the sessions are integrated into the final exhibition. The process and exhibition serve as dissemination platform which all involved learn more about the topic, themselves, their communities. To date, the Visual VoicesTM project has exhibited in eight cities and is over 1800 feet in length involving the creative efforts of over 900 youth from all over the country. Examples from past projects will be shared and specific attention will be paid to discussions addressing the use of Visual VoicesTM as an innovative tool for Community Based Participatory Research.

\section{9-18 Women Empowerment and Health: The SEWA Case Study}

Dr. Davison Munodawafa; Ms. Bijalan Bhatt; Ms. Mirai Chatterjee; Prof. Surinder Aggrawal (WHO South East Asia Regional Office)

The presentation is based on findings from a Case study supported by WHO SEARO in collaboration with SEWA (Self Employed Women Association). Data sources are stakeholders including key informants, policy makers and beneficiaries of SEWA. In addition, a review of documents including annual reports, evaluation reports, and publicity or promotional reports in order to document growth and development of SEWA in terms of its management, coordination and functions was done. Evidence of the effectiveness of strategies used by SEWA to address upstream social determinants of health inequities among women is presented. Finally, an analysis of all information gained from interviews and review of the literature was conducted in order to capture the deep viewpoint of SEWA actions and contributions. 
The overall goal is to present general and specific contribution of SEWA in the empowerment of both urban and rural poor women to tackle social and economic determinants of health through innovative and practical approaches to accessing health care services and housing. Key features of SEWA that are relevant to its performance in an integrated and inter sectoral manner to address health outcomes among poor women are identified.

\section{IMMIGRANT \& REFUGEE POPULATIONS}

\section{0-01 Health Status and Related Complexities among Refugees of Urban Disasters}

Audil Rashid; M.N. Adnan; I.Z. Qureshi; Faiza Naseem (F.G. Degree College (Men))

Undernutrition and health anomalies are linked to each other. In a refugee population the former is a challenge while latter is the ultimate cost or end result. Migratory earthquake victims and Afghan war refugees were investigated for their health status and food requirements. Qualitative assessment surveys and interviews were conducted. Several poor health groups were identified among earthquake victims that include orphans, children separated from their families, disabled due to injury and adolescent females. Migrants from urban earthquake hit areas were more psychologically disturbed due to job/earning loss while youth were in dismay due to virtual damage of all educational institutions. Families without male earning members were most affected by malnutrition. Because of gender inequality among refugee families girl child were not given preference for food access/intake over boys. Children between ages of 6-12 years felt insecure and appear susceptible to be exploited emotionally and sexually. Low HIV prevalence $(0.01 \%)$ was recorded overall but high degree of undernutrition emerged as imminent issue. We conclude that tripartite approach must be followed to bring behavior change regarding gender sensitivity issues by increasing access to food, shelter, education and employment. Technology based educational and health facilities would overcome the existing insecurity among refugee populations.

\section{0-02 Conceptualization and Measurement of Socioeconomic Status of Immigrants to Canada}

Farah N. Mawani, MSc, PhD Candidate (University of Toronto)

Statement of Purpose: It is well established that socioeconomic status (SES) is an important determinant of health. It is particularly important to examine the influence of SES on the health of immigrant populations in Canada because: Canada accepts proportionately more immigrants than any other country; immigrants experience the well-documented healthy immigrant effect; and there has been a dramatic decline in the economic status of immigrants in Canada over the past twenty years, with a growing incongruence between their education and occupation levels.

Methods: This paper will provide an overview of the socioeconomic profile of immigrants to Canada, examine the utility of standard SES measures for immigrants, and propose underemployment as an alternate SES measure that incorporates the potential negative correlation between immigrant education and occupation levels. The paper will then use data from Statistics Canada Canadian Community Health Survey Mental Health and Well-being (CCHS 1.2) to compare the association of SES to 12 month period prevalence of depression (major depressive episodes) using underemployment (a constructed variable) compared to standard measures (highest 
level of education, current occupation, household income adequacy) of SES. The sample will be restricted to labour force participants aged 18-64.

Results: Logistic regression analyses will be presented that estimate odds ratios associating SES with major depressive episodes in the 12 months prior to the survey. First, analyses using underemployment as the primary independent variable will be presented. Second, analyses using income, education and occupation as primary independent variables will be presented.

Conclusions: The results of these analyses will be compared to assess whether the association of SES to depression is measure-dependent; i.e. whether underemployment, is an alternate SES measure that is particularly relevant for immigrants.

\section{0-03 Socioeconomic Disparities in Low Birth Weight among Recent Immigrants to Urban Ontario. The Contribution of the Neighborhood of Residence and the Country of Origin.}

Marcelo L. Urquia; John W. Frank; Richard H. Glazier (Centre for Research in Inner City Health)

The association between material deprivation and adverse birth outcomes is well documented. Little is known, however, about socioeconomic disparities among recent immigrants to urban areas.

To examine this question we linked Ontario's hospital records with the Landed Immigrant Database and assembled a cohort of all women immigrating to Ontario's census metropolitan areas in 1993-1995 who had a first Canadian infant liveborn within 5 years after arrival $(\mathrm{N}=26,989)$. Factor analysis was performed to obtain a neighborhood material deprivation score, which was collapsed into quintiles. The World Bank Classification of Country Economies was used to classify mothers' countries of origin into five income levels. We used logistic regression to estimate the effect of material deprivation in a series of models with increasing degree of adjustment for covariates.

The neighborhood deprivation gradient in low birth weight is attenuated after controlling for maternal characteristics, especially for the income level of the country of origin, which remained highly significant in the full model (OR, 95\%CI low versus high income countries: $2.13,1.51-2.99$ ).

Our findings suggest that self-selection of immigrants into neighborhoods contributes to the income gradient in low birth weight. Strong disparities according to the country of origin prevail among recent immigrants to urban Ontario.

\section{0-04 Explaining Health Outcomes among Immigrant Populations: Assimilation,} Behavioral or Structural?

\section{P. Rafael Hernandez-Arias (DePaul University)}

Objective: Health investigators have demonstrated that although recent immigrants enjoy better or more favorable health than native-born, this positive health status declines within ten years of residence in the United States. The objective of this project is to understand how health investigators explain health outcomes among immigrant populations.

Method: This is a systematic review of articles published from 2000-2006 on immigration and health. A Medline search on emigration, immigration, humans, and United States yielded 769 articles. We selected 124 articles that were empirically-based and in which immigration was the primary or independent variable. Excluded were articles related to U.S. policies, health professionals, welfare, race and ethnicity, and disease screening practices. We conducted a text analysis of the articles using a structured coding instrument. 
Findings: Knowledge on the health status of immigrant populations is primarily based on aggregate data and on comparisons between natives and non-natives or population categories. Investigators explain health outcomes among immigrants primarily as functions of culture, acculturation, behaviors, assimilation, and socioeconomic status. More than often investigators refer to these factors as working in combination. Socio economic status is often limited to income and seldom exploded. Factors such as work conditions, life routines, living environments, and access to foods are seldom mentioned.

Conclusion: The focus on individual culture, behaviors and socioeconomic status as explanations is delimited by underlying ideological and theoretical commitments. Immigrant status engenders unequal social relations that actively generate a multipoints social disadvantage. Identifying how the multi-points social disadvantages are manifested in social interactions will facilitate understanding the individual and social factors that contribute to the rapid decline of the health status of immigrant populations.

\section{0-05 Speaking Together: Improving Care for Patients with Limited English Proficiency}

Marsha Regenstein, PhD (Associate Research Professor), Melissa Stegun, Research Scientist (Department of Health Policy, The George Washington University Medical Center)

Patients with limited English proficiency (LEP) are more likely to experience a medical error or adverse event and a lower quality of health services (IOM, Unequal Treatment: Confronting Racial and Ethnic Disparities in Health Care, 2002). Language barriers also impair discussions of symptoms and treatment regimens, resulting in misdiagnoses, potentially fatal medication errors and poor patient outcomes.

Speaking Together is a national program aimed at identifying, testing and improving the ways that hospitals provide language services to LEP patients. Through a learning collaborative of 10 hospitals, strategies for improving patient safety are assessed. Speaking Together integrates quality improvement techniques with hospital-based language services activities to reduce language barriers faced by patients across America.

In case studies derived from the collaborative, the presentation describes challenges faced by hospitals in delivering care to linguistically diverse populations, presents lessons learned in language services, and identifies preferred practices for effective communication with LEP patients.

Based on what has been learned so far, we know that most hospitals can easily take a few key steps to improve care for LEP patients including: Understand the language needs of the community; Technology is a key driver of patient-centered care; and Communicate and build awareness about language barriers in health care.

Speaking Together: National Language Services Network is a new national program funded by the Robert Wood Johnson Foundation and housed at The George Washington University School of Public Health and Health Services. Speaking Together focuses on improving the quality and availability of language services for patients with limited English proficiency in in-patient and out-patient settings.

\section{0-06 HPV related Cervical Disease Analysis \& Provider \& Parent Perspectives on HPV Vaccine among Immigrants in Washington DC}

Medha Donde (Mary's Center for Maternal and Child Care, Inc)

The HPV vaccine Gardasil is a promising tool in the prevention of HPV related cervical disease. Although health organizations recognize the benefits of the vaccine, concerns remain among parents of adolescent girls regarding its use. Rates of 
cervical cancer in Washington, DC are high at 13.7 per 100,000, compared to national rates at 8.8 per 100,000 .

Latina women in Washington, DC are a population that would benefit from additional cervical cancer prevention techniques such as the HPV vaccine. We analyzed data from Mary's Center, Inc in Washington, DC to determine the burden of HPV related cervical disease among women who receive screening there. Second, we conducted interviews with health care providers and parents of adolescent girls from Mary's Center to determine attitudes and potential approaches to better educate parents on the costs and benefits of the vaccine. Results showed that HPV related cervical disease is a present problem among young women at Mary's Center with $83.1 \%$ of Pap test results showing LGSIL were found in women ages 21-35. Additionally, interviews demonstrated that parents accept the HPV vaccine under the current guidelines but desire to know more about the issue of HPV related cervical disease.

\section{0-07 Experience from the Field: Utilization of Dental Care Services Among Immigrant Taxicab Drivers in Washington, D.C.}

Abmed Elmi, MPH, CHES (Health Educator) (3P Strategies, Inc.)

Purpose: A qualitative study to explore the extent to which immigrant taxicab drivers in Washington, D.C. utilize dental care services and to understand factors that influence utilization of these services. Factors examined included drivers' attitudes toward dental care providers and dental health, the relationship between culture, health behavior, knowledge about preventive dental health, and access to care.

Methodology: Conducted 47 individual interviews with drivers from 10 different countries in Africa and Asia, nine key informant interviews, and two group discussions. Developed structured and unstructured question guides and analyzed the data using open, axial and selective methods of Straus and Corbin. Approval for the study was obtained from the Internal Review Board of the George Washington University Office of Human Research.

Results: Approximately 2/3 of drivers did not have dental insurance or benefits and, those who did obtained it either through their spouse's employment or second job (not taxicab driving). Seventh-five percent of the drivers reported no dental visits in the past year, this included $23 \%$ who had never visited a dental care provider. Of the drivers who visited a dentist within the past year $(25 \%)$, a great majority of them $(84 \%)$ reported a visit due to toothache with the frequent result of tooth extraction. Drivers often sought dental care only when in pain and would resort to over-the-counter pain relievers or home remedies, as their first line of defense to counter pain. Limited knowledge of preventive dental care and inability to pay for services were primary reasons given by drivers for not seeking preventive dental care. Culture and country of origin were not given as reasons for drivers' limited use of dental care services.

Recommendations: Targeted dental health education and access to dental care could help drivers to avoid premature loss of natural teeth, pain, and loss of productivity.

10-08 SAFER Latinos: A Collaborative Intervention Model Addressing Community Mediating Factors for Violence Among Urban, Immigrant Latino Youth

Mark Edberg, PhD; Sean Cleary, PhD; Joanne Klevens, MD, PhD; Elizabeth Collins, MPH; Alex Taylor del Cid, PhD; Luisa Montero; Melba Calderon, MSW (The George Washington University)

In the Washington, DC area, like other communities nationwide, violence among Latino immigrant youth has substantially increased over the past decade. A range of social, demographic and community factors play key roles: patterns of sequential family 
immigration that have consequences for family cohesion and contribute to a reliance on peer socialization; few language and culturally appropriate services for immigrant youth who face school success barriers and drop out early; low awareness/perception of community support; the presence of several major Latino gangs, at least one of which is rooted in El Salvador; and the integration of violence into prevalent youth norms related to status and reputation. A collaboration between the Centers for Disease Control and Prevention (CDC), the George Washington University (GWU) Department of Prevention and Community Health, and two key community partners - the Latino Federation of Greater Washington (LFGW) and the Latin American Youth Center (LAYC), collected baseline data and are implementing/evaluating a tailored intervention called SAFER Latinos (Seguridad, Apoyo, Familia, Educacion, y Recursos) - a primary prevention program targeting community mediating factors for youth violence. This paper will present the intervention model, review baseline data (from a community survey and focus groups), and discuss progress over the first year.

\section{0-09 Reducing Travel-Related Illness among Foreign-Born U.S. Residents Visiting Their Country of Origin}

Erica J Sison, MPH (Newark Department of Health and Human Services); Jeannette Burgos, BS, CHES (Newark Department of Health and Human Services); Yasamin M. Brown, MPH (Newark Department of Health and Human Services); Pauline Thomas, MD (University of Med(Newark Department of Health and Human Services)

Previous research indicates that foreign-born U.S. residents visiting relatives and friends in their native lands perceive less personal risk or threat from travel-related disease than tourists and thus, do not seek healthcare prior to departure. Newark, N.J. has a population of 273,546 , of which $30.0 \%$ are foreign-born $(7.8 \%$ naturalized citizens). Between 2004 and 2006, 89 reportable preventable communicable diseases were linked to travel, including; 28 malaria cases, 19 hepatitis A cases, 9 giardiasis cases, 8 salmonella cases, 7 campylobacteria cases, 5 amoebiases cases, 3 dengue fever cases, 3 shigella cases, 3 typhoid fever cases, 1 brucellosis case, 1 hepatitis B case, 1 leprosy case, and 1 measles case. Destinations included countries in Africa, Asia, Europe, South and Central America, and the Caribbean. We determined that the burden of travel-related preventable communicable diseases warranted intervention by the Newark Department of Health and Human Services. Staff from the Division of Surveillance and Prevention, and Health Education worked together to develop a survey to evaluate local foreign-born residents travel patterns and attitudes, and interventions to promote routine medical care that include counseling and prophylactic measures in preparation for travel abroad. The interventions include: placing travel advisory posters in neighborhood supermarkets, community centers, and religious centers; meeting with community leaders within various ethnic areas; and providing information to health care providers (e.g., grand rounds presentations), faxes and e-mails. We present survey results and the plan for evaluation of the interventions. As global travel and migration continues to increase, public health and healthcare professionals will need to address this emerging health concern.

\section{0-10 Video-Based Intervention on Hygiene for Newly-Arrived Refugees}

Catherine Harbour, MPH (Johns Hopkins Bloomberg School of Public Health); Aisling C. McGuckin RN, MSN, MPH (Baltimore Open Society Community Fellow Baltimore Resettlement Center) (JHU BSPH (CH) and Baltimore Resettlement Center (ACM))

The Baltimore Resettlement Center (BRC) assists newly-arrived refugees. The presentation describes a health education intervention for newly-arrived refugees, 
which is a harm-reduction measure to ameliorate poor quality housing. The centerpiece of the intervention is an entertaining video.

Many refugees are unaccustomed to Baltimore's climate and multi-family housing, and to hygiene practices common in the U.S. Appropriate home hygiene (cleaning, food preparation, and food storage) is essential to preserving household health by preventing cockroaches, mice, etc. Home hygiene also minimizes service needs and maintains good landlord/tenant relationships. Some landlords have expressed frustration at refugees' home hygiene, which can exacerbate existing health threats of low-income urban housing. Personal hygiene is important for obtaining and keeping employment, and for social integration in schools and workplaces. Keeping safe and healthy housing, and keeping a job are important determinants of successful resettlement for refugee families.

The presenters are developing a hygiene intervention for newly-arrived refugees, based around an entertaining video that will be distributed to newly-arrived refugees. The video describes the benefits of good home hygiene and good personal hygiene, the consequences of poor hygiene, and ways to achieve good hygiene practice. The evaluation plan will be described.

\section{SUBSTANCE ABUSE AND MENTAL HEALTH}

\section{1-01 Predictors of Incarceration among Injection Drug Users (IDUs) in Baltimore, Maryland}

Becky L. Genberg; David D. Celentano; David Vlahov; Gregory D. Kirk; Shruti H. Mehta (Johns Hopkins Bloomberg School of Public Health)

Background: In Maryland, $>70 \%$ of incarcerations are attributable to IDU. Understanding the behaviors and characteristics that predispose IDUs to incarceration is critical to informing interventions. We characterized socio-demographic, drug and stability-related predictors of incarceration in a community-based cohort of IDUs in Baltimore, Maryland.

Methods: Semi-annual follow-up visits (1988-2006) from the ALIVE cohort were analyzed. Incarceration was defined as reported jail/prison stays in the prior six months. Logistic regression with generalized estimating equations was used to identify predictors of incarceration; time-varying exposures were lagged one visit.

Results: 2,697 IDUs were followed over a median 10 visits; at baseline, mean age was 36 years, 74\% were male, 93\% African-American, 32\% HIV positive and $84 \%$ were actively injecting. Incarcerations were reported at $14 \%$ of 39,326 visits. In race-adjusted analysis, young age $(\mathrm{OR}=1.89)$, prior incarceration $(\mathrm{OR}=$ 2.26), HIV-positive status $(\mathrm{OR}=1.13)$, higher injection frequency $(\mathrm{OR}=1.72)$, injecting speedball $(\mathrm{OR}=1.42)$, crack use $(\mathrm{OR}=1.16)$, and alcohol use $(\mathrm{OR}=$ $1.24)$ in the past six months predicted incarceration. Stability factors including being in alcohol/drug treatment $(\mathrm{OR}=0.77)$, having housing $(\mathrm{OR}=0.75)$, employment $(\mathrm{OR}=0.78)$, higher income $(\mathrm{OR}=0.50)$, health insurance $(\mathrm{OR}=0.75)$, and a regular provider $(\mathrm{OR}=0.84)$ in the past six months, were protective against incarceration.

Conclusions: While drug-related risk behaviors were strong predictors of incarceration, harm reduction programs that incorporate socioeconomic opportunities, stable housing and healthcare in addition to drug treatment may prevent incarcerations among urban IDUs. 


\section{1-02 Substance Abuse and Mental Health Disorders in HIV- Infected Patients}

Kodjovi Marvis; Afasanwo Benedict Kossi (Action Group on Mental Health $(A G M H))$

Background: Substance abuse (SA) and mental health $(\mathrm{MH})$ disorders are common in HIV-infected $(\mathrm{HIV}+)$ persons and may affect use of any antiretroviral therapy (ART) as well as highly active ART (HAART).

Methods: From the HIV Cost and Services Utilization Study taken in Lagos Nigeria, a population-based probability sample of all HIV+ persons in care in early 2007, we evaluated ART use and type (i.e., monotherapy, HAART, other combination) reported from the first (Jan.- April 2007) . Patient characteristics, SA / drug dependence, and MH disorders were assessed at first FU. In all reported models, we report adjusted odds ratios (AORs) controlling for demographics, lowest CD4 count, and outpatient visits. Results: Of 156 patients in the sample (representing 2267 persons in care), 46\% had SA, 9\% were drug dependent, $38 \%$ had MH disorder, $90 \%$ were on ART, and $61 \%$ on HAART. In separate models for each SA and $\mathrm{MH}$ factor, significantly lower adjusted odds of receiving ART appeared for patients with: drug dependence $(\mathrm{AOR}=.40)$, cocaine use $(\mathrm{AOR}=0.52)$, heroin use $(\mathrm{AOR}=.40)$, sedative abuse $(\mathrm{AOR}=.54)$, amphetamine abuse $(\mathrm{AOR}=.42)$, high SA severity score $(\mathrm{AOR}=.38)$, dysthymia $(\mathrm{AOR}=.74)$, and $\mathrm{MH}$ treatment $(\mathrm{AOR}=.71)$. When both $\mathrm{SA}$ and $\mathrm{MH}$ factors were combined in the same model, drug dependence and severity of drug use were still negatively associated with ART, but $\mathrm{MH}$ variables were not . Among those receiving combination therapy, no SA or MH factors were associated with lower odds of receiving HAART.

Conclusion: The key barriers to ART appear to be drug dependence or high severity abuse, and not $\mathrm{MH}$ disorder, but these factors do not appear to reduce use of HAART once on treatment.

\section{1-03 Mental Health Intervention among Homeless Youths: A Longitudinal Examination}

Sean A. Kidd, PhD; Carrie Bullard, RN, BSc, MN; Kristin Cleverley, RN, BScN, MSc.; Inas Ktaech, B.A. (McMaster Department of Psychiatry and Behavioural Neurosciences Centre for Mountain Health Services - Mental Health Rehabilitation, St. Joseph's Healthcare, Hamilton)

Despite high rates of mental illness and mortality among homeless youths, and the abundance of services across North America working with this population, very little research has longitudinally examined (i) interventions for this group and (ii) risk and resilience trajectories. The present study examines the service utilization and risk and resilience trajectories of homeless youth employing a mixed method longitudinal design. This inquiry included a qualitative examination of youths' experiences of physical and mental health and service access. Youth also completed structured surveys examining past abuse, health and service utilization, current mental health and suicidality, resilience, and the working alliance in their treatment delivered by a mental health nursing service. The mental health nurse, in parallel with the youth surveys, completed a complimentary working alliance inventory for each youth along with measures of community functioning, goal attainment, service utilization and critical incidents. Both youth and mental health nurse measures were completed for each participant up to 3 times with 2-month intervals. Data from the pilot phase of this project are presented including key means through which service utilization was facilitated (e.g., worker availability, increasing awareness of risks) as they were related to measures of risk, resilience, alliance, and community functioning. 
11-04 Injection First: A Unique Group of Injection Drug Users in Tijuana, Mexico

Robin A. Pollini; Remedios Lozada; Kimberly C. Brouwer; Jorge Alvelais; Andrea Mantsios; Steffanie A. Strathdee (UCSD School of Medicine)

Background: Injection drug use is increasing in Tijuana, Mexico, but little is known about circumstances surrounding first injection. We examined injection drug users (IDUs) who undertook injection first, i.e., before using non-injected substances (including alcohol and marijuana) or within their first year of substance use. We hypothesized that these injectors would be more likely to 1) have family members who used/injected drugs, and 2) report riskier behaviors.

Methods: Beginning in 2006, IDUs \&\#8805;18 years old were recruited using respondent driven sampling in Tijuana. Multiple logistic regression identified factors independently associated with injection first.

Results: Of 1,039 IDUs, 88\% were male with median age 35 (IQR: 30-42). Twelve percent met criteria for injection first. Early influences independently associated with injection first were earlier age at first injection $(\mathrm{AOR}=0.81 ; 95 \%$ CI: $0.76,0.85)$ and lower odds of completing primary school $(\mathrm{AOR}=0.61 ; 95 \% \mathrm{CI}$ : $0.38,0.98)$. Interestingly, these IDUs were also less likely to have ever used noninjection substances including alcohol $(\mathrm{AOR}=0.26 ; 95 \% \mathrm{CI}: 0.16,0.43)$, marijuana $(\mathrm{AOR}=0.08 ; 95 \% \mathrm{CI}: 0.04,0.15)$, cocaine $(\mathrm{AOR}=0.45 ; 95 \% \mathrm{CI}: 0.27,0.73)$, and methamphetamine $(\mathrm{AOR}=0.52 ; 95 \% \mathrm{CI}: 0.32,0.84)$; and less likely to have ever overdosed $(\mathrm{AOR}=0.42 ; 95 \% \mathrm{CI}: 0.26,0.69)$ or traded sex for money/drugs $(\mathrm{AOR}=$ 0.36; 95\% CI: 0.18, 0.71).

Conclusions: We identified a unique group of IDUs who began injecting before or shortly following other substance use. Contrary to our hypotheses, these IDUs differed from other IDUs only in their initiation age and schooling, subsequent drug use patterns and tendency toward less risky behaviors. Qualitative research on these IDUs may inform prevention of early injection drug use and high risk behaviors

\section{1-05 Entry into Heroin Addiction Treatment: Implications for Urban Centers}

S. Gwin Mitchell; R. P. Schwartz; S. Kelly; H.S. Reisinger; J.A. Peterson; D. A. Highfield; M. Agar; J.H. Jaffe; B.S. Brown (Friends Research Institute)

Out-of-treatment heroin addicted individuals suffer adverse consequences of addiction and contribute to public health problems in their communities. Despite 40 years of research demonstrating the effectiveness of methadone treatment, in many parts of the world, methadone treatment capacity remains inadequate. Findings from two NIDA-funded studies will be presented which characterize the needs and desires of out-of-treatment heroin-addicted individuals and which address these needs through low-threshold methadone treatment. Data will be presented comparing characteristics of treatment seekers from those who do not seek treatment. Findings will be presented from a recently conducted randomized clinical trial with 319 heroin-addicted individuals seeking methadone treatment who were randomly assigned to interim methadone or to waiting list. Outcomes at 4 and 10 months after study enrollment will be presented in terms of treatment entry, heroin and cocaine use, illegal activity and arrest and HIV-risk behavior. The successful strategy used to sustain to expand this research study into a larger scale clinical service will be discussed. Implications for the health of urban centers will be presented. 


\section{1-06 Substance Abuse And Mental Health In HIV Positive People}

Kodjovi Marvis (Aids prevention initiative nigeria (APIN))

Objectives: This study sought to determine substance abuse and mental health disorders, which are common in HIV infected individuals and may affect the use of any antiretroviral therapy (ART) as well as highly active ART (HAART).

Methods: A total of 57 low-income urban residents receiving mental health and substance abuse treatment who are HIV infected. We evaluate ART use and type (i.e. Monotherapy, HAART, other combinations) reported from first (March-Oct 2006) to second follow up interview (Nov. 2006-April 2007). Patient characteristics, substance abuse/drug dependence and mental health disorders were assessed at first follow up. In all reported models, we report values controlling for demographics, low CD4/Viral load and outpatient visits.

Results: Eighty three patients had a lifetime history of dual disorders, 49 (69\%) had substance abuse, $34(60 \%)$ had mental health disorders, $4(6 \%)$ were drug dependent, $62(84 \%)$ on ART and $56(76 \%)$ HAART. Each disorder was considered primary (i.e. no indication was found that one was caused by the other) for 24 patients in the mental health settings 71 and 31 in the substance abuse treatment setting $63 \%$.

Conclusion: In each type of treatment setting, nearly two-thirds of the patients met criteria for a diagnosis of a dual disorder. Despite these associated disorders, mental health, substance abuse, drug dependence, treatment progresses to the use of the HAART regime once ART commences.

\section{1-07 Improving Mental Health Service Delivery to Hispanics}

Henry Acosta, MA, MSW, LSW (National Resource Center for Hispanic Mental Health)

This presentation will focus on what mental health providers can do in order to improve their mental health service delivery to Hispanics. The session will specifically provide participants with knowledge about the importance of and how to conduct a thorough cultural formulation and social and cultural assessment of Hispanics, especially, Hispanic immigrants. The session will also provide participants with knowledge concerning what professional studies indicate about Hispanics' use of mental health services, barriers in accessing and receiving quality mental health services facing the population, and documented clinical best practices with Hispanics. The session will also focus on the presenter's nationally disseminated and research-driven Model Mental Health Program for Hispanics Report. This report includes recommended steps that mental health agency administrators and direct service providers can take in order to increase their ability to better attract, engage, retain, and serve Hispanics in mental health programs.

\section{1-08 Outcomes Measurements in Substance Abuse: Revealing a Fuller Story Behind the Struggle For Sobriety}

Bernadine Brown (Imani Foundation); Shawn Williams (Imani Foundation) (Social Solutions)

The focus on outcomes in recent years is a welcome trend, but in the field of substance abuse it can also be frustrating. The relapse rate is high, and sole emphasis on the outcome of sobriety can make it appear as if agencies are making little headway.

The Imani Foundation of Atlanta, Georgia, demonstrates how they use performance tracking software to capture data that allows them to tell a richer 
story. Working in an urban setting with a majority of chronically homeless people, often with triple diagnoses, they found that they needed to look beyond sobriety.

It is hard for many consumers to remain in treatment, which in turn makes it difficult to measure progress toward outcomes. Taking a case management approach where the workers start with the consumers most basic needs, the Imani Foundation fosters long-term relationships and increases the likelihood that people will remain in touch with their case worker over time.

The Imani Foundation shares their experiences on the journey, beginning with the frustration in capturing accurate outcomes data, to a place where systematic performance tracking allows them to tell a fuller story about consumers' incremental progress toward outcomes over time.

\section{1-09 Homelessness and HIV Risk Behaviors in Urban Sample of Heterosexuals at High Risk for HIV}

Irene Kuo (Department of Epidemiology and Biostatistics, George Washington University School of Public Health and Health Services, Washington, DC); Manya Magnus (Department of Epidemiology and Biostatistics, George Washington University School of Public H(George Washington University School of Public Health and Health Services)

Background: Homeless individuals are at high risk for HIV infection, but little is known about homelessness and HIV risk behaviors in community-recruited populations.

Methods: Between January-May 2007, 306 sexually-active individuals aged 18-50 were recruited from high-risk urban neighborhoods for the National HIV Behavioral Surveillance system using respondent driven sampling and completed a survey regarding housing, sex/drug use behaviors, and depressive symptoms. Prevalence data were not RDS-adjusted.

Results: Mean age was 37.9 (SD: 10.1); 93.8\% were African-American; 59.4\% were female and $20.6 \%$ were current injection drug users. $74(24.2 \%)$ reported recent homeless (past 12 months), of whom 41 (55.4\%) were currently homeless. Recent homelessness was positively associated with having been in jail (AOR: 2.8; 95\% CI: 1.3-5.6), having exchange partners in past 12 months (AOR: 2.9; 95\% CI:1.5-5.9), and having depressive symptoms (AOR: 2.4 ; 95\% CI: 1.2-4.8) and was negatively associated with having health insurance (AOR: 0.4; 95\% CI: 0.2-0.9). IDU status, having recent unprotected vaginal sex and past HIV testing were not associated with recent homelessness.

Conclusion: Preliminary analyses suggested community-recruited homeless individuals were more likely to experience exposures that put them at risk for HIV infection but less likely to be insured. Interventions to improve support systems for marginally housed individuals are needed.

\section{1-10 Using Technology to Integrate Substance Abuse Screening and Intervention into Medical Care}

A. Rubin; C. Shanahan; E. Cunniff; J. Richardson; K. Pressman; D. P. Alford (Boston University School of Medicine)

More than $8 \%$ of Americans 12 or older have used an illicit substance; about $20 \%$ have had at least one occasion of drinking above recommended limits (SAMHSA, 2006). One of the difficulties in identifying and offering treatment to people with 
unhealthy substance use is that substance abuse screening and treatment are not integrated into the larger medical system. In addition, patients who are at risk or just beginning to experience alcohol or drug problems often are not identified in general healthcare settings. If they are identified, programs are not available to help them. MASBIRT (Massachusetts Screening, Brief Intervention, Referral and Treatment) is one of many SBIRT projects around the country that is attempting to integrate substance abuse screening and treatment into the larger medical system. Our unique approach is to use state of the art technology to accomplish this goal. Using computer telephony (interactive voice response (IVR) and speech recognition) and the Web, we are screening and offering brief intervention treatment to patients before their primary care appointment. With this technology, many more patients can be screened and offered help, and health care professionals can be educated on how to manage these patients.

\section{1-11 The Drug-Free Workplace Kit: An Interactive Workplace Prevention and Early Intervention Web Site}

Robert L. Stephenson, MPH SAMHSA/CSAP; Deborah M. Galvin, PhD SAMHSA/ CSAP; Alan Bekelman, (President, DSG); Thomas Vischi, MA, MCP, PhD, (DSG), Carolyn Lorente, PhD (DSG) (Development Services Group, Inc.)

The National Survey on Drug Use and Health indicated that most heavy drinkers and illicit drug users are employed. Substance abuse among workers is associated with a variety of negative outcomes. Thus, the workplace could be a fruitful area for delivering opportunities for preventing substance abuse.

Several studies have indicated that successful drug prevention strategies for workplaces integrate substance abuse prevention and early intervention within broader health and wellness programs. Further, these programs have an excellent return on investment ranging from $\$ 5$ to $\$ 30$ on the dollar. Workplace health programs allow employers to reach large numbers of employees and their families at a low cost; reduce absenteeism, and the stigma of substance abuse, while enhancing confidentiality, productivity, and employee morale.

Past analysis of the GetFit.SAMHSA.Gov interactive health/wellness workplace website increased knowledge related to substance abuse while successfully reaching employees needing early intervention. The new Workplace Kit, which has embedded GetFit within its structure, is hypothesized to provide a high return on investment for large to small businesses. This presentation will provide an overview of the Kit (e.g., conceptualization, design, use, and effectiveness) and some early process findings related to early adapters of the Kit. The workplace kit is easily adaptable to a variety of workplaces (small to large, public/private, diverse populations, industries and occupations).

\section{1-12 Diabetes Risk and Prevalence among Drug Users in New Jersey}

Milton Mino; Sherry Deren, PhD; Sung-Yeon Kang, PhD (NATIONAL DEVELOPMENT AND RESEARCH INSTITUTES)

Background: Drug users are at high risk of developing debilitating health conditions. This study presents preliminary data on the risk for diabetes among drug users in New Jersey.

Methods: Participants $(n=80)$ were recruited from 2 NJ urban methadone $(\mathrm{MM})$ clinics or were current heroin and/or cocaine users. Subjects were recruited 
from an HIV intervention study on migrant Puerto Rican drug users and used heroin or cocaine in P.R. or had a friend/family member who had.

Results: Participants had a mean age of 40 and $70 \%$ were male. Based on their body mass index, $39 \%$ were overweight, $19 \%$ obese and 4\% extremely obese. $53 \%$ got little or no exercise. $19 \%$ had a sibling and $42 \%$ had a parent with diabetes. Despite these diabetes co-factors, only $4 \%$ had a diabetes diagnosis. In a subset of participants $(n=9)$ who took part in a health training, 8 said they would attend a longer diabetes training at their MM clinic if offered.

Conclusions: Drug users and their networks have a high prevalence of diabetes risk factors. The very low self-reported rate of diabetes likely reflects undiagnosed cases. MM clinics can be effective settings to provide diabetes related screening and education to this population.

\section{1-13 Drinking in Context: Exploing the Relationship among Gender, Neighborhood Deprivation and Alcohol Consumption}

Flora I. Matheson; Rahim Moineddin; James R. Dunn; Heather White; Richard H. Glazier (Centre for Research on Inner City Health, St. Michael's Hospital)

This presentation will explore relationships among gender, neighborhood material deprivation and patterns of alcohol consumption in a large national sample of adults living in 25 of the largest urban areas in Canada $(\mathrm{N} \sim 70,000)$. Using the Canadian Community Health Survey in conjunction with tract-level data from the 2001 census preliminary results suggest that neighborhood material deprivation is associated with higher alcohol consumption. A significant random intercept in the multilevel regression models suggest that patterns of alcohol consumption vary across neighborhoods and a significant cross-level interaction among gender and material deprivation suggests that the relationship between neighborhood material deprivation and alcohol consumption is most pronounced among men. Finally, in the presentation we will explore the impact of health and behavioral risk factors on this relationship.

\section{1-14 The Influence of Social Capital on Substance Use-Related Health Behaviours} Maritt Kirst (University of Toronto, Department of Sociology)

Substance users such as injection drug users and crack smokers are at risk for several health problems, including blood-borne disease infections such as HIV, Hepatitis B and $\mathrm{C}$, and overdose. Behaviours that contribute to health risk stem not only from an individual's knowledge and beliefs, but are also shaped by processes of influence and constraint operating within networks of social relationships. The analysis of users social network relationships is therefore important to understand users engagement in drug use-related risk behaviours. Such an analysis produces knowledge of the social contextual nature of risk decision-making that is useful to assist in breaking down barriers to risk reduction within drug use settings. This presentation will discuss findings from a research study that has explored the influence of social network-based resources, or social capital, on drug use-related risk and protective behaviours among a sample of 80 injection drug users and crack smokers in the urban centre of Toronto, Canada. This presentation will discuss how aspects of social capital that contribute to and/or reduce risk within the context of drug use may be harnessed within harm reduction services to help users minimize health risks within drug use settings. 


\section{URBAN HEALTH FROM A GLOBAL PERSPECTIVE}

\section{2-01 Implementation of the Medical Undergraduate Urban Health Programme at Universiti Teknologi Mara (Uitm), Malaysia}

A. D. S. Krishnapillai; A. S. Ramli; A. Suleiman; M. Mokbtar; K. Yusoff, Faculty of Medicine, Universiti Teknologi MARA, 40450 Shah Alam, Selangor, MALAYSIA (UITM, SHAH ALAM)

Introduction: As urbanization grows right across the globe, Malaysia is no exception to this major demographic shift. The Lancet bemoaned that most medical schools had neglected teaching Urban Health and to date, only rural health curriculum has been addressed in many medical schools across Malaysia. UiTM has taken a step forward to develop an undergraduate Urban Health programme to equip our students with this new paradigm.

Objectives: This paper aims to describe the implementation process of Urban Health curriculum at UiTM, its challenges and future directions.

Implementation methods: An Urban Health curriculum development workshop was carried out. Various experts from different specialties and local authorities were invited. Four major components were identified and emphasized in the curriculum - the health delivery system in urban areas, physical component, psychological component and social aspects. The programme is a 4-week posting implemented in Year 3 of the 5year medical undergraduate course. It encompasses of lectures on various urban issues, seminars, field visits to various NGO's in urban areas, written report on field visits and a comprehensive urban home visit with case write-up and presentation.

Discussion and Conclusion: The experience in UiTM can provide a possible model of an Urban Health programme being implemented in undergraduate medical education. Given the major demographic shift, there is no doubt that Urban Health should be taught to prepare our students before they are entrusted with the burden of managing health in urban areas.

Key words: Urban health, Medical education, Undergraduate curriculum, Implementation, Malaysia

\section{2-02 PAH0's Approach Addressing Social Determinantes and Equity in Urban Settings of the Americas}

Eduardo Guerro Espinel; Marilyn Rice; Carlos Castillo-Salgado (Pan American Health Organization)

The Americas, like other developing regions of the world, is going through a process of massive urbanization in a context of high poverty levels. As such, the region's cities are facing health challenges particular to this rapid urban growth. These challenges include a dual burden of both infectious and chronic diseases and marked social inequities, which promote high levels of interpersonal violence and of mental health problems. Furthermore, the cities face high traffic-related accidents and inadequate public services provision.

$\mathrm{PAHO}$, in an effort to provide technical assistance on urban health (UH) to the Region's countries and cities, organized a Regional Forum in July 2007 in Mexico City. Aimed at addressing the challenges of rapid urbanization, this forum had three goals. The first one was to close the knowledge gap concerning UH in the Region. The second was to propose a theoretical and an operational framework for UH to be used by PAHO. And, the third was to create two working groups, one on the measurement of $\mathrm{UH}$ (including the development of new indicators), and one dedicated to UH 
governance (including policy and decision making). Both working groups have continued to work on their specific topics.

During the ICUH 2007, PAHO will present a summary of the working documents produced by the Regional Forum and the products of the two working groups.

12-03 Empower the Community, Equip the Providers: Creating Successful CommunityFacility Referral Linkages for HIV and Reproductive Health in the Urban Slums of Nairobi, Kenya

Jane Otai (JHPIEGO/Kenya); Dr. Pamela Lynam (JHPIEGO/Kenya); Dr. Daniel Nguku (Medical Officer of Health-Nairobi City Council); Adan Golo (Secretary of Korogocho Village Health Committee); Moses Nyayiemi (Chairman of Viwandani Village Health Committee) (JHPIEGO-an affiliate of The Johns Hopkins University)

KEY HEALTH ISSUES:

HIV and reproductive health in urban slums

Comprehensive Care for HIV in urban slums

Partnership approaches to impacting urban community health

\section{LEARNING OBJECTIVES:}

Discuss the unique community and facility health needs in Africa's urban slums

Discuss a methodology that includes both communities and facility-based health providers to improve the quality of urban health services through demand generation and community responsiveness

Demonstrate how PQI and similar approaches bring urban clinics and urban communities together.

The exponential growth of urban slums in Africa is set to continue unabated. This has created communities that are not educated about health and are served by only a few basic health facilities which are overburdened, under trained, and ill-equipped. To address these needs, JHPIEGO has instituted a comprehensive care program in two slums in Nairobi to strengthen linkages between health clinics and community organizations and to ensure that community members have access to quality health services. By using the Performance and Quality Improvement (PQI) process - a self assessment that enables health providers and communities to identify gaps, and then develop plans to fill those gaps - providers and community leaders have: (i) increased demand for quality health services and responded to that demand accordingly; (ii) strengthened linkages among community groups and clinics (the community now talks of our clinic); (iii) created greater respect for community by clinic staff and vice-versa; (iv) improved the quality of services through better case management at the facilities and more informed expectations among community members; and (v) met the identified health needs of the community by creating urban gardens in clinic waste spaces, worked with support groups for HIV and rape victims, among other interventions.

12-04 Quality of Care and Other Predictors of Women's Use of Private and Public Facilities for Skilled Birth Attendance and Satisfaction, in Informal Settlements of Nairobi, Kenya

Eva S. Bazant; Michael Koenig; Jean-Christophe Fotso (Johns Hopkins Bloomberg School of Public Health)

Maternal mortality claims over 500,000 women's lives globally each year. In SubSaharan Africa, even among urban dwellers, many women still deliver at home, without skilled birth attendance. Access to and quality of health care is often 
lacking. This study quantifies reports of women in two slums of Nairobi, Kenya on quality of maternal health care in public and private facilities; highlights individual and service-related predictors of use of both facility types for skilled attendance; and predictors of satisfaction.

In 2006, a household survey on maternal health was carried out with 1,926 mothers. We entered 15 items with Likert response-scales into exploratory and confirmatory factor analyses, and used multivariate multinomial regression models.

A third of women delivered at home or with a traditional attendant, $44 \%$ delivered in private facilities, and $22 \%$ at government facilities. Nearly all attended prenatal care. Approximately $75 \%$ of respondents agreed with positively-worded items regarding quality, but this varied by item between private and public facilities.

Predictors of birth in either facility type were women's area of residence, parity, ethnic group, wealth, autonomy, and husband's education. Prenatal care aspects were also significant, such as quality experienced at public facilities, being advised to deliver in a facility, number of visits, and costs. Quality counseling in prenatal care, women's education, and having an obstetric complication was associated with delivery in a public facility. Women's area of residence, pregnancy intendedness, and prenatal care facility type was associated with delivery in a private facility. Women's satisfaction with the care was strongly associated with the delivery care quality (empathy and counseling) received.

To increase skilled birth attendance, encourage use among underrepresented groups, improve interpersonal and clinical quality, access to care, and reduce unintended pregnancies.

\section{2-05 National Institute on Drug Abuse (NIDA) International Program (IP) Research Training and Exchange Programs}

E. S. John (IQ Solutions, Rockville, MD, USA); S. W. Gust (National Institute on Drug Abuse, Rockville, MD, USA); E. L. Winstanley (Behavioral Pharmacology Research Unit, Johns Hopkins School of Medicine, Baltimore, MD, USA) (IQ Solutions)

AIM: Describe the research training and exchange programs supported by the International Program (IP) at the National Institute on Drug Abuse (NIDA), the countries and institutions represented by Fellows and Mentors, and Fellows' evaluations of the programs. BACKGROUND: Three programs foster international collaboration on drug abuse research: (1) The NIDA Hubert H. Humphrey Drug Abuse Research Fellowship provides mentored academic training and a research affiliation with a NIDA-supported scientist for mid-career drug abuse professionals from low- and middle- income countries. NIDA Humphrey Fellows learn about NIDA-supported drug abuse research and the application of research to the development of science-based prevention programs, treatment protocols, and government policies. (2) The INVEST Drug Abuse Research Fellowship provides rigorous postdoctoral research training with a NIDA grantee. INVEST Fellows also attend NIDA orientations and participate in scientific meetings. (3) The Distinguished International Scientist Collaboration Program supports 1- to 3-month research exchange visits for senior drug abuse scientists from other countries and their NIDA-supported colleagues. RESULTS: Between 1990 and 2006, 107 Fellows were funded through the NIDA IP training and exchange programs. The Fellows represent 47 countries, $71.96 \%$ of which were low- or middle- income countries. The Fellows' Mentors represent 52 institutions from 19 U.S. states. 


\section{CULTURAL AND ETHNIC DIVERSITY IN URBAN HEALTH}

13-01 Utilizing Critical Incident Technique (CIT) for Exploring Disparities in Breast Cancer Care and the Healthcare Decision Making Process

Michael A. Yonas, DrPh; Robert Aronson, PhD; Nora Jones; Nettie Coad; Eugenia Eng, DrPH; Dinushika Mohattige, MPH(c); Viola Monroe; Brandoyn White, MPH; Christina Hardy, MPH (Department of Health Behavior and Health Education, University of North Carolina at Chapel Hill)

The Cancer Care and Racial Equity Study (CCARES) is an NIH funded CBPR project which utilizes principles of undoing racism to understand factors contributing to racial disparities in breast cancer care. This presentation will describe the methods used and preliminary findings from an innovative qualitative interviewing approach called Critical Incident Technique (CIT) to learn about factors that influence the healthcare decision making process. Each woman in the study is asked to participate in three separate critical incident interviews, each interview focusing specifically on a different phase of the cancer treatment process: the diagnosis phase, treatment, and follow-up phases of care. The aim of using this technique is to assist participants in describing specific incidents from memory, focusing on detail and experience which in this case is the treatment course for women with breast cancer. Our long-term goal is to use knowledge gained from CCARES to eliminate breast cancer care and outcome disparities through the design and evaluation of an intervention that will: (a) prevent deviations from reasonable breast cancer care; (b) monitor and prevent discontinuation of breast cancer treatment and care; and (c) ensure accountability from the healthcare community for adopting new standards/ protocols for breast cancer treatment and care.

13-02 The Sociocultural Context of Newport Brand Preference among Young Adult Urban African Americans Living in Inner City Baltimore

Simona C. Kwon, DrPH, MPH; Katherine Clegg-Smith, PhD; Frances A. Stillman, EdD; Lee Bone, MPH; Emmanuel Price; Norman Yancey (Johns Hopkins University, Bloomberg School of Public Health)

Purpose: Ideas and attitudes about tobacco use are often shaped by socio-cultural values. Such beliefs likely influence decisions to smoke as well as brand choice. Previous research of a community sample of urban young adult (18-24 years of age) African Americans enrolled in work-education training programs in Baltimore found that $62 \%$ smoke and $96 \%$ smoke Newport brand cigarettes. The project aim was to use a community-based participatory research (CBPR) approach to understand the socio-cultural meaning of smoking mentholated cigarettes (i.e., Newport) for this group.

Methods: Using focus groups $(\mathrm{N}=29)$ and in-depth interviews $(\mathrm{N}=7)$, a volunteer, convenience sample of urban, young adult African Americans enrolled in a work-education training program were interviewed.

Results: Newport was identified as the only cigarette brand smoked by the group and the larger urban African American community in Baltimore. Little brand experimentation beyond Newport was reported. Smoking Newport was described as part of their culture and even hereditary. The majority also cited its wide availability in the community from the ease of sharing and bumming to the convenience of buying loosies on the street.

Implications: Because of the dominance of Newport cigarettes, interventions to reduce smoking in this group must address community norms and brand preference. 


\section{3-03 Influences on Food Purchasing Decision-Making among Low-Income African-American Women in Chicago: A Qualitative Study}

Shannon N. Zenk, PhD; Angela M. Odoms-Young, PhD; Constance Dallas, PhD (University of Illinois at Chicago)

Background. The purpose of this study is to examine influences on food purchasing decision-making among African-American women residing in low-income urban communities with limited food availability (e.g., no major supermarket).

Methods. We are conducting in-depth, semi-structured interviews with AfricanAmerican women ages 21-45, recruited from a neighborhood health center in Chicago, in this ongoing qualitative study (18 of planned 30 interviews completed). Constant-comparison and GIS are being used to identify themes and map and measure distances to stores where women shopped.

Findings. Initial findings indicate that in spite of numerous structural barriers and limited resources women devised strategies to access food outlets and products. They often bypassed neighborhood stores and traveled relatively long distances to reach stores perceived as desirable, despite few owning automobiles. They also deliberately shopped at multiple stores to maximize quality and minimize costs. Data analysis revealed three major categories of influence on food purchasing decision-making: (a) individual/household factors (economic, physical, social, personal); (b) food outlet characteristics (physical, owner/employee characteristics and practices); and (c) food product characteristics (variety, quality, price, presentation).

Conclusion. Women negotiated distance barriers to access desirable food outlets and products. More work is needed to identify individual- and policy-level solutions to assist urban African-American women.

\section{3-04 Barriers to Medical Services: One Step Beyond the Linguistic Paradigm}

Suzanne Guy; Sarah Hykes; Katie Nahas; P. Rafael Hernýndez-Arias (DePaul University)

Objective: The broad objective of this study is to assess the health assets and needs in four Chicago communities. The specific objectives of the interviews are to identify: community trends, the social context of health, illness and medicine, and perceptions on availability and access to medical services.

Methods: This paper is based on 27 in-depth individual interviews with key informants from the four communities. The interviews were guided by a structured discussion guide and lasted approximately forty-five minutes. We transcribed the interviews and analyzed them using Atlas-TI. We developed patterns from the development of codes, conceptual families, and conceptual networks.

Findings: Respondents vividly describe the substantial population increased in the four communities during the past fifteen years, due primarily to immigrants. Most respondents positively described population diversity, but lamented the existing economic diversity. Some respondents were able to describe the health needs and assets of immigrant. Fewer respondents could describe the medical needs, access and utilization of medical services among immigrants. Respondents explained that the lack of knowledge about health needs, assets and utilization is due to cultural, primarily linguistic-based barriers. Respondents indicated that linguisticbased fears as prominent among immigrants, such as embarrassment about limited language abilities.

Conclusions: Language is often cited in the public health literature as an important barrier to accessing social and medical services. Less attention has been placed on the emotional components related to language. Linguistic-based fears are 
not solely related to knowing English, but also to the emotions involved in social interactions. Understanding the emotional processes linked to language and other cultural expressions might reveal the elements that form the barrier to accessing social and medical services.

\section{3-05 Stakeholder Attitudes Towards and Feasibility of Interventions to Reduce Drug- related Harms in Tijuana, Mexico}

Morgan Philbin; Andrea Mantsios; Remedios Lozada; Robin A. Pollini; Jorge Alvelais; Patricia Case; Carl A. Latkin; Tim Rhodes; Steffanie A. Strathdee (Proyecto El Cuete Team) (University California At San Diego)

Injection drug use is a growing urban health crisis along the U.S. - Mexican border and rising rates of blood-borne infections highlight the pressing need for harm reduction interventions. We explored the acceptability and feasibility of such interventions in Tijuana, a city adjacent to San Diego, CA. In-depth qualitative interviews were conducted with 40 key stakeholders pharmacists, legal professionals, health officials, drug treatment providers, and police officers to assess their attitudes and practices. Topics included acceptance and feasibility of needle exchange programs (NEPs), syringe vending machines and safer injection facilities (SIFs), barriers, and structural limitations. Interviews were taped with consent, transcribed verbatim, and translated. Content analysis was conducted to identify themes. Eighty percent (29/37) supported at least one intervention, with most support for NEPs (28/36). Only half believed these interventions could be implemented in Tijuana's social/political context, due to barriers involving religion, police, culture, lack of political will, awareness, and funding. Suggestions to facilitate future implementation include using media outlets and trusted figures (church officials/teachers) to increase general community knowledge regarding drug use and associated diseases; raising awareness among politicians by providing costeffectiveness data; documenting the success of Mexico's existing NEPs; and changing local laws to allow program piloting. This research indicates an urgent need to 1) raise awareness and 2) act upon identified steps for developing socialstructural interventions to create enabling environments that facilitate implementation of harm reduction interventions in Tijuana.

\section{OTHER}

14-01 Availability and Cost of Healthy Fresh Food Choices in 36 New York City Neighborhoods Danielle C. Ompad; Katherine R. Standish; Vijay Nandi; Sandro Galea; John R. Beard; David Vlahov (New York Academy of Medicine, Center for Urban Epidemiologic Studies) Access to affordable, healthy, fresh food is an important component of healthy living. We conducted a food store survey in 36 ethnographically-defined neighborhoods in New York City. The residents in the target neighborhoods are predominately black $(57.7 \%)$ and Hispanic $(46.9 \%)$ and experienced significant socioeconomic disadvantage; the median annual income was $\$ 22,438,40.1 \%$ lived below the poverty level and $45.7 \%$ of adults $>25$ years did not have a high school diploma or equivalent (source: US Census 2000). All food stores (e.g., corner stores/ bodegas, grocery stores and supermarkets) in the target neighborhoods were enumerated and a stratified random sample of one of each type of store was selected for each neighborhood. The survey evaluated availability and cost of more than 60 food and grocery items. We identified 425 food stores (181 corner stores/ 
bodegas, 198 grocery stores, and 46 supermarkets) in the sampling frame; there were more corner stores/bodegas (mean 5/neighborhood) and grocery stores (mean 5.5/ neighborhood) as compared to supermarkets (mean $1.3 /$ neighborhood) in the neighborhoods. 106 stores were selected for the survey which included items on availability and cost of selected fresh food items [e.g., milk (whole and low fat/skim), yogurt (whole and low fat), eggs, fruit (grapes, apples, oranges, lemons/limes), vegetables (potatoes, onions, carrots, lettuce, broccoli, tomatoes)]. Corner stores/ bodegas and grocery stores were significantly less likely to have reduced fat or skim milk, low fat yogurt and fresh fruits and vegetables compared to supermarkets. There were few differences with respect to cost: whole milk, reduced fat or skim milk, and low fat yogurt were more expensive in corner stores/bodegas and grocery stores compared to supermarkets. The scarcity of full service supermarkets means limited availability of healthy fresh food choices in these neighborhoods of concentrated disadvantage.

\section{4-02 Going Global: Extending the Health Center Mission through Partnerships with Developing Nations.}

\section{Shira Gitomer (National Association of Community Health Centers)}

The national network of health centers continues to provide high quality, cost-effective, accessible care, serving nearly 8 million urban patients, yet many ask ý what more can we do? In response to the unending need to provide greater access to primary and preventive care, several health centers have begun working globally with communities, healthcare advocacy groups, and academic centers with a common goal to share and spread the health center model to those most in need. This session will highlight the work being done nationally and internationally at the community level and the role others can play in helping to promote the well-being of the underserved population.

\section{URBAN DISASTERS}

\section{5-01 Surveillance for Heat-Related Illness in New York City: A Retrospective Analysis of the 2006 Heat Wave}

Kristina B. Metzger; Jingsong Lu; Kevin Konty; Thomas Matte (NYC Dept of Health and Mental Hygiene)

In 2006, New York City experienced a severe 10-day heat wave during which 46 people died of heat stroke. We conducted a systematic retrospective analysis of realtime syndromic surveillance data, including daily counts of heat-related emergency department (ED) visits and EMS calls in order to evaluate the usefulness of these data in assessing the health impact of an ongoing heat wave ( $\& \# 8805 ; 3$ days at \&\#8805;90degrees F). Two models using Poisson regression (one controlling for temporal trends only, the other controlling for temporal trends and meteorologic factors) were developed using data from May-September, 2002-2005 (ED visits) and 1999-2005 (EMS calls). A simulation of daily analyses was then conducted retrospectively for summer 2006, which included 25 hot ( $\& \# 8805 ; 90$ degrees F) days and three heat waves (17 days total). We observed an excess of heat-related ED visits and EMS calls given the time of year and weather conditions (signal) on 4 days and 8 days, respectively, all on hot days occurring during heat waves. No signals occurred during the first heat wave (7/2-7/4); EMS signals occurred on the first two days of the second heat wave (7/16-7/19). During the third and most severe heat wave (7/27-8/5), ED and EMS signals were first observed on $7 / 30$ and $7 / 29$, respectively. These signals occurred several days before the first heat stroke deaths were reported on $8 / 4$, suggesting that prospective surveillance of heat-related illness 
could be used to augment public health messages about the severe health effects of an ongoing heat wave. Based on these analyses, we have established a protocol for conducting surveillance of heat-related illness during summer 2007.

\section{5-02 Disaster Risk Reduction}

\section{Dr. Jostacio Moreno Lapitan (WHO Centre for Health Development)}

Consistent with the urban disaster risk reduction framework-technology called the Hyogo Framework for Action (HFA) 2005-2015: Building the resilience of nations and communities to disasters which explicitly states that there is a need to: 1) integrate disaster risk reduction planning into the health sector; 2) promote the goal of hospitals safe from disaster; and 3) implement mitigation measures to reinforce existing health facilities, particularly those providing primary health care, a World Health Organization (WHO) consultation workshop on coordinated work for disaster risk reduction and the preparedness of health facilities in urban settings was convened on 18-20 April 2007 in Kobe, Japan. The consultation workshop included multidisciplinary experts and representatives of governmental and non-governmental organizations. It reviewed lessons learnt, policies and programmes, methodologies, sound practices, needs and capacities on disaster risk reduction and the preparedness of health facilities; identified challenges and problems as well as opportunities and solutions; and outlined recommendations for taking the coordination work forward at the global, country and city levels. The ICUH presentation will provide a detailed discussion of the disaster risk reduction issues addressed with a focus on 5 priority actions: 1 ) political will; 2) risk identification; 3) knowledge management and education; 4)risk management; and 5) preparedness for effective response. It will highlight coordinated work for a broad health facility mindset-technology that will help reduce urban disaster risks, improve urban health and bring about behavioral and policy changes.

\section{5-03 The Emergency Action Plan: Regulating High Rise Safety}

Robyn R.M. Gershon, MHS, DrPH; Melissa Wong, MPH (Columbia University)

Introduction: In 2006, NYC passed a rule that affected high-rise business occupancies, entitled "Office Building Emergency Action Plans" (3RCNY 6-02). This new rule established procedures and requirements for the orderly evacuation of occupants from office or group $\mathrm{E}$ buildings due to numerous types of natural or man-made disasters.

The Emergency Action Plan (EAP) must include provisions for the following: shelter-in-place, in-building relocation, partial or full evacuation, as a result of different emergencies occurring within the building or in the vicinity.

A key element of the plan is the certification of the Emergency Action Plan Director (EAPD) (3 RCNY 9-08), entitled, "Training Courses for Fire Safety/ Emergency Action Plan Directors".

Methods: EAPDs receive special training and must be certified before they can assume EAPD responsibilities. Training is provided through 7-hours of classroom instruction.

Results: To date, 509 individuals completed training and 445 passed the exam. EAPDs are now preparing their buildings for on-site exams after which their buildings will be fully compliant with local law 6-02.

Conclusion: Training and education of EAPDs is a feasible approach to preparing high-rise occupancies for response to numerous disasters. This rule provides guidance to other cities concerned about the safe and effective evacuation of high-rise occupants. 


\section{5-04 Web-based WMD Training for Urban Mass Transit Police}

Robyn R.M. Gershon, MHS, DrPH; Melissa Wong; Charles DiMaggio, PhD; Robert Terrett; Dario Gonzalez, MD, FACEP (Columbia University)

Introduction: Transit police are critical for an effective response to a transit-related terrorist attack. Recent events at train stations underscore their potential vulnerability and difficulty for an effective response. While training and education of first responders are crucial, training designed for transit police is limited.

Methods: A state-of-the-art web-based simulation training program was developed for transit police officers.

Results: In addition to a basic WMD education module (agent categories, awareness and response, crowd/perimeter control and mass evacuation from urban transit hubs) three additional simulated modules were developed to address the transit police response to a bioterrorist, chemical or dirty bomb attack. Pre/post tests (addressing six major constructs: knowledge, attitudes, beliefs, influence of subjective norms, intentions to respond and barriers to respond safely) assessed effectiveness. 150-200 transit police will be recruited to complete the training modules and complete the assessment tools.

The training modules and assessment results will be presented. Guidance for using the modules or adapting them to specific transit settings will be provided.

Conclusion: This program represents a unique technological approach by providing training and education to a critical response group in the urban setting.

The use of simulated training techniques is a novel method of disaster preparedness training.

\section{5-05 Responding to Responders: Has the Federal Government Answered the Needs of 9/11 Recovery Workers}

Yvonne Bokhour, Master's Candidate (Health Advocacy Program, Sarah Lawrence College); Peter S. Arno, PhD (Professor of Health Economics, Health Advocacy Program, Sarah Lawrence College) (Health Advocacy Program, Sarah Lawrence College)

Introduction: After the World Trade Center disaster on September 11, 2001, approximately 40,000 recovery workers were exposed to a wide range of hazardous materials. Disabling, even fatal, health problems ensued. This study examines the federal government's response to workers' medical issues.

Methods: We analyzed publicly available documents including Senate hearing testimony, government reports, bills under consideration, laws passed, and commentary by legislators, scientists, victims and other stakeholders.

Findings: The allocation and distribution of health monitoring and treatment funds has been slow, complicated by political wrangling, bureaucratic complexity and mismanagement. A variety of public and private programs were eventually instituted serving different populations, but care has been inconsistent. Until recently, these programs were designed to track health issues, not treat individuals. A long-term, comprehensive plan to monitor and treat has not yet been established.

Conclusion: A federal, coordinated response would have increased efficiency and reduced hardships after 9/11. New York was fortunate to have a relatively wellrun health system, along with outspoken advocates, but it has suffered because of inadequate federal plans and resources. An integrated federal response is needed in order to promote effective, humane care for responders' considerable long-term healthcare needs and to develop plans for future disasters. 


\section{URBAN HEALTH AND GIS}

16-01 The Role of Geography in Access to Medicaid Primary Care: A Spatial Examination of Access in Philadelphia County

Heather A. Klusaritz, MSW; Nadia Corbin, MSW (School of Social Policy \& Practice, University of Pennsylvania)

Purpose: Uninsurance rates reached over 45 million in 2006. While, Medicaid expansions propose to expand coverage to low income groups, access to care issues remain. Provider preferences for practice location and Medicaid participation create a dearth of primary care providers (PCP) in impoverished communities. This paper examines the spatial relationship between communities of high Medicaid recipient concentration and PCP practice location.

Methods: Using ArcView GIS, Medicaid participating PCPs, community health centers, and emergency departments (EDs) were geocoded and mapped in an urban county. Census data for poverty and African American population rate served as proxies for high Medicaid recipient concentration. Availability of PCPs within 2-and 5 -mile radii of census block centroids was examined.

Results: Census block groups with high African American population rates lacked PCPs. Additionally, health centers and EDs were identified as primary access points within a 5 -mile radius of these communities. No discernable pattern exists between census block poverty and the location of Medicaid-participating PCPs.

Implications: The literature documents a strong relationship between access to care and health outcomes. This study indicates spatial access barriers for Medicaid consumers. Eligibility expansions must encourage PCP Medicaid participation and practice location for communities with a concentration of Medicaid consumers.

\section{6-02 The Spatial Association Between the Location of Injury and Land Uses}

M. Cusimano; S. Marshall; M. Chipman; C. Rinner; R. Glazier; T. Hernandez; K. Jones; R. Burgess; T. Bekele (Injury Prevention Research Office, St. Michael's Hospital)

Objective: The purpose of this study was to describe the location of intentional and unintentional injury for four specific age groups (0 to 14, 25 to 24,25 to 64 , and 65 and over) and link the geographic patterns encountered with land use and environmental factors.

Methods: This study utilizes the database of the Toronto Emergency Medical Services for the study of the location of serious injury. Variables include patient age and gender, type of call, and time and date, as well as dispatch location, geocoded to the address or intersection of where the incident occurred. The four types of injury mechanism examined are falls, motor vehicle or traffic-related, assaults and selfinflicted injuries. Using the Arc GIS package, data is presented on maps using the kernel density function.

Results: There are significant differences in the geographic patterns of injury location based on injury type and age group. Many of these patterns can be explained by land use characteristics such as the locations of night clubs and bars, traffic patterns and local socioeconomic characteristics.

Conclusions: After conducting a broad literature search, this is likely the first time in which ambulance data has been used to examine the geographic location of injury, and was found to be a valuable tool. The locations of injury are associated closely with land uses and vary according to injury type and age. 


\section{6-03 Neighborhood Factors Associated with Asthma among Urban Adolescents}

Sara L. Gale; John D. Radke; Adam Davis; Ira B. Tager (University of California, Berkeley)

Despite promulgation of national and international guidelines on the effective management of pediatric asthma, asthma-related morbidity among urban children remains disproportionately high. Although previous research has demonstrated that asthma-related morbidity in urban communities has both strong social environmental and physical environmental antecedents, these two domains are infrequently considered jointly in analyses. Further, asthma case identification is often conducted with health utilization data, which do not reflect the full spectrum of asthma severity in the community. In order to address these limitations, a geographic information system (GIS) was used in conjunction with a community-based asthma survey to determine the relation between the distribution of asthma severity and neighborhood physical and sociodemographic attributes for an adolescent population in Oakland, CA. Asthma severity was ascertained by a school-based case-identification survey for sixth grade students in Oakland public schools during Fall 2006. Students with valid residential address data $(n=1,530)$ were geocoded to an Oakland census tract $(n=106)$, a proxy for neighborhood. Neighborhood-level exposure variables included physical environmental attributes (land use patterns, public and assisted housing facilities, closed-access roadways) collected from state and local planning commissions, as well as demographic and economic attributes from the 2000 Census. Generalized estimating equations (GEE) were used to account for correlation of children who resided in the same neighborhood. Controlling for individual-level covariates, percent of foreign-born census tract residents was the only neighborhood variable associated significantly with asthma case status (OR: 0.957, 95\% CI: 0.919, 0.998).

\section{DEMOGRAPHICS: AGING/GENDER/ETHNICITY}

\section{7-01 Prevalence and Patterns of Gender-Based Violence amongst Market Women in Ibadan Metropolis}

Akpeh Nnabuchi; Erim Daniel; Otite Oliver; Menakaya Chinyere; Odewunmi Sola; Adeniji Adedeji; Dawodu Oluwaseun (Critical Peers)

Gender-based violence is a major public health and human rights problem throughout the world especially in urban areas. However lack of reliable data on the root cause, magnitude and consequences of the problem has been a major obstacle in the search for solutions. The authors did a cross-sectional study in which they obtained data from 635 interviews with market women spread across 3 major markets with Ibadan Metropolis in June 2005. Analysis of this data showed that all respondents had faced one form of Gender-based violence either in their childhood, adolescence or reproductive age group: forced to hawk goods while their male siblings attended school (39\%); sexually abused as children $(9.8 \%)$; forced first sexual intercourse $(9 \%)$; female genital mutilation $(58.4 \%)$; denial of education on basis of sex $(25.6 \%)$; battering by their male partners $(16.8 \%)$; rape by partner $(9 \%)$. The pattern of abuse was episodic $(25 \%)$, recurrent $(33 \%)$, or chronic $(42 \%)$. $57 \%$ of the women saw a need to report their case as they felt nothing could be done about it. The study showed that though women were the victims of violence, they were surprisingly also the perpetrators and astute enforcers of the practice, as well as those 
who vehemently opposed any form of change. Women can play key and effective roles in eliminating gender-based violence and in initiating and implementing programmes that guarantee their reproductive and human rights. They should, therefore, be strengthened and encouraged to champion issues that affect their well-being.

17-02 Prevalence and Perceptions of Menopause in an Urban Community in Nigeria F. M. OlaOlorun; T. O. Lawoyin (University College Hospital, Ibadan, Nigeria) Background: Menopause represents the end of a womanýs reproductive years. A woman's attitudes to the changes that occur to her around this period vary widely and many misconceptions exist.

Objectives: To determine the prevalence of menopause and the perceptions of women in Ibadan, an urban community in Nigeria to menopause as well as to identify factors which affect this perception.

Methods: Community-based cross-sectional survey with both quantitative and qualitative components. A multistage sample was used to interview women aged 4060 years, irrespective of their menopausal status. Six focus group discussions including a total of 49 women were also conducted.

Results: A total of 1210 women were interviewed. Of these, $40.4 \%$ had attained menopause. Attitudes were generally good with a mean attitude score of 13.68 2.05 (maximum score: 20; good attitude score: 13-20). Following linear regression analysis, women with 1-4 children were more likely than women who were nulliparous or grandmultiparous to show a positive attitude to menopause (OR: 1.13; 95\% CI: 1.00-1.27).

Conclusion: The many misconceptions of menopause should be addressed using Behavior Change Communication methods in order to improve the quality of life of women in this study area through the menopausal transition and thereafter.

\section{7-03 Pilot Study on Depression among Secondary School Students from Urban and Semi Urban Schools in Selangor, Malaysia}

Adlina Narimah (UITM, SHAH ALAM)

Introduction: Depressive disorders occur in approximately $2 \%$ of primary schoolaged children and $4 \%$ to $8 \%$ of adolescents. Nearly half $(45 \%)$ of adolescents with major depression relapse in young adulthood. Children and adolescents with depression are also at increased risk for suicide, substance use disorders, early pregnancy, poor academic performance, and impaired psychosocial functioning. This study was conducted because there is insufficient data or information pertaining to the mental health of Malaysian children and there is virtually none to highlight the possible factors influencing it.

Objective: To study the prevalence and factors affecting depression among secondary school students.

Methodology: A sample of 2,048 students using stratified sampling from 2 urban schools (SMK Sri Permata and SMK Kelana Jaya, Petaling Jaya) and 3 semiurban schools (SMK Sungai Pelek Sepang, Sekolah SMK SriTanjung Kuala Selangor and SMK Sultan Abdul Aziz, Kuala Selangor) participated in the study. The instrument used was the validated Maria Kovacs Children's Depression Inventory for screening of depression.

Results:

- $10.3 \%$ of the students have some degree of depression.

- $9.2 \%$ have Negative Mood 
- $5 \%$ have interpersonal problems

- $19.7 \%$ suffer from ineffectiveness

- $9.8 \%$ have anhedonia

- $10.6 \%$ have poor self esteem

- The mean T-score of 54.73 among the females is significantly higher than the mean T-score of 51.92 among the males. This implies that the females are more prone to depression compared to the males.

- The Chinese students are more depressed compared to Indian students.

- Students whose parents had no formal education or had only primary education showed were more depressed than students whose parents had secondary, college or university education.

- Depression increased with increasing number of siblings

- Depressed students are at higher risk of developing habits of gum sniffing, drug abuse, alcohol abuse and stealing.

\section{7-04 Age at Menopause among Poor Urban Women}

\section{Matilda Saliba; Marwan Khawaja (American University of Beirut)}

This paper examines the timing of natural menopause and its correlates among poor urban residents. The study was based on a survey of ever married women residing in three urban Lebanese communities. The data used were taken from the reproductive health questionnaire of the Urban Health Study conducted by the Center of Research on Population and Health at the American University of Beirut. The sample size was 1050 women aged between 34 and 60 years old. Survival analysis was used to calculate the median age at menopause and to draw probability curves of remaining pre menopausal. Cox regression was applied to examine the factors associated with the onset of menopause. The median age at natural menopause was 50.8 years. Years of education was shown to be significantly related to later age at menopause while number of pregnancies was associated with an earlier age at menopause.

17-05 Social Support and Trust as Key Determinant of Condom Use in Rwanda: Evidence from the High Risk Youth Survey

Edouard Talnan (PSI); Staci Leuschner (PSI); Nicola Hobby (PSI) (Population Services International (PSI))

Background: Several studies (2005 DHS, 2005 Condom Accessibility Study, 2005 PLACE study) demonstrate that condom use remains low among sexually active youth. PSI/Rwanda conducted a quantitative study in August 2006 to identify determinants of condom use among youth who frequent bars in high transmission zones. This study focused on social support from relatives and trust in partners as key factors associated with condom use and assessed the effectiveness of communications activities.

Methods: The study was conducted in three urban areas of Rwanda; Kigali Ville, Gisenyi and Byumba. 2,961 surveys were completed among youth 15 to 29. Youth who reported having a regular, casual or paid partner within the last 12 months were included in the analysis. 839 cases were analyzed using multivariate logistical regression in SPSS.

Results: While knowledge about HIV/AIDS is high, non-users lack the confidence and self-efficacy to use condoms consistently. Condom use at last sex act was reported at $65 \%$; however consistent condom use was only $26 \%$. Segmentation analysis 
identified social support from partners and trusting partner as significant factors of condom use. Condom users were more likely than non-users to:

Feel social support from their partner about condom use;

Disagree with statements that their partner would lose trust in them if they asked him/her to use condoms

Believe their partner's past sexual behavior puts them more at risk for HIV/AIDS.

Conclusions: Programs should focus on increasing social support among young people to openly speak about condoms with friends and negotiate condom use with partners. Interpersonal communications campaigns should include condom demonstrations to increase self-efficacy for their use correctly. Messages should reinforce positive health behaviors such as consistent condom use regardless of partner type and disassociate trust from condoms.

\section{URBAN HEALTH POLICY}

\section{8-01 The Decline in Infant Mortality Medical Technology and Prenatal Care}

Sule Calikoglu, MA, MPP (Johns Hopkins Bloomberg School of Public Health)

Infant mortality is largely an urban phenomenon in the United States. This is especially frustrating because inner-city neighborhoods have stubbornly high rates despite the proximity of major health care institutions and several interventions specifically targeted on these areas.

This study attempts to contribute to the debate by analyzing the relationship between changes in infant mortality rates and trends in three sets of policy variables: prenatal care, maternal risk factors, and medical advancement. I compared two groups of cities in the US: those that met the Healthy People 2000 goals, and those that had not, specifically for both the neonatal mortality rate (NMR) and postneonatal mortality rate (PNMR), individually. My goal was to detect any important differences in trends of the three policy variables between cities that had achieved the Healthy People target rate and those that did not.

The results offer tentative support for two of the expected relationships between mortality rates and the three sets of policy variables.

First, cities with both NMR and PNMR below the target rates experienced a greater decline from 1990 to 2000 in the percent of women receiving late or no care than cities with rates above the targets.

Second, an increase in medical technology was associated with the decline in the NMR, although this relationship was less significant between 1990 and 2000.

Contrary to the NMR, medical technology did not show any significant relationship with the PNMR. Among maternal factors, only teenage childbearing was associated with a decline in the PNMR in the 1980ýs. However, the stronger decline in the PNMR occurred in the 1990s, which corresponds to the Sudden Infant Death Syndrome Campaign. Although this study is preliminary and explanatory, the research results suggest that infant mortality reduction policies need to address the NMR and PNMR individually and focus on the postneonatal period of fragile infants.

\section{8-02 Communication, Health \& Environment: Healthy Public Policy \& Technology at the Local Level}

Charles-Antoine Rouyer (Hygeia Healthy Communication saine Inc.)

Abstract (173 words): Access to modern information technologies enabled a community group downtown Toronto, Canada, to reframe the issue of the 
expansion of a cultural institution around the theme of urban health and quality of life, focusing on the green space, Grange Park, directly next to the new proposed building. Eventually, the community managed to bring the City government and the cultural institution to engage in a full community consultation process (bottom-up approach.)

The role of communication and information technology would then be presented in a broader theoretical model from a healthy public policy/urban health promotion perspective, within a systems thinking context.

The model presents the urban health process as a complex system of 16 different parameters and 216 combinations $(6 \times 4 \times 3 \times 3)$, including health, environment, economy, governance (private/public/community sectors), in a multidisciplinary and dynamic context, where information, public opinion, mass media and new narrowcasting media all interact and contribute to shape healthy public policies.

This paper would highlight several interactions between these 16 different factors relating to the main case studies and other specific local examples.

MODEL OVERVIEW:

Health: physical, mental, emotional, social, financial, spiritual (6)

Environment: natural, built, social, economic (4)

Communication: content, process, context (3)

Governance: public, private, civil society (3)

18-03 District of Columbia City-wide Patient Navigation Research Program (DC-PNRP) Steven R. Patierno, PhD, Principal Investigator; Nancy L.. LaVerda, MPH, Senior Research Associate (George Washington University Cancer Institute (GWCI))

Minority breast cancer mortality rates in Washington, DC are among the Nation's highest with a significant differential compared to whites. Evidence suggests the cause of this disparity is late stage at diagnosis and/or delayed initiation of treatment. DC-PNRP is one of 9 U.S. sites included in an NCI/ACS national research program evaluating whether or not using patient navigators (trained health workers assisting patients in reducing barriers to obtaining health care while addressing cultural/psychological factors) can reduce the breast cancer disparity in DC. Our hypotheses include: Patient navigation will be more effective than "usual care" in decreasing time from suspicious breast finding to diagnostic resolution and time from diagnosis to breast cancer treatment initiation. We expect to enroll 1400 women at the point of abnormal screen with minorities targeted at all 6 participating DC sites. The comparison groups include historical controls selected from records at the sites and concurrent controls selected from DC Department of Health records. The study is on a 5-year timeline with data collection beginning February 2007 and extending to 2010. A complex infrastructure was established with site-specific SOPs providing for common data collection and communication links to assure protocol adherence. An intricate national system was developed to assure agreement on data elements across sites.

Local area training was conducted to supplement National. A city-wide patient navigation program can be established among non-affiliated care centers to assure the underserved receive timely, high-quality care.

Existence of the well-established DC Cancer Consortium, plus the commitment of DC's major medical centers to reducing cancer disparities, suggests DC-PNRP will continue to be sustainable and successful after conclusion of the research study. 